\title{
ENUMERATION OF MEANDERS AND MASUR-VEECH VOLUMES
}

\author{
VINCENT DELECROIX ${ }^{1}$, ÉLISE GOUJARD ${ }^{2}$, PETER ZOGRAF $^{3}$ and \\ ANTON ZORICH ${ }^{4}$ \\ ${ }^{1}$ Laboratoire Bordelais de Recherche en Informatique, 33405 Talence, France; \\ email: vincent.delecroix@u-bordeaux.fr \\ ${ }^{2}$ Institut de Mathématiques de Bordeaux, 33405 Talence, France; \\ email: elise.goujard@gmail.com \\ ${ }^{3}$ Steklov Math. Institute and Chebyshev Laboratory, St. Petersburg 199178, Russia; \\ email: zograf@pdmi.ras.ru \\ ${ }^{4}$ Center for Advanced Studies, Skoltech, Institut Mathématique de Jussieu, 75205 Paris, France; \\ email: anton.zorich@gmail.com
}

Received 14 May 2019; accepted 23 December 2019

\begin{abstract}
A meander is a topological configuration of a line and a simple closed curve in the plane (or a pair of simple closed curves on the 2-sphere) intersecting transversally. Meanders can be traced back to H. Poincaré and naturally appear in various areas of mathematics, theoretical physics and computational biology (in particular, they provide a model of polymer folding). Enumeration of meanders is an important open problem. The number of meanders with $2 N$ crossings grows exponentially when $N$ grows, but the long-standing problem on the precise asymptotics is still out of reach.

We show that the situation becomes more tractable if one additionally fixes the topological type (or the total number of minimal arcs) of a meander. Then we are able to derive simple asymptotic formulas for the numbers of meanders as $N$ tends to infinity. We also compute the asymptotic probability of getting a simple closed curve on a sphere by identifying the endpoints of two arc systems (one on each of the two hemispheres) along the common equator.

The new tools we bring to bear are based on interpretation of meanders as square-tiled surfaces with one horizontal and one vertical cylinder. The proofs combine recent results on Masur-Veech volumes of moduli spaces of meromorphic quadratic differentials in genus zero with our new observation that horizontal and vertical separatrix diagrams of integer quadratic differentials are asymptotically uncorrelated. The additional combinatorial constraints we impose in this article yield explicit polynomial asymptotics.

2010 Mathematics Subject Classification: 32G15 (primary); 57M50, 30F30, 05C30 (secondary)

(c) The Author(s) 2020. This is an Open Access article, distributed under the terms of the Creative Commons AttributionNonCommercial-ShareAlike licence (http://creativecommons.org/licenses/by-nc-sa/4.0/), which permits non-commercial re-use, distribution, and reproduction in any medium, provided the same Creative Commons licence is included and the original work is properly cited. The written permission of Cambridge University Press must be obtained for commercial re-use.
\end{abstract}




\section{Introduction}

In the seminal paper [Mi], Mirzakhani computed the asymptotics for the number of simple closed geodesics on a hyperbolic surface of constant negative curvature. In particular, she proved that asymptotically, when the bound for the length of simple closed geodesics tends to infinity, the probability of getting a separating or nonseparating geodesic becomes independent of the hyperbolic metric.

We count the asymptotics for the number of pairs of transverse simple closed curves, or meanders, of a fixed combinatorial type on a sphere when the number of intersections tends to infinity. Our starting observation is that a pair of transverse simple closed curves which is seemingly a purely combinatorial object defines a natural complex structure and an 'integer' meromorphic quadratic differential on the original sphere.

Mirzakhani established a relation between the counting of simple closed curves and Weil-Petersson volumes of the moduli spaces of bordered hyperbolic surfaces; see [Mi, Theorem 5.3]. Counting pairs of transverse simple closed curves leads naturally to Masur-Veech volumes of the moduli spaces of meromorphic quadratic differentials with at most simple poles. In both situation, an essential ingredient is the ergodicity of a certain group action. In Mirzakhani's case, it is the action of the mapping class group $\operatorname{Mod}_{g, n}$ on the space of measured laminations $\mathcal{M} \mathcal{L}_{g, n}$. In our setting, it will be the $\mathrm{GL}_{2}(\mathbb{R})$-action on (strata of) the moduli space of quadratic differentials $\mathcal{Q}(\xi)$, where $\xi$ denotes the number of simple poles, the number of zeros and the degrees of zeros of meromorphic quadratic differentials in the stratum $\mathcal{Q}(\xi)$. Both moduli spaces have integral piecewise linear structures and both counting problems (count of multicurves and count of pairs of transverse multicurves) can be formulated in terms of counting integer points in, respectively, $\mathcal{M} \mathcal{L}_{g, n}$ and $\mathcal{Q}(\xi)$. A simple example is provided by the set of primitive integer points in $\mathbb{Z}^{2} \subset \mathbb{R}^{2}$. They have asymptotic density $\delta=\frac{6}{\pi^{2}}$, meaning that in a ball of radius $R$ centered around the origin, there are $\delta \pi R^{2}+o\left(R^{2}\right)$ such primitive points. Moreover, the density is uniform (see Section 4.4 for formal definitions).

The other essential ingredient of our proof that has no equivalent in Mirzakhani setting is a noncorrelation result that we deduce from the product structure on the strata of quadratic differentials. More precisely, we prove that for suitable subsets $\mathcal{D}_{1}$ and $\mathcal{D}_{2}$ of square-tiled surfaces, the density $\delta\left(\mathcal{D}_{1} \cap \mathcal{D}_{2}\right)$ of their intersection is equal to the product of densities $\delta\left(\mathcal{D}_{1}\right) \cdot \delta\left(\mathcal{D}_{2}\right)$ (see Theorem 4.16). This noncorrelation result is visible in our meander count: the constant $\operatorname{cyl}_{1,1}\left(\mathcal{Q}\left(1^{s},-1^{s+4}\right)\right)$ appearing in Theorem 1.1 has an explicit product type expression given in (2.9). 


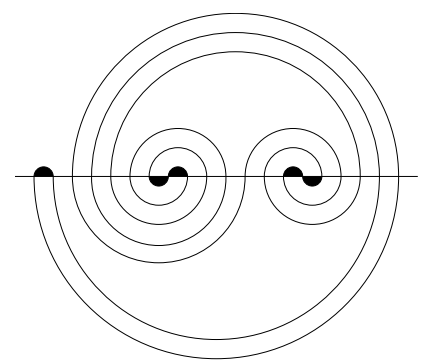

Contributes to $\mathcal{M}_{5}^{+}(N)$

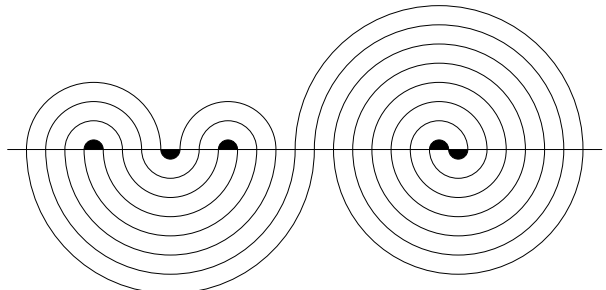

Contributes to $\mathcal{M}_{5}^{-}(N)$

Figure 1. Meander with a maximal arc ('rainbow') on the left and without one on the right. Both meanders have five minimal arcs ('pimples').

1.1. Structure of the paper. In the first section, we state our results on meander enumeration. The link with quadratic differentials and Masur-Veech volumes is explained in Section 2. Section 3 provides the proof of our results on meander count; it uses general results from the subsequent Section 4. The last section of the article proves the equidistribution and noncorrelation results in the moduli space of quadratic differentials.

The paper is organized in such a way that Section 4 can be omitted by the readers interested only in meanders. On the other hand, the readers interested only in square-tiled surfaces and moduli spaces of quadratic differentials can pass directly to Section 4.

Finally, Appendix A describes the geometry underlying two natural normalizations of the Masur-Veech volume element on the moduli spaces of quadratic differentials. This clarification is needed to apply the results from Section 4 to meander count.

\subsection{Counting meanders with given number of minimal arcs. A closed} plane meander is a smooth closed curve in the plane transversally intersecting the horizontal line as in Figure 1. According to the paper [LdZv] of Lando and Zvonkine (serving as a reference paper in the literature on meanders), the notion 'meander' was suggested by Arnold in [Arn] though meanders were studied already by Poincaré [Po]. Meanders appear in various contexts, in particular, in physics; see [DiFGG1]. The number of meanders with $2 N$ crossings is conjecturally asymptotic to const $\cdot R^{2 N} \cdot N^{\alpha}$, where $R^{2} \approx 12.26$ and $\alpha \approx-3.42$ are constants (we refer to [Jen] for the values of the constants). The conjectural 
exact value $\alpha=-\frac{29+\sqrt{145}}{12}$ is given in [DiFGG2]. They derived this value from a related conformal field theory.

A meander has a maximal arc (a 'rainbow' in terminology of [ACPRS]) if it has an arc joining the leftmost and the rightmost crossings with the horizontal line. Otherwise, the meander does not have a maximal arc. The meander on the left of Figure 1 has a maximal arc, while the one on the right does not.

By minimal arc ('pimple' in terminology of [ACPRS] or 'internal arch' in terminology of [DiFGG1]), we call an arc which does not have any crossings inside. The areas between the horizontal line and the minimal arcs of meanders are colored in black in Figure 1; each of the two meanders has $p=5$ minimal arcs.

By convention, in this paper, we do not consider the trivial meander represented by a circle. All other meanders satisfy $p \geqslant 3$ when they have a maximal arc and $p \geqslant 4$ when they do not.

Let $\mathcal{M}_{p}^{+}(N)$ and $\mathcal{M}_{p}^{-}(N)$ be the numbers of meanders, respectively, with and without maximal arc ('rainbow') and having at most $2 N$ crossings with the horizontal line and exactly $p$ minimal arcs ('pimples'). We consider $p$ as a parameter and we study the leading terms of the asymptotics of $\mathcal{M}_{p}^{+}(N)$ and $\mathcal{M}_{p}^{-}(N)$ as $N \rightarrow+\infty$.

THEOREM 1.1. For any fixed $p$, the numbers $\mathcal{M}_{p}^{+}(N)$ and $\mathcal{M}_{p}^{-}(N)$ of meanders with $p$ minimal arcs (pimples) and with at most $2 N$ crossings have the following asymptotics as $N \rightarrow+\infty$ :

$$
\begin{aligned}
\mathcal{M}_{p}^{+}(N) & =2(p+1) \cdot \frac{\operatorname{cyl}_{1,1}\left(\mathcal{Q}\left(1^{p-3},-1^{p+1}\right)\right)}{(p+1) !(p-3) !} \cdot \frac{N^{2 p-4}}{4 p-8}+o\left(N^{2 p-4}\right) \\
& =\frac{2}{p !(p-3) !}\left(\frac{2}{\pi^{2}}\right)^{p-2} \cdot\left(\begin{array}{c}
2 p-2 \\
p-1
\end{array}\right)^{2} \cdot \frac{N^{2 p-4}}{4 p-8}+o\left(N^{2 p-4}\right) . \\
\mathcal{M}_{p}^{-}(N) & =\frac{2 \operatorname{cyl}_{1,1}\left(\mathcal{Q}\left(1^{p-4}, 0,-1^{p}\right)\right)}{p !(p-4) !} \cdot \frac{N^{2 p-5}}{4 p-10}+o\left(N^{2 p-5}\right) \\
& =\frac{4}{p !(p-4) !}\left(\frac{2}{\pi^{2}}\right)^{p-3} \cdot\left(\begin{array}{c}
2 p-4 \\
p-2
\end{array}\right)^{2} \cdot \frac{N^{2 p-5}}{4 p-10}+o\left(N^{2 p-5}\right) .
\end{aligned}
$$

The quantities $\operatorname{cyl}_{1,1}\left(\mathcal{Q}\left(1^{p-3},-1^{p+1}\right)\right)$ and $\operatorname{cyl}_{1,1}\left(\mathcal{Q}\left(1^{p-4}, 0,-1^{p}\right)\right)$ in the above formulas are related to Masur-Veech volumes of the moduli space of meromorphic quadratic differentials. Their definition and role are discussed in Section 2. Theorem 1.1 is proved in Section 3.5 with the exception of the explicit expressions for these two quantities evaluated in Corollary 4.30 in Section 4.10. 
Note that the number $\mathcal{M}_{p}^{+}(N)$ grows as $N^{2 p-4}$, while $\mathcal{M}_{p}^{-}(N)$ grows as $N^{2 p-5}$. This means that for large $N$, all but a negligible fraction of meanders having any given number $p$ of minimal arcs (pimples) do have a maximal arc (rainbow) as the left one in Figure 1.

Our approach to counting meanders differs from the traditional one: we fix the combinatorics of the meander and then count the asymptotic number of meanders of chosen combinatorial type as the number of intersections $N$ tends to infinity. Our settings can be seen as a zero temperature limit in the thermodynamical sense, where the complexity of a meander is measured in terms of the number of minimal arcs. Namely, let us count meanders with the weight $e^{-\beta p}$, where $\beta>0$ is a parameter and $p$ is the number of minimal arcs. Then $\beta=0$ corresponds to the standard count of meanders. In the 'zero temperature limit' $\beta \rightarrow+\infty$ meanders with few minimal arcs, as considered in this paper, become predominant.

Applying Stirling's formula, we get the following asymptotics for the coefficients in formulas (1.1) and (1.2) for large values of parameter $p$ :

$$
\begin{gathered}
\frac{2}{p !(p-3) !}\left(\frac{2}{\pi^{2}}\right)^{p-2} \cdot\left(\begin{array}{c}
2 p-2 \\
p-1
\end{array}\right)^{2} \cdot \frac{1}{4 p-8} \sim \frac{\pi^{2}}{256} \cdot\left(\frac{32 e^{2}}{\pi^{2} p^{2}}\right)^{p} \\
\text { for } p \gg 1 . \\
\frac{4}{p !(p-4) !}\left(\frac{2}{\pi^{2}}\right)^{p-3} \cdot\left(\begin{array}{c}
2 p-4 \\
p-2
\end{array}\right)^{2} \cdot \frac{1}{4 p-10} \sim \frac{\pi^{2} e^{2}}{128 p} \cdot\left(\frac{32 e^{2}}{\pi^{2} p^{2}}\right)^{p-1} \\
\text { for } p \gg 1
\end{gathered}
$$

(we again recall that in our setting, we always assume that $N \gg p$ ).

In Section 3.5, we provide an analogous statement, Theorem 3.8, which counts meanders in the setting where the combinatorial type is specified in a more detailed way.

1.3. Counting meanders with given reduced arc systems. Extending the horizontal segment of a plane meander to the infinite line and passing to a one-point compactification of the plane, we get a meander on the 2-sphere. A meander on the sphere is a pair of transversally intersecting labeled simple closed curves. It will be always clear from the context whether we consider meanders in the plane or on the sphere. Essentially, we adhere to the following dichotomy: enumerating meanders, as in the previous section, we work with meanders in the plane, while considering frequencies of pairs of simple closed curves among 

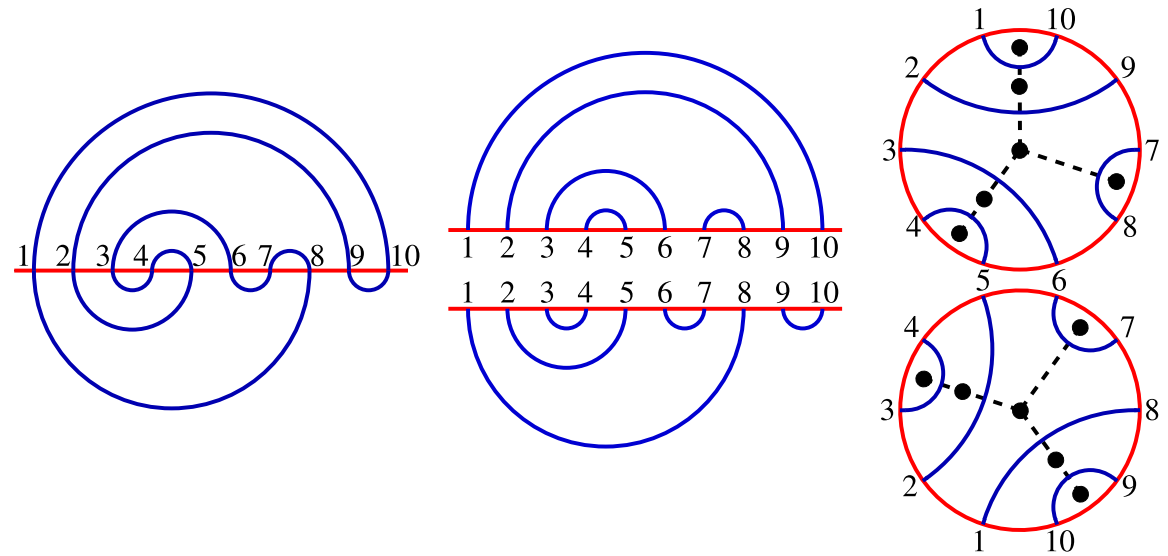

Figure 2. A meander on the left. The associated pair of arc systems in the middle. The same arc systems on the discs and the associated dual trees on the right. Both arch systems on the discs have a tripod $\zeta$ as a reduced dual tree.

more complicated pairs of multicurves, as in the current section, we work with meanders on the sphere.

Each meander defines a pair of arc systems in discs as in Figure 2. Arcs in each of the two arc systems do not intersect pairwise. An arc system on the disc (also known as a 'chord diagram') can be encoded by the dual tree; see the trees in dashed lines on the right pictures in Figure 2. Namely, the vertices of the tree correspond to the faces in which the arc system cuts the disc; two vertices are joined by an edge if and only if the corresponding faces have a common arc. It is convenient to simplify the dual tree by forgetting all vertices of valence 2 . We call the resulting tree the reduced dual tree.

It is much easier to count arc systems (for example, arc systems sharing the same reduced dual tree). However, this does not simplify the meander count since identifying a pair of arc systems with the same number of arcs by the common equator, we sometimes get a meander and sometimes a curve with several connected components; see Figure 3.

We now consider the more specialized setting where we fix a pair of plane trees and count meanders whose corresponding pair of arc systems have these given dual trees. Let us mention that everywhere in this paper, we consider only plane trees, that is, trees embedded into the plane.

Let $\left(\mathcal{T}_{\text {top }}, \mathcal{T}_{\text {bottom }}\right)$ be a pair of plane trees with no vertices of valence 2 . We consider the arc system with the same number of $\operatorname{arcs} n \leqslant N$ on a labeled pair of oriented discs having $\mathcal{T}_{\text {top }}$ and $\mathcal{T}_{\text {bottom }}$ as reduced dual trees. We draw the arc 

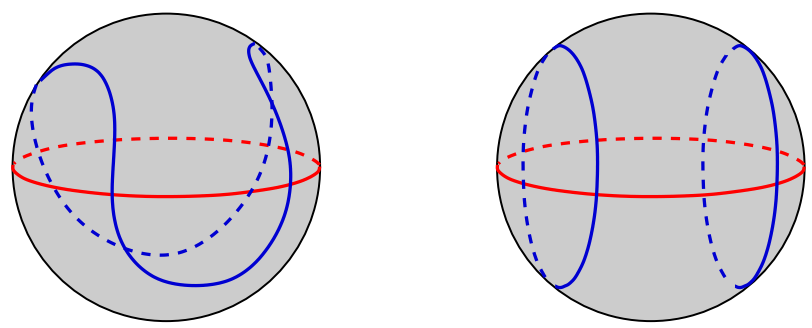

Figure 3. Gluing two hemispheres with arc systems along the common equator, we may get either a single simple closed curve (as on the left picture) or a multicurve with several connected components (as on the right picture).

system corresponding to $\mathcal{T}_{\text {top }}$ on the northern hemisphere and the arc system corresponding to $\mathcal{T}_{\text {bottom }}$ on the southern hemisphere. There are $2 n$ ways (up to isotopy) to identify the boundaries of two hemispheres into the sphere in such a way that the endpoints of the arcs match. We consider all possible triples

( $n$-arc system of type $\mathcal{T}_{\text {top }} ; n$-arc system of type $\mathcal{T}_{\text {bottom }} ;$ identification) as described above for all $n \leqslant N$. Define

$$
\mathrm{P}_{\text {connected }}\left(\mathcal{T}_{\text {top }}, \mathcal{T}_{\text {bottom }} ; N\right):=\frac{\text { number of triples giving rise to meanders }}{\text { total number of different triples }} .
$$

THEOREM 1.2. For any pair of trivalent plane trees $\mathcal{T}_{\text {bottom }}, \mathcal{T}_{\text {top }}$, having the total number $p$ of leaves (vertices of valence 1), the following limit exists:

$$
\begin{aligned}
\lim _{N \rightarrow+\infty} \mathrm{P}_{\text {connected }}\left(\mathcal{T}_{\text {bottom }}, \mathcal{T}_{\text {top }} ; N\right)=\mathrm{P}_{1}\left(\mathcal{Q}\left(1^{p-4},-1^{p}\right)\right) \\
=\frac{\operatorname{cyl}_{1}\left(\mathcal{Q}\left(1^{p-4},-1^{p}\right)\right)}{\operatorname{Vol}_{1}\left(\mathcal{Q}_{1}\left(1^{p-4},-1^{p}\right)\right)}=\frac{1}{2}\left(\frac{2}{\pi^{2}}\right)^{p-3} \cdot\left(\begin{array}{c}
2 p-4 \\
p-2
\end{array}\right) .
\end{aligned}
$$

The quantity $\operatorname{cyl}_{1}\left(\mathcal{Q}\left(1^{p-4},-1^{p}\right)\right)$ in the above formula is related to MasurVeech volume of the moduli space of meromorphic quadratic differentials. Its definition and role will be discussed in Section 2.

The quantity $\mathrm{P}_{1}\left(\mathcal{Q}\left(1^{p-4},-1^{p}\right)\right)$ can be seen as the asymptotic probability that a random gluing of a pair of random arc systems with $p$ minimal arcs produces a meander. To be more accurate, one should rather speak of asymptotic density of meanders among the resulting multicurves.

Theorem 1.2 is proved at the end of Section 3.4. We will actually state and prove a more general statement, Theorem 3.7, where not only trivalent trees are considered. 
The fact that this asymptotic density is nonzero is already somehow unexpected. For example, for the pair of trees as on the right side of Figure 2, each of the reduced trees contains a single vertex of valence 3 and three vertices of valence 1 (three leaves), so we have six leaves in total. The corresponding asymptotic density for such a pair of reduced trees is equal to

$$
\mathrm{P}_{\text {connected }}(\dot{\varkappa}, \because)=\frac{280}{\pi^{6}} \approx 0.291245,
$$

which is not even close to 0 .

Stirling's formula gives the following asymptotics for $\mathrm{P}_{1}\left(\mathcal{Q}\left(1^{p-4},-1^{p}\right)\right)$ for large values of parameter $p$ :

$$
\mathrm{P}_{1}\left(\mathcal{Q}\left(1^{p-4},-1^{p}\right)\right)=\frac{1}{2}\left(\frac{2}{\pi^{2}}\right)^{p-3} \cdot\left(\begin{array}{c}
2 p-4 \\
p-2
\end{array}\right) \sim \frac{2}{\sqrt{\pi p}} \cdot\left(\frac{8}{\pi^{2}}\right)^{p-3} \text { for } p \gg 1
$$

(we recall that in our setting, we always assume that $N \gg p$ ).

Another unexpected fact that follows from Theorem 1.2 is that the way the leaves (univalent vertices) are distributed between the two trees is irrelevant: the answer depends only on the total number $p$ of leaves. This observation suggests an alternative (and much less restrictive) way to fix combinatorics of the meanders. Namely, we can fix only the total number $p$ of leaves (vertices of valence 1 ) of the two trees together, where $p \geqslant 4$.

THEOREM 1.3. Let $p \geqslant 4$. The density $\mathrm{P}_{\text {connected }}(p ; N)$ of meanders obtained by all possible identifications of all arc systems with at most $N$ arcs represented by all possible pairs of (not necessarily trivalent) plane trees having the total number $p$ of leaves (vertices of valence 1$)$ has the same limit $\mathrm{P}_{1}\left(\mathcal{Q}\left(1^{p-4}\right.\right.$, $\left.-1^{p}\right)$ ) as the density $\mathrm{P}_{\text {connected }}\left(\mathcal{T}_{\text {bottom }}, \mathcal{T}_{\text {top }} ; N\right)$ of meanders represented by any individual pair of trivalent plane trees with the total number $p$ of leaves:

$$
\begin{aligned}
\lim _{N \rightarrow+\infty} \mathrm{P}_{\text {connected }}(p ; N)=\mathrm{P}_{1}\left(\mathcal{Q}\left(1^{p-4},-1^{p}\right)\right) \\
=\frac{\operatorname{cyl}_{1}\left(\mathcal{Q}\left(1^{p-4},-1^{p}\right)\right)}{\operatorname{Vol}_{1} \mathcal{Q}_{1}\left(1^{p-4},-1^{p}\right)}=\frac{1}{2}\left(\frac{2}{\pi^{2}}\right)^{p-3} \cdot\left(\begin{array}{c}
2 p-4 \\
p-2
\end{array}\right) .
\end{aligned}
$$

The same statement with the same limit is valid if we consider pairs of plane trees having at most $p$ leaves for the two trees together instead of exactly $p$ leaves.

Theorem 1.3 is proved at the end of Section 3. The proof is based on the fact that the contribution of any pair of trees where at least one of the trees has a vertex of valence 4 or higher is negligible in comparison with the contribution of any pair of trivalent trees. 
REMARK 1.4. All the results of Section 1 concerning square-tiled surfaces in strata of quadratic differentials of genus 0 generalize to higher genera and even to the general situation of arithmetic invariant suborbifolds (see Section 4.1). In this latter setting, some subtle finiteness issues arise and will be treated elsewhere to avoid overloading the current paper.

\section{Outline of counting technique}

2.1. Pairs of transverse multicurves on the sphere as square-tiled surfaces. A multicurve on the sphere is a collection of pairwise nonintersecting smooth simple closed curves. Two multicurves are considered equivalent if one is the image of the other by a homeomorphism. For each positive integer $n$, the number of equivalence classes of multicurves made of $n$ components is finite.

DEFINITION 2.1. We say that two multicurves on the sphere form a transverse connected pair if any intersection between any connected component of the first curve and any connected component of the second curve is transverse and if in addition the union of the two multicurves is connected.

Having a transverse connected pair of multicurves, we always assume that the pair is ordered. By convention, the first multicurve is called 'horizontal' and the second one 'vertical'. We consider natural equivalence classes of transverse connected pairs of multicurves up to diffeomorphisms preserving the orientation of the sphere and respecting horizontal and vertical labeling.

Let $\mathcal{G}$ be the graph defined by a transverse connected pair of multicurves. The vertices of $\mathcal{G}$ are intersections of the multicurves, so all vertices of $\mathcal{G}$ have valence 4. Hence, all faces of the dual graph $\mathcal{G}^{*}$ are 4-gons. The edges of $\mathcal{G}^{*}$ dual to horizontal edges of $\mathcal{G}$ will be called vertical and those dual to the vertical edges of $\mathcal{G}$ will be called horizontal. By construction, any two nonadjacent edges of any face of $\mathcal{G}^{*}$ are either both horizontal or both vertical.

Choosing identical metric squares as faces of $\mathcal{G}^{*}$, we get a square-tiled surface. We have proved the following statement.

PROPOSITION 2.2. There is a natural one-to-one correspondence between transverse connected pairs of multicurves on the sphere and square-tiled surfaces of genus 0 , where the square tiling is given by the dual graph of the graph formed by the union of two multicurves.

A square-tiled surface defines a meromorphic quadratic differential $q$ having the form $(d z)^{2}$ in the natural coordinate on each square. Simple poles of $q$ correspond to bigons of $\mathcal{G}$; zeros of order $j \in \mathbb{N}$ correspond to $(2 j+4)$-gons. 

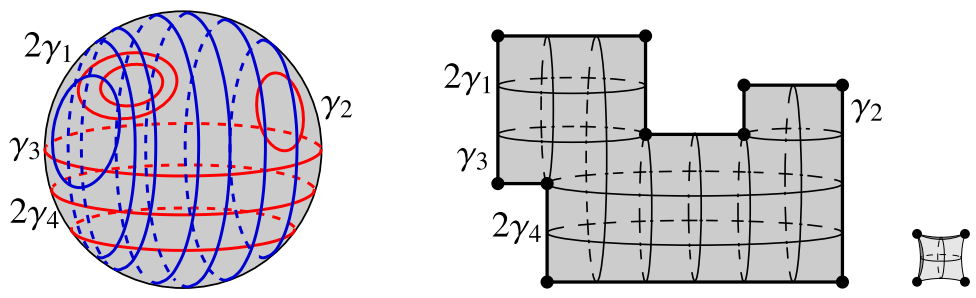

Figure 4. The dual graph to a transverse connected pair of multicurves on a sphere defines a square-tiled surface. In this particular example, the horizontal multicurve has the form $2 \gamma_{1}+\gamma_{2}+\gamma_{3}+2 \gamma_{4}$; its primitive components $\gamma_{1}, \ldots$, $\gamma_{4}$ represent the four maximal horizontal cylinders; the weights represent the numbers of horizontal bands of squares in the corresponding cylinders.

REMARK 2.3. Note that speaking of a 'multicurve', one usually assumes that the components of a multicurve are neither contractible nor peripheral (that is, not freely homotopic to a boundary component). Given a transverse connected pair of multicurves in the sense of Definition 2.1 make a single puncture at every bigon, 6-gon, 8-gon and so on (equivalently, puncture every conical singularity of the associated square-tiled surface). By construction, each component of the horizontal (respectively vertical) multicurve is neither contractible nor peripheral on the resulting punctured surface, so we get a multicurve in the usual sense.

Traditionally, one represents a multicurve as a weighted sum $\gamma=h_{1} \gamma_{1}+\cdots+$ $h_{m} \gamma_{m}$ of the primitive components $\gamma_{1}, \ldots, \gamma_{m}$, which are already not pairwise freely homotopic on the punctured surface, and where the positive integer weight $h_{i}$ encodes the number of components of the multicurve $\gamma$ freely homotopic to the primitive component $\gamma_{i}$ for $i=1, \ldots, m$. In our case the primitive components $\gamma_{i}$ and the weights $h_{i}$ have particularly transparent interpretation in terms of geometry of the associated square-tiled surface. Namely, the primitive components $\gamma_{i}$ of the horizontal (respectively vertical) multicurve are in the natural correspondence with the maximal horizontal (respectively vertical) cylinders, while the associated weights $h_{i}$ represent the numbers of horizontal (respectively vertical) bands of squares in the corresponding cylinders.

The number of components of a multicurve $\gamma=h_{1} \gamma_{1}+\cdots+h_{m} \gamma_{m}$ is given by the sum $h_{1}+\cdots+h_{m}$ of the weights; the number $m$ represents the number of primitive components. The number of primitive components of the horizontal multicurve corresponds to the number of maximal horizontal cylinders of the associated square-tiled surface, while the number of components represents the total number of horizontal bands of squares. The square-tiled surface in Figure 4 has six horizontal bands of squares organized into four maximal flat cylinders 
filled with parallel horizontal closed regular geodesics. These six horizontal bands of squares correspond to six components of the associated horizontal multicurve $2 \gamma_{1}+\gamma_{2}+\gamma_{3}+2 \gamma_{4}$. The same square-tiled surface has seven vertical bands of squares organized into four maximal flat cylinders filled with parallel vertical closed regular geodesics. The associated vertical multicurve has seven components.

2.2. Counting square-tiled surfaces. The moduli space of meromorphic quadratic differentials on $\mathbb{C P} P^{1}$ with exactly $p$ simple poles is naturally stratified by the strata $\mathcal{Q}\left(v,-1^{|v|+4}\right)$ of quadratic differentials with prescribed orders of zeros ( $v_{i}$ zeros of order $i$ ) and with $p=|v|+4$ simple poles (see, for example, [Zor1] for references). Here

$$
|v|=1 \cdot v_{1}+2 \cdot v_{2}+3 \cdot v_{3}+\cdots .
$$

Under the above interpretation, transverse connected pairs of multicurves having fixed number of bigonal faces correspond to square-tiled surfaces with fixed number of simple poles. The transverse connected pairs of multicurves having fixed number $v_{1}$ of hexagonal faces, fixed number $v_{2}$ of octagonal faces and fixed number $v_{j}$ of $2(j+2)$-gonal faces for $j \in \mathbb{N}$ correspond to square-tiled surfaces in the fixed stratum $\mathcal{Q}\left(v,-1^{|v|+4}\right)$. In particular, the number of bigonal faces equals $|v|+4$. The number of squares in the square-tiled surface is the total number of crossings between the two multicurves.

Gluing two hemispheres with arc systems along the common equator as in Section 3.5, we get a transverse connected pair of multicurves. The horizontal multicurve has a single connected component, which is a simple closed curve represented by the equator, whereas the vertical multicurve may have several connected components. Such transverse connected pairs of multicurves correspond to square-tiled surfaces having a single horizontal band of squares.

Labeled connected pairs of transverse simple closed curves correspond to square-tiled surfaces having a single horizontal band of squares and a single vertical band of squares. Closed meanders in the plane correspond to squaretiled surfaces as above with a marked vertical side of one of the squares of the tiling.

Having translated our counting problems into the language of square-tiled surfaces, we are ready to present our approach in detail.

Square-tiled surfaces of fixed combinatorial type and Masur-Veech volumes. Any (generalized) partition $v=\left[0^{v_{0}} 1^{v_{1}} 2^{v_{2}} \ldots\right]$ determines a complex orbifold $\mathcal{Q}\left(v,-1^{|v|+4}\right)$ called a stratum of meromorphic quadratic differentials. The points of $\mathcal{Q}\left(v,-1^{|v|+4}\right)$ are the equivalence classes of meromorphic 
quadratic differentials with only simple poles on the sphere having $v_{0}$ marked points, $v_{1}$ zeros of order $1, v_{2}$ zeros of order 2, and so on. Each stratum $\mathcal{Q}(v$, $-1^{|v|+4}$ ) has an integral linear structure and hence a well-defined measure that is called the Masur-Veech measure. We denote by $\operatorname{Vol}_{1} \mathcal{Q}_{1}\left(\nu,-1^{|v|+4}\right)$ the Masur-Veech volume of the 'unit hyperboloid' in the stratum $\mathcal{Q}\left(v,-1^{|v|+4}\right)$. We refer the reader to Section 4.1 for precise definitions. By [AEZ2], the following formula holds:

$$
\mathrm{Vol}_{1} \mathcal{Q}_{1}\left(\nu,-1^{|v|+4}\right)=2 \pi^{2} \cdot(f(0))^{\nu_{0}}(f(1))^{\nu_{1}}(f(2))^{\nu_{2}} \cdots,
$$

where $|v|=1 \cdot v_{1}+2 \cdot v_{2}+\cdots$ and

$$
f(j)=\frac{j ! !}{(j+1) ! !} \cdot \pi^{j} \cdot \begin{cases}\pi & \text { if } j \text { is odd } \\ 2 & \text { if } j \text { is even }\end{cases}
$$

(here we use the notation

$$
j ! !:= \begin{cases}1 \cdot 3 \cdot 5 \cdots j & \text { when } j \text { is odd, } \\ 2 \cdot 4 \cdot 6 \cdots j & \text { when } j \text { is even, }\end{cases}
$$

and the common convention $0 ! !:=1$ ). This formula was originally conjectured by Kontsevich and recently proved in [AEZ2]. In this setting, zeros and poles of quadratic differentials are labeled, so the Masur-Veech volume of the stratum with unlabeled zeros and poles is smaller by the factor $(|v|+4) ! v_{0} ! v_{1} ! \cdots$. It follows from the definition of the Masur-Veech volume that the number $\mathcal{S}_{v}^{\text {labeled }}(N)$ of square-tiled surfaces (that is, the integral points in $\mathcal{Q}\left(v,-1^{|v|+4}\right)$; see Section 4.3 for the definition) in the stratum $\mathcal{Q}\left(v,-1^{|v|+4}\right)$ tiled with at most $2 N$ squares has asymptotics

$$
\mathcal{S}_{v}^{\text {labeled }}(N)=\operatorname{Vol}_{1} \mathcal{Q}_{1}\left(\nu,-1^{|v|+4}\right) \cdot \frac{N^{d}}{2 d}+o\left(N^{d}\right) \text { as } N \rightarrow+\infty,
$$

where

$$
d=\operatorname{dim}_{\mathbb{C}} \mathcal{Q}\left(v,-1^{|v|+4}\right)=\ell(v)+|v|+2
$$

and

$$
\ell(v):=v_{0}+v_{1}+\cdots .
$$

In Theorems 1.1 and 1.3, the number $p$ of bigons serves as 'combinatorial type' of a square-tiled surface. In this setting, formulas (2.3) and (2.4) imply that all but a negligible proportion of transverse connected pairs of multicurves having large number $N$ of intersections have only bigons, squares and hexagons as faces and correspond to square-tiled surfaces in the principal stratum $\mathcal{Q}\left(1^{p-4},-1^{p}\right)$. 
As an alternative choice of 'combinatorial type' of a square-tiled surface, one can specify the number of hexagons, octagons and so on separately, thus fixing the stratum $\mathcal{Q}\left(v,-1^{|v|+4}\right)$. This corresponds to the setting of Theorems 3.7 and 3.8. Under either choice, we have a simple asymptotic formula for the number of transverse connected pairs of multicurves of fixed combinatorial type with at most $2 \mathrm{~N}$ intersections.

REMARK 2.4 (Labeled versus nonlabeled zeros and poles). When we introduced square-tiled surfaces in Section 2.1 and identified them with transverse connected pairs of multicurves in Proposition 2.2, we did not label zeros and poles of the corresponding quadratic differential, which was quite natural in this setting. Traditionally, one labels zeros and poles of a square-tiled surface in the context of Masur-Veech volumes, so we usually do label zeros and poles. We recall the setting every time when there may be any ambiguity. The unlabeled and labeled count of square-tiled surfaces in the stratum $\mathcal{Q}\left(\nu,-1^{|v|+4}\right)$ differ by the integral factor $(|v|+4) ! v_{0} ! v_{1} ! \cdots$.

Square-tiled surfaces with fixed combinatorics. By Proposition 2.2, a squaretiled surface has exactly $k$ horizontal (respectively vertical) bands of squares if and only if the associated horizontal (respectively vertical) multicurve has exactly $k$ connected components; see Remark 2.3 at the end of Section 2.1. Counting square-tiled surfaces with exactly $k$ horizontal bands of squares admits an efficient combinatorial approach. In the case of a single horizontal band of squares corresponding to $k=1$, this count was performed in [DGZZ]. In Section 4.10, we reproduce the relevant computations that become particularly explicit in the case of the sphere.

THEOREM 2.5. Let $k \geqslant 1$ and let $v=\left[0^{v_{0}} 1^{v_{1}} 2^{v_{2}} \ldots\right]$ be a generalized partition. The number $\mathcal{S}_{k, v}^{\text {labeled }}(N)$ of square-tiled surfaces in the stratum $\mathcal{Q}\left(v,-1^{|v|+4}\right)$ with labeled zeros and poles tiled with at most $2 N$ squares organized into $k$ horizontal bands (that is, having the associated horizontal multicurve composed of $k$ connected components) has asymptotics

$$
\mathcal{S}_{k, v}^{\text {labeled }}(N)=\operatorname{cyl}_{k}\left(\mathcal{Q}\left(\nu,-1^{|v|+4}\right)\right) \cdot \frac{N^{d}}{2 d}+O\left(N^{d-1}\right) \quad \text { as } N \rightarrow+\infty,
$$

where the coefficient $\operatorname{cyl}_{k}\left(\mathcal{Q}\left(v,-1^{|v|+4}\right)\right)$ is a positive rational number. Moreover, in the case $k=1$, one has the following explicit expression:

$$
\operatorname{cyl}_{1}\left(\mathcal{Q}\left(\nu,-1^{|\nu|+4}\right)\right)=2 \cdot \sum_{\iota_{0}=0}^{\nu_{0}} \sum_{\iota_{1}=0}^{\nu_{1}} \sum_{\iota_{2}=0}^{\nu_{2}} \sum_{\ldots}^{\ldots}\left(\begin{array}{l}
\nu_{0} \\
\iota_{0}
\end{array}\right)\left(\begin{array}{c}
\nu_{1} \\
\iota_{1}
\end{array}\right)\left(\begin{array}{c}
\nu_{2} \\
\iota_{2}
\end{array}\right) \cdots\left(\begin{array}{c}
|\nu|+4 \\
|\iota|+2
\end{array}\right) .
$$


Here $\iota=\left[0^{\iota_{0}} 1^{\iota_{1}} 2^{\iota_{2}} \ldots\right], d=\operatorname{dim}_{\mathbb{C}} \mathcal{Q}\left(v,-1^{|v|+4}\right)=\ell(v)+|v|+2$ and $|\nu|$ and $\ell(v)$ are defined in equations (2.1) and (2.5), respectively.

Theorem 2.5 is proved at the end of Section 4.8 with the exception of the explicit value (2.7) for $\operatorname{cyl}_{1}\left(\mathcal{Q}\left(v,-1^{|v|+4}\right)\right)$ which is proved in Section 4.10. For the case of the principal stratum corresponding to $v=\left[1^{k}\right]$, the formula becomes even more explicit; see (4.33).

By a symmetry argument, we get the same asymptotics (2.7) with the same constant $\operatorname{cyl}_{k}\left(\mathcal{Q}\left(v,-1^{|v|+4}\right)\right)$ for the number of square-tiled surfaces with $k$ vertical (instead of horizontal) bands of squares.

We have seen that meanders are in bijective correspondence with squaretiled surfaces whose associated horizontal and vertical multicurves have a single component. The following result transfers to meanders the explicit asymptotics from Theorem 2.5.

THEOREM 2.6. The number $\mathcal{S}_{k_{h}, k_{v}, v}^{\text {labeled }}(N)$ of square-tiled surfaces in the stratum $\mathcal{Q}\left(v,-1^{|v|+4}\right)$ with labeled zeros and poles tiled with at most $2 N$ squares composed of $k_{h}$ horizontal and $k_{v}$ vertical bands of squares (that is, having the associated horizontal multicurve consisting of $k_{h}$ components and the associated vertical multicurve consisting of $k_{v}$ components) has the following asymptotics as $N \rightarrow+\infty$ :

$$
\mathcal{S}_{k_{h}, k_{v}, v}^{\text {labeled }}(N)=\operatorname{cyl}_{k_{h}, k_{v}}\left(\mathcal{Q}\left(\nu,-1^{|v|+4}\right)\right) \cdot \frac{N^{d}}{2 d}+o\left(N^{d}\right) \text { as } N \rightarrow+\infty,
$$

where the constant $\operatorname{cyl}_{k_{h}, k_{v}}\left(\mathcal{Q}\left(\nu,-1^{|v|+4}\right)\right)$ satisfies the following relation:

$$
\frac{\operatorname{cyl}_{k_{h}, k_{v}}\left(\mathcal{Q}\left(v,-1^{|v|+4}\right)\right)}{\operatorname{Vol}_{1} \mathcal{Q}_{1}\left(v,-1^{|v|+4}\right)}=\frac{\operatorname{cyl}_{k_{h}}\left(\mathcal{Q}\left(v,-1^{|v|+4}\right)\right)}{\operatorname{Vol}_{1} \mathcal{Q}_{1}\left(v,-1^{|v|+4}\right)} \cdot \frac{\operatorname{cyl}_{k_{v}}\left(\mathcal{Q}\left(v,-1^{|v|+4}\right)\right)}{\operatorname{Vol}_{1} \mathcal{Q}_{1}\left(v,-1^{|v|+4}\right)},
$$

and the constants $\operatorname{cyl}_{k_{h}}\left(\mathcal{Q}\left(v,-1^{|v|+4}\right)\right), \operatorname{cyl}_{k_{v}}\left(\mathcal{Q}\left(v,-1^{|v|+4}\right)\right)$ are the ones from Theorem 2.5.

Theorem 2.6 is proved at the end of Section 4.8. The relation (2.9) can be viewed as a statement about independence of horizontal and vertical decompositions of square-tiled surfaces.

Forgetting the labeling of zeros and poles (see Remark 2.4), we get the asymptotics of the number of connected pairs of transverse simple closed curves of fixed combinatorial type with at most $2 \mathrm{~N}$ crossings.

Further remarks. It is worth mentioning that all the above quantities have a combinatorial nature but were computed by alternative methods. The MasurVeech volumes in genus zero $\operatorname{Vol}_{1} \mathcal{Q}_{1}\left(v,-1^{|v|+4}\right)$ are closely related to Hurwitz 
numbers counting covers of the sphere of some very special ramification type. However, all attempts to compute these volumes by purely combinatorial methods have (up to now) failed even for covers of the simplest ramification type; see e.g. [AEZ1]. The proof in [AEZ2] of the formula for the MasurVeech volumes implicitly uses the analytic Riemann-Roch theorem in addition to combinatorics.

Theorem 2.6 about square-tiled surfaces with a fixed horizontal and vertical combinatorics is proved in Section 4 using ergodicity of the $\mathrm{SL}_{2}(\mathbb{R})$-action with respect to the Masur-Veech measure and Moore's ergodicity theorem. The proof was inspired by the approach of Mirzakhani to counting simple closed geodesics on hyperbolic surfaces.

Note that the error term in Theorem 2.5 has the form $O\left(N^{d-1}\right)$, while the error term in Theorem 2.6 has a weaker form $o\left(N^{d}\right)$. The underlying reason is that the count in Theorem 2.5 can be expressed in terms of the Ehrhart quasipolynomial associated with the Euclidean volume of certain rational polytope, see the Proof of Theorem 4.23 in Section 4.8, while the ergodic technique used in the Proof of Theorem 2.6 is insufficient to provide an explicit error term and leaves us with $o\left(N^{d}\right)$. It would be very interesting to provide a more precise error term in (2.8). The recent paper [EMiMo2] might be very useful in this context. The authors solve a very similar problem, namely they prove a quantitative estimate, with a power saving error term, for the number of simple closed geodesics of length at most $L$ on a closed hyperbolic surface of genus $g$. The relation between counting of square-tiled surfaces and counting simple closed geodesics is described in [DGZZ3].

Let us mention that in two more situations that share many similarities with ours, explicit error terms have been recently carried out: flat geodesics in translation surfaces in [NRW] and the intersection of stable and unstable horospheres in the space $\mathrm{SL}_{d}(\mathbb{R}) / \mathrm{SL}_{d}(\mathbb{Z})$ in $[\mathbf{E H L}]$.

\section{From arc systems and meanders to square-tiled surfaces}

In this section, we give precise bijections between meanders and square-tiled surfaces with a single maximal cylinder in both horizontal and vertical directions. We consider meanders in the plane in Sections 3.1-3.3 and meanders on the sphere in Section 3.4. All the proofs of asymptotic results are based on the results of Section 4.

3.1. Orientation, marking and weight. We have seen in Proposition 2.2 from Section 2.1 that transverse connected pairs of multicurves on the sphere are in bijection with square-tiled surfaces of genus 0 . A square-tiled surface arising 
from a pair of arc systems has a single horizontal band of squares. In particular, a square-tiled surface arising from a meander has a single horizontal and a single vertical band of squares.

However, pairs of arc systems and meanders (both in the plane and on the sphere) carry an extra marking. Namely, a pair of arc systems comes with a given choice of top and bottom sides. Furthermore, the square-tiled surface corresponding to a plane meander has a special square corresponding to the leftmost intersection. Summarizing, we get the following result.

LEMMA 3.1. There is a natural bijection between meanders in the plane and square-tiled surfaces with a marked oriented vertical side of one of the squares that have a single horizontal and a single vertical band of squares.

In order to provide the exact count of meanders, we present the conventions for the count of square-tiled surfaces and see how these quantities are related to arc systems and meander count. We will consider square-tiled surfaces with a marked vertex of the square tiling.

Convention 3.2. By convention, the marked vertex is located at the end of the marked oriented vertical edge on the top boundary component of the single horizontal cylinder.

Note that the two boundary components of the single horizontal cylinder do not intersect. Thus, the marked vertex uniquely defines the top boundary component and, hence, provides us with the canonical orientation of the waist curve of the single horizontal cylinder.

Let us reconstruct the labeled pair of arc systems in the plane from a squaretiled surface of genus zero tiled with a single horizontal band of squares and having a marked vertex. If the marked vertex of the square tiling is a simple pole of the quadratic differential, there is a single vertical side of the square tiling incident to it, and the choice of the vertical side is canonical. If the marked vertex of the square tiling is a regular point of the quadratic differential, there are two adjacent vertical sides; so there are two ways to choose a distinguished vertical side which, generally, lead to two different arc systems. We say 'generally' because it might happen that the square-tiled surface is particularly symmetric (like square-tiled surfaces associated with arc systems from Figure 3) and the resulting two arc systems are isomorphic.

As soon as we are interested only in the asymptotic count, we can simply neglect this issue: the square-tiled surfaces with extra symmetries occur too rarely to affect the asymptotics. To perform the exact count, we establish the following standard convention. 
CONVENTION 3.3. We always count a marked or nonmarked square-tiled surface with a weight reciprocal to the order $\mid$ Aut $\mid$ of its automorphism group. In the current context, we keep track of which sides of the square-tiled surface are horizontal and which ones are vertical, but we do not label either the sides or the vertices of the square-tiled surface. By definition, the automorphism group Aut acts by flat isometries sending horizontal (respectively vertical) sides of the tiling to horizontal (respectively vertical) sides and keeping the marked point (if any) fixed.

In particular, if we have a marked point at a regular vertex of a square-tiled surface, the automorphism group is either trivial or $\mathbb{Z} / 2 \mathbb{Z}$. If we have a marked point at a zero of degree $j$ of a square-tiled surface, the automorphism group is a (usually trivial) subgroup of the cyclic group $\mathbb{Z} /(j+2) \mathbb{Z}$.

\subsection{Meanders with a given number of minimal arcs and square-tiled} surfaces. In this section and in the next one, we continue to work with plane meanders. Under Conventions 3.2 and 3.3, any collection of weighted squaretiled surfaces on the sphere with a single band of horizontal squares and with a marked regular point defines twice as many arc systems; the weighted collection of square-tiled surfaces as above with a marked zero of degree $j$ defines $(j+2)$ times more arc systems for any $j \in \mathbb{N}$.

LEMMA 3.4. Let the initial meander in the plane have $p$ minimal arcs, where $p \geqslant 3$. The associated square-tiled surface has $p+1$ simple poles if the initial meander has a maximal arc and $p$ simple poles if it does not.

Proof. A maximal arc becomes indistinguishable from a minimal arc after passing to a labeled pair of transverse simple closed curves on the sphere. Minimal and maximal arcs are in bijective correspondence with bigons in the partition of the sphere by the union of these transverse simple closed curves. Bigons, in turn, are in bijective correspondence with simple poles of the associated square-tiled surface.

Recall that $\mathcal{M}_{p}^{+}(N)$ and $\mathcal{M}_{p}^{-}(N)$ denote the number of meanders with $p$ minimal arcs and with or without a maximal arc, respectively. Denote by $\mathcal{P}_{p}(N)$ the number of square-tiled surfaces of genus zero tiled with at most $2 N$ identical squares, having exactly $p$ simple poles and having a single horizontal and a single vertical band of squares. Denote by $\mathcal{P}_{p, j}(N)$, where $j=0,1,2, \ldots$, the number of square-tiled surfaces as above having in addition a marked point at a regular vertex when $j=0$ and at a zero of order $j$ when $j>0$. 
Note that a square-tiled surface of genus 0 with $p$ simple poles cannot have zeros of order greater than $p-4$.

LEMMA 3.5. Under Convention 3.3 on the weighted count of square-tiled surfaces, the following equalities hold:

$$
\begin{aligned}
& \mathcal{M}_{p}^{+}(N)=2(p+1) \cdot \mathcal{P}_{p+1}(N) \\
& \mathcal{M}_{p}^{-}(N)=\sum_{j=0}^{p-4}(j+2) \cdot \mathcal{P}_{p, j}(N)-\frac{1}{2} \mathcal{M}_{p-1}^{+}(N) .
\end{aligned}
$$

Proof. If the meander has $2 n$ intersections, then the associated square-tiled surface is tiled with $2 n$ identical squares.

To every meander with a maximal arc and with $p$ minimal arcs, we associated a canonical square-tiled surface of genus zero with $p+1$ simple poles, a single horizontal and a single vertical band of squares; see Proposition 2.2. Conversely, to every such square-tiled surface, we can associate $2(p+1)$ meanders with one maximal arc and $p$ minimal arcs. Indeed, choose any of the $(p+1)$ simple poles and choose independently one of the two possible orientations of the waist curve of the horizontal cylinder. Cutting this waist curve at the intersection with the single vertical edge of the square tiling adjacent to the selected pole, we get a meander in the plane with a maximal arc.

It might happen that some of the resulting $2(p+1)$ meanders are pairwise isomorphic. However, this implies that the automorphism group of the squaretiled surface is nontrivial, and Convention 3.3 provides the exact count. This completes the proof of equality (3.1).

Similarly, to every meander without a maximal arc and with $p$ minimal arcs, we assigned a canonical square-tiled surface of genus zero having $p$ simple poles, a single horizontal and a single vertical band of squares, and a marked vertex following Convention 3.2. The assumption that the initial meander does not have a maximal arc excludes coincidence of the marked point with a simple pole on the upper side. In order to exclude a maximal arc on the lower side, one needs to subtract a half of $\mathcal{M}_{p-1}^{+}(N)$. At the end of Section 3.1, we have seen that under Convention 3.3 on weights with which we count square-tiled surfaces with a marked vertex, any collection of weighted genus zero squaretiled surfaces with a single horizontal and a single vertical band of squares, and with a marked vertex of the tiling that is regular in the flat metric, defines twice as much meanders in the plane. A weighted collection of square-tiled surfaces as above with a marked zero of degree $j$ defines $(j+2)$ times more meanders in the plane for any $j \in \mathbb{N}$. As before, if some of the resulting meanders are isomorphic, we do not count them several times since by definition of the automorphism 
group Aut of the corresponding square-tiled surface, the resulting multiplicity coincides with the order $\mid$ Aut $\mid$ of the automorphism group. This completes the proof of equality (3.2).

3.3. Meanders and square-tiled surfaces in a given stratum. We now introduce finer count with respect to a fixed stratum of meromorphic quadratic differentials. For a partition $v=\left[1^{v_{1}} 2^{v_{2}} \ldots\right]$, denote by $\mathcal{M}_{v}^{+}(N)$ and $\mathcal{M}_{v}^{-}(N)$ the number of meanders (with and without a maximal arc, respectively) giving rise to square-tiled surfaces in the stratum $\mathcal{Q}^{\text {nonlabeled }}\left(v,-1^{|v|+4}\right)$. In the current setting, we do not label zeros and poles of quadratic differentials. We say that such meanders are of type $v$. Similarly, let $\mathcal{P}_{v}(N)$ be the number of genus zero square-tiled surfaces in the stratum $\mathcal{Q}^{\text {nonlabeled }}\left(\nu,-1^{|v|+4}\right)$ tiled with at most $2 N$ identical squares, with a single horizontal and a single vertical band of squares. Denote by $\mathcal{P}_{v, j}(N), j=0,1,2, \ldots$, the number of square-tiled surfaces as above having, in addition, a marked point at a regular vertex when $j=0$ and at a zero of order $j$ when $j>0$. By definition, we let $\mathcal{P}_{v, j}(N)=0$ for any $N$ when $v_{j}=0$. Recall that by Convention 3.3, we count square-tiled surfaces with weights reciprocal to the orders of their automorphism groups.

LEMMA 3.6. Under Convention 3.3 on weights with which we count squaretiled surfaces, the following equalities hold

$$
\begin{aligned}
& \mathcal{M}_{v}^{+}(N)=2(|v|+4) \cdot \mathcal{P}_{\nu}(N) \\
& \mathcal{M}_{v}^{-}(N)=\sum_{j=0}^{|v|}(j+2) \cdot \mathcal{P}_{\nu, j}(N)-\frac{1}{2} \mathcal{M}_{v}^{+}(N) .
\end{aligned}
$$

Proof. The proof is completely analogous to the Proof of Lemma 3.5.

3.4. Asymptotic density of meanders: general setting. In this section, we return to meanders on the sphere. Let $\mathcal{T}$ be a plane tree. We associate to $\mathcal{T}$ a generalized integer partition $v=v(\mathcal{T})=\left[0^{v_{0}} 1^{v_{1}} 2^{v_{2}} \ldots\right]$, where $v_{j}$ denotes the number of vertices of valence $j+2$ for $j=0,1,2 \ldots$ The number of leaves, or, equivalently, of vertices of valence 1 , is then expressed in terms of the (generalized) partition $v$ as $|v|+4$, where $|v|=1 \cdot v_{1}+2 \cdot v_{2}+3 \cdot v_{3}+\cdots$.

Given two generalized partitions $\iota=\left[0^{\iota_{0}} 1^{\iota_{1}} 2^{\iota_{2}} \ldots\right]$ and $\kappa=\left[0^{\kappa_{0}} 1^{\kappa_{1}} 2^{\kappa_{2}} \ldots\right]$, we define their sum as $v=\iota+\kappa=\left[0^{\iota_{0}+\kappa_{0}} 1^{\iota_{1}+\kappa_{1}} 2^{\iota_{2}+\kappa_{2}} \ldots\right]$. We say that $\iota$ is a subpartition of $v$ if for all $i \geqslant 0$, we have $\iota_{i} \leqslant v_{i}$. We use notation $\iota \subset v$ to indicate that $\iota$ is a subpartition of $v$ and define the difference $\kappa=v-\iota$ in the natural way. 
We formulate and prove the following generalization of Theorem 1.2 giving a formula for the limit of the fraction (1.3) of meanders among all gluings which we get identifying arc systems of types $\mathcal{T}_{\text {top }}$ and $\mathcal{T}_{\text {bottom }}$ with the same number of arcs; see Figure 3.

Though we agreed in Section 1.3 to consider reduced trees, suppressing the vertices of valence 2 , it is often convenient to keep several marked points; so we state the following theorem in this slightly more general setting. Note that since $f(0)=2$, the number $v_{0}$ of zeros in the partition $v$ affects the value of the function $\operatorname{Vol}_{1} \mathcal{Q}_{1}\left(\nu,-1^{|v|+4}\right)$. Adding an extra marked point, we double the Masur-Veech volume of the corresponding stratum.

THEOREM 3.7. For any pair of plane trees $\mathcal{T}_{\text {top }}, \mathcal{T}_{\text {bottom }}$ with associated generalized partitions $v_{\text {top }}$ and $v_{\text {bottom, we have }}$

$$
\lim _{N \rightarrow+\infty} \mathrm{P}_{\text {connected }}\left(\mathcal{T}_{\text {top }}, \mathcal{T}_{\text {bottom }} ; N\right)=\mathrm{P}_{1}\left(\mathcal{Q}\left(\nu,-1^{|v|+4}\right)\right)>0,
$$

where $v=v_{\text {top }}+v_{\text {bottom }}$ and

$$
\mathrm{P}_{1}\left(\mathcal{Q}\left(v,-1^{|v|+4}\right)\right)=\frac{\operatorname{cyl}_{1}\left(\mathcal{Q}\left(v,-1^{|v|+4}\right)\right)}{\operatorname{Vol}_{1} \mathcal{Q}_{1}\left(\nu,-1^{|v|+4}\right)} .
$$

Here

$$
\operatorname{cyl}_{1}\left(\mathcal{Q}\left(\nu,-1^{|v|+4}\right)\right)=2 \sum_{\mu \subset v}\left(\begin{array}{l}
|v|+4 \\
|\mu|+2
\end{array}\right)\left(\begin{array}{c}
v_{0} \\
\mu_{0}
\end{array}\right)\left(\begin{array}{c}
v_{1} \\
\mu_{1}
\end{array}\right)\left(\begin{array}{c}
v_{2} \\
\mu_{2}
\end{array}\right) \ldots
$$

and $\mathrm{Vol}_{1} \mathcal{Q}_{1}\left(\nu,-1^{|v|+4}\right)$ is given by (2.2).

Proof. Under our correspondence which associates with pairs of transverse multicurves square-tiled surfaces, the trees $\mathcal{T}_{\text {top }}$ and $\mathcal{T}_{\text {bottom }}$ represent the trees formed by the horizontal saddle connections of the square-tiled surface (see Section 4.10 for details).

Vertices of valence 1 are in bijective correspondence with simple poles. Vertices of valence 2 represent marked points (if any). Vertices of valence $j+2$ are in bijective correspondence with zeros of degree $j$ for $j \in \mathbb{N}$. Recall that the type $v=\left[1^{v_{1}} 2^{v_{2}} 3^{v_{3}} \ldots\right]$ of the graph $\mathcal{T}_{\text {top }} \sqcup \mathcal{T}_{\text {bottom }}$ encodes the total number $v_{j}$ of vertices of valence $j+2$ in $\mathcal{T}_{\text {top }} \sqcup \mathcal{T}_{\text {bottom }}$ for $j \in \mathbb{N}$. We conclude that a pair of arc systems having $\mathcal{T}_{\text {top }}$ and $\mathcal{T}_{\text {bottom }}$ as dual trees defines a square-tiled surface in the stratum $\mathcal{Q}^{\text {nonlabeled }}\left(v,-1^{|v|+4}\right)$ of meromorphic quadratic differentials.

We are ready to express the numerator and the denominator of the right-hand side in (1.3) in terms of square-tiled surfaces. First, note that arc systems are 
defined on a pair of labeled oriented discs (called top and bottom, or northern and southern hemispheres).

Every triple

( $n$-arc system of type $\mathcal{T}_{\text {top }} ; n$-arc system of type $\mathcal{T}_{\text {bottom }} ;$ identification)

in the denominator in (1.3) defines a unique isomorphism class of ordered transverse connected pairs of multicurves in the sense of Definition 2.1 such that the 'horizontal' multicurve is connected and oriented, and the number of intersections is equal to $2 n$. We identify triples leading to isomorphic pairs of labeled multicurves. Pairs of multicurves associated with the triples from the numerator of (1.3) are distinguished by the property that both 'horizontal' and 'vertical' multicurves are connected.

The horizontal saddle connections of a square-tiled surface form a graph $\Gamma$ embedded in the underlying topological surface, that is, a ribbon graph. The vertices of $\Gamma$ coincide with the zeros and poles of the differential. As in the case of dual graph $\mathcal{T}_{\text {top }} \sqcup \mathcal{T}_{\text {bottom }}$, vertices of $\Gamma$ of valence $j+2$ are in bijective correspondence with zeros of degree $j$. We call $\Gamma$ the diagram of horizontal saddle connections of the square-tiled surface.

Applying Proposition 2.2 and constructions of Section 2.1, we associate square-tiled surfaces to the resulting transverse connected pairs of multicurves. We get a surjective map from the triples in the denominator of (1.3) to the set of square-tiled surfaces in the stratum $\mathcal{Q}^{\text {nonlabeled }}\left(\nu,-1^{|v|+4}\right)$ having a single horizontal band of squares and having the ribbon graph $\Gamma:=\mathcal{T}_{\text {top }} \sqcup \mathcal{T}_{\text {bottom }}$ as the diagram of horizontal saddle connections. We denote the number of such square-tiled surfaces tiled with at most $2 N$ identical squares by $\mathcal{S}_{\Gamma, 1, v}(N)$.

Restricting the above map to the triples in the numerator of (1.3), we get a surjective map to the subset of square-tiled surfaces as above which have a single horizontal and a single vertical band of squares. We denote the number of such square-tiled surfaces tiled with at most $2 N$ identical squares by $\mathcal{S}_{\Gamma, 1,1, v}(N)$.

Note that the waist curve of the horizontal cylinder of the resulting square-tiled surface is not oriented, while the boundary circle of each arc system in the disc is oriented. When $\mathcal{T}_{\text {top }}$ and $\mathcal{T}_{\text {bottom }}$ are not isomorphic as ribbon graphs, there is a canonical way to orient the waist curve of the single horizontal cylinder: choose the orientation of the vertical direction so that $\mathcal{T}_{\text {top }}$ is on top of the cylinder; the canonical orientation of the cylinder determines the orientation of the waist curve. In this case, the constructed map is a bijection.

When $\mathcal{T}_{\text {top }}$ and $\mathcal{T}_{\text {bottom }}$ are isomorphic, a negligible part of square-tiled surfaces as above has extra symmetry, namely, an isometry interchanging the two components of the cylinder. Such isometry changes the orientation of the horizontal waist curve. When $\mathcal{T}_{\text {top }}$ and $\mathcal{T}_{\text {bottom }}$ are isomorphic, we count square- 
tiled surfaces in the image of the above map with weight $1 / 2$ when the squaretiled surface is symmetric in the above sense and with weight 1 otherwise. In this case, both the numerator and the denominator in (1.3) give twice the weighted count of the associated square-tiled surfaces.

Thus, the limit in Theorem 3.7 can be expressed as follows:

$$
\lim _{N \rightarrow+\infty} \mathrm{P}_{\text {connected }}\left(\mathcal{T}_{\text {top }}, \mathcal{T}_{\text {bottom }} ; N\right)=\lim _{N \rightarrow+\infty} \frac{\mathcal{S}_{\Gamma, 1,1, v}(N)}{\mathcal{S}_{\Gamma, 1, v}(N)} .
$$

It will be convenient to pass to the quantities $\mathcal{S}_{\Gamma, 1,1, v}^{\text {labeled }}(N)$ and $\mathcal{S}_{\Gamma, 1, v}^{\text {labeled }}(N)$ counting the square-tiled surfaces as above, but with labeled zeros and poles. By Remark 2.4, they differ from $\mathcal{S}_{\Gamma, 1,1, v}(N)$ and $\mathcal{S}_{\Gamma, 1, v}(N)$ by the common constant factor, and, hence, we get the same ratio as above. By Lemma 4.27 at the end of Section 4.8, we have

$$
\lim _{N \rightarrow+\infty} \frac{\mathcal{S}_{\Gamma, 1,1, v}^{\text {labeled }}(N)}{\mathcal{S}_{\Gamma, 1, v}^{\text {labeled }}(N)}=\lim _{N \rightarrow+\infty} \frac{\mathcal{S}_{1, v}^{\text {labeled }}(N)}{\mathcal{S}_{v}^{\text {labeled }}(N)} .
$$

The above formula is just another manifestation of the general principal according to which 'horizontal and vertical cylinder decompositions are asymptotically uncorrelated': defining the counting functions on the righthand side of the above equation, we omit the conditions on the horizontal decomposition imposed in the definition of the corresponding counting functions on the left-hand side.

Applying (2.3) and (2.6) for the numerator and the denominator of the latter fraction, respectively, we prove that the limit in the left-hand side of (3.7) exists and that its value is given by (3.5). The remaining formula (3.6) is equivalent to formula (2.7) from Theorem 2.5 which will be proved in Section 4.10.

Proof of Theorem 1.2. Theorem 1.2 is a particular case of the Theorem 3.7 when the plane trees $\mathcal{T}_{\text {top }}, \mathcal{T}_{\text {bottom }}$ are trivalent and have the total number $p$ of leaves (vertices of valence 1). In this situation, $v=\left[1^{p-4}\right]$. By Theorem 3.7, we have

$$
\lim _{N \rightarrow+\infty} \mathrm{P}_{\text {connected }}\left(\mathcal{T}_{\text {top }}, \mathcal{T}_{\text {bottom }} ; N\right)=\mathrm{P}_{1}\left(\mathcal{Q}\left(1^{p-4},-1^{p}\right)\right)=\frac{\operatorname{cyl}_{1}\left(\mathcal{Q}\left(1^{p-4},-1^{p}\right)\right)}{\operatorname{Vol}_{1} \mathcal{Q}_{1}\left(1^{p-4},-1^{p}\right)} .
$$

It remains to apply (2.2) and (4.33), respectively, for the denominator and the numerator of the latter fraction to complete the proof. Formula (4.33) will be proved in Section 4.10.

3.5. Counting meanders of special combinatorial types. In this section, we return to plane meanders with exception for the Proof of Theorem 1.3 at the very end of the section, where we work with meanders on the sphere. 
We state now an analog of Theorem 1.1, where instead of the number of minimal arcs (pimples), we use the partition $v$ as a combinatorial passport of the meander.

THEOREM 3.8. For any partition $v=\left[1^{v_{1}} 2^{v_{2}} 3^{v_{3}} \ldots\right]$, the number $\mathcal{M}_{v}^{+}(N)$ (respectively $\left.\mathcal{M}_{v}^{-}(N)\right)$ of plane meanders of type $v$, with (respectively without) a maximal arc and with at most $2 \mathrm{~N}$ crossings has the following asymptotics as $N \rightarrow+\infty$ :

$$
\begin{aligned}
\mathcal{M}_{v}^{+}(N)= & 2(|v|+4) \cdot \frac{\operatorname{cyl}_{1,1}\left(\mathcal{Q}\left(v,-1^{|v|+4}\right)\right)}{(|v|+4) ! \cdot \prod_{j} v_{j} !} \cdot \frac{N^{\ell(v)+|v|+2}}{2 \ell(v)+2|v|+4} \\
& +o\left(N^{\ell(v)+|v|+2}\right), \\
\mathcal{M}_{v}^{-}(N)= & \frac{2 \operatorname{cyl}_{1,1}\left(\mathcal{Q}\left(v, 0,-1^{|v|+4}\right)\right)}{(|v|+4) ! \cdot \prod_{j} v_{j} !} \cdot \frac{N^{\ell(v)+|v|+3}}{2 \ell(v)+2|v|+6} \\
& +o\left(N^{\ell(v)+|v|+3}\right) .
\end{aligned}
$$

Moreover, we have

$$
\begin{aligned}
& \operatorname{cyl}_{1,1}\left(\mathcal{Q}\left(v,-1^{|v|+4}\right)\right) \\
& \quad=\frac{4}{\operatorname{Vol}_{1} \mathcal{Q}_{1}\left(\nu,-1^{|v|+4}\right)} \cdot\left(\sum_{\iota_{1}=0}^{v_{1}} \sum_{\iota_{2}=0}^{v_{2}} \sum_{\ldots}^{\ldots}\left(\begin{array}{l}
v_{1} \\
\iota_{1}
\end{array}\right)\left(\begin{array}{c}
v_{2} \\
\iota_{2}
\end{array}\right) \cdots\left(\begin{array}{c}
|v|+4 \\
|\iota|+2
\end{array}\right)\right)^{2}
\end{aligned}
$$

and

$$
\operatorname{cyl}_{1,1}\left(\mathcal{Q}\left(\nu, 0,-1^{|v|+4}\right)\right)=2 \cdot \operatorname{cyl}_{1,1}\left(\mathcal{Q}\left(\nu,-1^{|v|+4}\right)\right) .
$$

Note that contrary to the original Theorem 1.1, where the setting is somewhat misleading, in the setting of Theorem 3.8, we get a more natural formula $\mathcal{M}_{v}^{+}(N)=o\left(\mathcal{M}_{v}^{-}(N)\right)$ as $N \rightarrow+\infty$.

Up to now, we performed the exact count. The lemma below gives the term with dominating contribution to the asymptotic count when the bound $2 \mathrm{~N}$ for the number of squares in the square-tiled surface tends to infinity.

LEMMA 3.9. We have the following limits:

$$
\begin{aligned}
& \lim _{N \rightarrow+\infty} \frac{1}{\mathcal{P}_{1^{p-4}}(N)} \cdot \mathcal{P}_{p}(N)=1, \\
& \lim _{N \rightarrow+\infty} \frac{1}{2 \mathcal{P}_{1^{p-4}, 0}(N)} \cdot\left(\sum_{j=0}^{p-4}(j+2) \cdot \mathcal{P}_{p, j}(N)\right)=1,
\end{aligned}
$$




$$
\lim _{N \rightarrow+\infty} \frac{1}{2 \mathcal{P}_{\nu, 0}(N)} \cdot\left(\sum_{j=0}^{|v|}(j+2) \cdot \mathcal{P}_{\nu, j}(N)\right)=1
$$

Proof. Let $v=\left[1^{v_{1}} 2^{v_{2}} \ldots\right]$ be a partition of $p-4$. By definition, $\mathcal{P}_{v}(N)$ and $\mathcal{S}_{1,1, v}(N)$ denote the same quantities. Using Remark 2.4 to express $\mathcal{S}_{1,1, v}(N)$ in terms of $\mathcal{S}_{1,1, v}^{\text {labeled }}(N)$ and applying (2.8) with $k_{h}=k_{v}=1$, we get

$$
\begin{aligned}
\mathcal{P}_{v}(N) & =\mathcal{S}_{1,1, v}(N)=\frac{1}{p ! \cdot \prod_{j} v_{j} !} \cdot \mathcal{S}_{1,1, v}^{\text {labeled }}(N) \\
& =\frac{\operatorname{cyl}_{1,1}\left(\mathcal{Q}\left(v,-1^{p}\right)\right)}{p ! \cdot \prod_{j} v_{j} !} \cdot \frac{N^{d}}{2 d}+o\left(N^{d}\right), \text { when } N \rightarrow+\infty,
\end{aligned}
$$

where $d$ is defined in (2.4).

For a given number $p \geqslant 4$ of simple poles, the only stratum of the maximal dimension $2 p-6$ is the principal stratum $\mathcal{Q}\left(1^{p-4},-1^{p}\right)$, for which all zeros of the quadratic differentials are simple. Thus, this is the only stratum which contributes a term of order $N^{2 p-6}$ to $\mathcal{P}_{p}(N)$. This proves (3.12).

For $j \geqslant 1$, the quantity $\mathcal{P}_{v, j}(N)$ counts square-tiled surfaces with a marked zero of order $j$ in the stratum $\mathcal{Q}^{\text {nonlabeled }}\left(v,-1^{|v|+4}\right)$. Hence, it has the asymptotic growth rate of the same order as the quantity $\mathcal{P}_{v}(N)$ counting unmarked squaretiled surfaces in the same stratum, that is, it grows like $N^{d}$, where $d=\operatorname{dim}_{\mathbb{C}} \mathcal{Q}(v$, $\left.-1^{|v|+4}\right)$. The dimensional count as above implies that the contribution of any term $\mathcal{P}_{v, j}(N)$ with $j \geqslant 1$ to the sum in the right-hand side of (3.13) has the order at most $N^{2 p-6}$.

Let us analyze now the contribution of various strata to $\mathcal{P}_{p, 0}(N)$. In the same way as we got (3.15), we obtain

$$
\begin{aligned}
\mathcal{P}_{v, 0}(N)= & \frac{\operatorname{cyl}_{1,1}\left(\mathcal{Q}\left(\nu, 0,-1^{|v|+4}\right)\right)}{(|\nu|+4) ! \cdot \prod_{j} v_{j} !} \cdot \frac{N^{\ell(v)+|v|+3}}{2 \ell(\nu)+2|v|+6} \\
& +o\left(N^{\ell(v)+|v|+3}\right), \text { when } N \rightarrow+\infty,
\end{aligned}
$$

where the constant $\operatorname{cyl}_{1,1}\left(\mathcal{Q}\left(v, 0,-1^{|v|+4}\right)\right)$ is strictly positive. This implies that for any partition $v$ of $p-4$ different from $1^{p-4}$, its contribution $\mathcal{P}_{v, 0}$ also has order at most $N^{2 p-6}$. We conclude that $\mathcal{P}_{p, 0}(N)$ has order $N^{2 p-5}$ for $N$ large and that the only stratum which gives a contribution of this order is the principal stratum with a marked point $\mathcal{Q}\left(1^{p-4}, 0,-1^{p}\right)$. This proves equality (3.13).

By the same reason, the summand $2 \mathcal{P}_{v, 0}(N)$ dominates in the sum in the righthand side of (3.14). It is the only term whose contribution is of order $N^{d+1}$, where $d=\operatorname{dim}_{\mathbb{C}} \mathcal{Q}\left(v,-1^{|v|+4}\right)$. The asymptotics of the other terms in the sum have lower orders in $N$ as $N \rightarrow+\infty$. This proves equality (3.14). 
Now we have everything for the proofs of Theorems 1.1 and 3.8.

Proof of Theorem 1.1. The chain of relations starting with (3.1) and continuing with (3.12) and (3.15) yields

$$
\begin{aligned}
\mathcal{M}_{p}^{+}(N) & =2(p+1) \cdot \mathcal{P}_{p+1}(N)=2(p+1) \cdot \mathcal{P}_{1^{p-3}}(N)+o\left(N^{2 p-4}\right) \\
& =2(p+1) \cdot \frac{\operatorname{cyl}_{1,1}\left(\mathcal{Q}\left(1^{p-3},-1^{p+1}\right)\right)}{(p+1) !(p-3) !} \cdot \frac{N^{2 p-4}}{4 p-8}+o\left(N^{2 p-4}\right)
\end{aligned}
$$

when $N \rightarrow+\infty$.

This proves the first equality in (1.1). The constant $\operatorname{cyl}_{1,1}\left(\mathcal{Q}\left(1^{p-3},-1^{p+1}\right)\right)$ is expressed by Formula (2.9) in terms of $\operatorname{cyl}_{1}\left(\mathcal{Q}\left(1^{p-3},-1^{p+1}\right)\right)$ computed in Corollary 4.30 of Section 4.10 and in terms of the Masur-Veech volume of the stratum $\mathcal{Q}\left(1^{p-3},-1^{p+1}\right)$ given by Formula (2.2).

Similarly, the chain of relations including (3.2), (3.13) and (3.16) implies

$$
\begin{gathered}
\mathcal{M}_{p}^{-}(N)=\sum_{j=0}^{p-4}(j+2) \cdot \mathcal{P}_{p, j}(N)-\frac{1}{2} \mathcal{M}_{p-1}^{+}(N)=2 \mathcal{P}_{1^{p-4}, 0}(N)+o\left(N^{2 p-5}\right) \\
=\frac{2 \operatorname{cyl}_{1,1}\left(\mathcal{Q}\left(1^{p-4}, 0,-1^{p}\right)\right)}{p !(p-4) !} \cdot \frac{N^{2 p-5}}{4 p-10}+o\left(N^{2 p-5}\right), \text { when } N \rightarrow+\infty
\end{gathered}
$$

This proves the first equality in (1.2). The constant $\operatorname{cyl}_{1,1}\left(\mathcal{Q}\left(1^{p-4}, 0,-1^{p}\right)\right)$ is expressed by our main formula (2.9) in terms of $\operatorname{cyl}_{1}\left(\mathcal{Q}\left(1^{p-4}, 0,-1^{p}\right)\right)$ computed in Corollary 4.30 of Section 4.10 and in terms of the Masur-Veech volume of the stratum $\mathcal{Q}\left(1^{p-4}, 0,-1^{p}\right)$ given by formula (2.2).

Thus, the Proof of Theorem 1.1 is conditional subject to the explicit count of $\operatorname{cyl}_{1}\left(\mathcal{Q}\left(1^{p-3},-1^{p+1}\right)\right)$ and of $\operatorname{cyl}_{1}\left(\mathcal{Q}\left(1^{p-4}, 0,-1^{p}\right)\right)$ performed in Corollary 4.30 .

Proof of Theorem 3.8. The Proof of Theorem 3.8 is completely analogous to the Proof of Theorem 1.1.

Combining (3.3) with relation (3.15), we get

$$
\begin{aligned}
\mathcal{M}_{v}^{+}(N)= & 2(|v|+4) \cdot \mathcal{P}_{v}(N) \\
= & 2(|v|+4) \cdot \frac{\operatorname{cyl}_{1,1}\left(\mathcal{Q}\left(v,-1^{|v|+4}\right)\right)}{(|v|+4) ! \cdot \prod_{j} v_{j} !} \cdot \frac{N^{\ell(v)+|v|+2}}{2 \ell(v)+2|v|+4} \\
& +o\left(N^{\ell(v)+|v|+2}\right), \text { when } N \rightarrow+\infty,
\end{aligned}
$$

where we use Formula (2.4) for the dimension $d$ of the stratum $\mathcal{Q}\left(v,-1^{|v|+4}\right)$. This proves formula (3.8) in Theorem 3.8. 
Similarly, combining (3.4) with (3.14) and (3.16), we get

$$
\begin{aligned}
\mathcal{M}_{v}^{-}(N)= & \sum_{j=0}^{|v|}(j+2) \cdot \mathcal{P}_{v, j}(N)-\frac{1}{2} \mathcal{M}_{v}^{+}(N)=2 \mathcal{P}_{v, 0}(N)+o\left(N^{2 \ell(v)+2|v|+3}\right) \\
= & \frac{2 \operatorname{cyl}_{1,1}\left(\mathcal{Q}\left(v, 0,-1^{|v|+4}\right)\right)}{(|v|+4) ! \cdot \prod_{j} v_{j} !} \cdot \frac{N^{\ell(v)+|v|+3}}{2 \ell(v)+2|v|+6} \\
& +o\left(N^{\ell(v)+|v|+3}\right), \text { when } N \rightarrow+\infty
\end{aligned}
$$

This proves formula (3.9) in Theorem 3.8.

We have proved Theorem 3.8 conditional to expressions (3.11) and (3.10) for the quantities $\operatorname{cyl}_{1,1}\left(\mathcal{Q}\left(v, 0,-1^{|v|+4}\right)\right)$ and $\operatorname{cyl}_{1,1}\left(\mathcal{Q}\left(v,-1^{|v|+4}\right)\right)$. We prove them together with relation (2.7) in Section 4.10.

We conclude this section with the Proof of Theorem 1.3.

Proof of Theorem 1.3. Following the same arguments as in the Proof of Theorem 3.7, we obtain

$$
\lim _{N \rightarrow+\infty} \mathrm{P}_{\text {connected }}(p ; N)=\lim _{N \rightarrow+\infty} \frac{\sum_{|v|=p-4} \mathcal{S}_{1,1, v}^{\text {labeled }}(N)}{\sum_{|v|=p-4} \mathcal{S}_{1, v}^{\text {labeled }}(N)},
$$

where $\mathcal{S}_{1, v}^{\text {labeled }}(N)$ and $\mathcal{S}_{1,1, v}^{\text {labeled }}(N)$ are as in Theorems 2.5 and 2.6, respectively. By (2.6) and (2.8), for any partition $v$, the latter quantities have order $N^{d}$, where $d=\operatorname{dim}_{\mathbb{C}} \mathcal{Q}\left(v,-1^{p}\right)$. Among all partitions $v$ satisfying $|v|=p-4$, there is only one partition, namely $\left[1^{p-4}\right]$, which defines the stratum $\mathcal{Q}\left(1^{p-4},-1^{p}\right)$ of maximal dimension $2 p-6$. Hence, the contributions of other strata to both sums are negligible with respect to the contributions of the principal stratum. This implies that

$$
\lim _{N \rightarrow+\infty} \mathrm{P}_{\text {connected }}(p ; N)=\lim _{N \rightarrow+\infty} \frac{\mathcal{S}_{1,1,\left[1^{p-4}\right]}^{\text {labeled }}(N)}{\mathcal{S}_{1,\left[1^{-4}\right]}^{\text {labeled }}(N)} .
$$

Using expressions (2.6) and (2.8) for the denominator and the numerator of the latter fraction, respectively, and applying (2.9), we get

$$
\lim _{N \rightarrow+\infty} \mathrm{P}_{\text {connected }}(p ; N)=\frac{\operatorname{cyl}_{1}\left(\mathcal{Q}\left(1^{p-4},-1^{p}\right)\right)}{\operatorname{Vol}_{1} \mathcal{Q}_{1}\left(1^{p-4},-1^{p}\right)} .
$$

The latter ratio was denoted by $\mathrm{P}_{1}\left(\mathcal{Q}\left(1^{p-4},-1^{p}\right)\right)$ and computed in Theorem 1.2. 


\section{Enumeration of square-tiled surfaces}

In the current section, we prove Theorem 2.6 from Section 1 and, in particular, the key equality (2.9). This result is actually a particular case of the more general Corollary 4.25 that concerns surfaces of any genera.

In plain terms, we prove that the asymptotic proportion of square-tiled surface having a single vertical band of squares among square-tiled surface having a single horizontal band of squares is the same as the asymptotic proportion of square-tiled surface having a single vertical band of squares among all square-tiled squares (when the bound for the number of squares in the square tiling grows). This equality can be seen as a particular case of asymptotic noncorrelation of horizontal and vertical decompositions of square-tiled surfaces tiled with large number of squares. Together with the explicit formula (2.2) for the Masur-Veech volume $\operatorname{Vol}_{1} \mathcal{Q}_{1}\left(v,-1^{|v|+4}\right)$ of any stratum in the moduli space of quadratic differentials in genus zero, the equality (2.9) is the principal ingredient of the Proof of Theorem 3.7, providing the asymptotic frequency of meanders out of general couplings of arc systems on a pair of hemispheres.

Our noncorrelation results are more general in two aspects. On the one hand, they are applicable to much more general horizontal and vertical cylinder decompositions of square-tiled surfaces compared to 'single band of squares' needed for the count of meanders; see Corollaries 4.24 and 4.25 as well as Remark 4.26. On the other hand, these noncorrelation results are applicable to general closed connected $\mathrm{GL}_{2}(\mathbb{R})$-invariant suborbifolds defined over $\mathbb{Q}$ in any genus (and not only to strata of quadratic differentials in genus zero needed for meanders).

4.1. Invariant arithmetic orbifolds. Given a generalized partition $\kappa$ composed of nonnegative integers $\kappa=\left[0^{\kappa_{0}} 1^{\kappa_{1}} \ldots\right]$ satisfying $\sum_{i \geqslant 0} i \kappa_{i}=2 g-2$, we denote by $\mathcal{H}(\kappa)$ the moduli space of Abelian differentials with $\kappa_{i}$ zeros of order $i$, for $i=0,1, \ldots$, on complex curves of genus $g$. Namely, an element of $\mathcal{H}(\kappa)$ is a tuple $\left(X, \omega,\left\{P_{i, j}\right\}_{i \geqslant 0,1 \leqslant j \leqslant \kappa_{i}}\right)$ where $X$ is a smooth complex curve of genus $g$ endowed with a nonzero holomorphic 1-form $\omega$ having zero of order $i$ at each of the points $P_{i, 1}, \ldots, P_{i, \kappa_{i}}$ of $X$ (for those $i$ for which $\kappa_{i}>0$ ) and nonvanishing outside of the finite set $\Sigma:=\left\{P_{i, j}\right\}$. Here, 'zero of order zero' is interpreted as a marked point. Each stratum of Abelian differential is locally modeled on the relative cohomology $H^{1}(X, \Sigma ; \mathbb{C})$.

A translation chart for an Abelian differential $\omega$ is a local coordinate $z$ on $X$ in which $\omega=d z$. A translation chart defines a flat metric with the area form $\frac{i}{2} d z \wedge d \bar{z}=d x \wedge d y$. The group $\mathrm{GL}_{2}(\mathbb{R})$ acts on $\mathcal{H}(\kappa)$ by postcomposition in 
translation charts. By definition,

$$
\forall A \in \mathrm{GL}_{2}(\mathbb{R}), \quad \operatorname{Area}(A \cdot(X, \omega, \Sigma))=\operatorname{det}(A) \cdot \operatorname{Area}((X, \omega, \Sigma)) .
$$

In particular, the subgroup $\mathrm{SL}_{2}(\mathbb{R})$ preserves the 'unit hyperboloid' $\mathcal{H}_{1}(\kappa) \subset$ $\mathcal{H}(\kappa)$ that consists of Abelian differentials of area one.

Definition 4.1. A square-tiled surface in a stratum of Abelian differential $\mathcal{H}(\kappa)$ is an Abelian differential $(X, \omega, \Sigma)$ such that all periods of $\omega$ relative to $\Sigma$ belong to $\mathbb{Z} \oplus i \mathbb{Z}$.

Square-tiled surfaces correspond to integral points $H^{1}(X, \Sigma ; \mathbb{Z} \oplus i \mathbb{Z})$ in period coordinates $H^{1}(X, \Sigma ; \mathbb{C})$. The action of the subgroup $\mathrm{SL}_{2}(\mathbb{Z}) \subset \mathrm{SL}_{2}(\mathbb{R})$ preserves the set of square-tiled surfaces.

Alternatively, a square-tiled surface can be defined as a connected finite ramified cover over the square torus $\mathbb{C} /(\mathbb{Z} \oplus i \mathbb{Z})$ with all ramification points (if any) located over the same branch point of the torus. Thus, in plain terms, a square-tiled translation surface is obtained by gluing a finite set of unit squares endowed with distinguished horizontal and vertical directions by translation respecting these distinguished directions and identifying sides to sides and vertices to vertices.

The fundamental result of Eskin, Mirzakhani and Mohammadi generalizes to closed $\mathrm{SL}_{2}(\mathbb{R})$-invariant subsets all the structures we described for strata.

TheOrem [EMi, EMiMo1]. Let $\mathrm{P} \subset \mathrm{SL}_{2}(\mathbb{R})$ be the subgroup of uppertriangular matrices. For any $S=(X, \omega)$ in any stratum $\mathcal{H}(\kappa)$ of Abelian differentials, the closures $\overline{P \cdot S}$ and $\overline{\mathrm{SL}_{2}(\mathbb{R}) \cdot S}$ of orbits of $S$ under the actions of $\mathrm{P}$ and of $\mathrm{SL}_{2}(\mathbb{R})$ coincide.

The orbit closure $\mathcal{L}=\overline{\mathrm{GL}_{2}(\mathbb{R}) \cdot S}$ is locally described in period coordinates as a finite union of complexifications of linear subspaces in $H^{1}(X, \Sigma ; \mathbb{R})$. Any such $\mathcal{L}$ admits a volume element which is linear in the corresponding linear subspaces of $H^{1}(X, \Sigma ; \mathbb{R})$.

Any ergodic $\mathrm{P}$-invariant probability measure on the 'unit hyperboloid' $\mathcal{H}_{1}(\kappa)$ is always $\mathrm{SL}_{2}(\mathbb{R})$-invariant. It is supported on the intersection $\mathcal{H}_{1}(\kappa)$ with

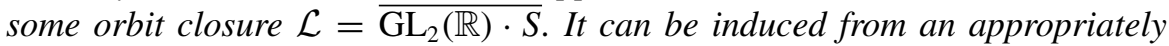
normalized linear volume element in period coordinates of $\mathcal{L}$ by the natural restriction to the level hypersurface $\mathcal{L}_{1}=\mathcal{L} \cap \mathcal{H}_{1}(\kappa)$ of the area function.

Reciprocally, for any orbit closure $\mathcal{L}=\overline{\mathrm{GL}_{2}(\mathbb{R}) \cdot S}$, the measure corresponding to the volume element on $\mathcal{L}_{1}=\mathcal{L} \cap \mathcal{H}_{1}(\kappa)$ induced from any linear volume element in period coordinates of $\mathcal{L}$ by the natural restriction to the level hypersurface $\mathcal{L}_{1}$ is ergodic and finite. 
In the current paper, we will only consider the case when all these linear subspaces are defined by systems of linear equations with rational coefficients with respect to the natural integral structure on $H^{1}(X, \Sigma ; \mathbb{Z}) \subset H^{1}(X, \Sigma ; \mathbb{R})$. In such situation, we say that $\mathcal{L}$ is defined over $\mathbb{Q}$ or, equivalently, that it is arithmetic (see [Wri1] for the more general notion of the field of definition of a $\mathrm{GL}_{2}(\mathbb{R})$-invariant suborbifold).

The following weakened version of results of Wright [Wri2, Theorem 1.9] provides an efficient way to recognize affine invariants suborbifold $\mathcal{L}$ by comparing circumferences of horizontal cylinders of any translation surface in $\mathcal{L}$ having at least one horizontal cylinder.

THEOREM [Wri2]. Suppose that $S$ is a translation surface in a closed connected $\mathrm{GL}_{2}(\mathbb{R})$-invariant submanifold $\mathcal{L}$ and suppose that $S$ has at least one horizontal cylinder. ( $S$ need not be horizontally periodic.) Let $w_{1}, \ldots, w_{n}$ be the set of circumferences of horizontal cylinders on $S$.

If $n=1$, then $\mathcal{L}$ is defined over $\mathbb{Q}$. If $n>1$ but $w_{j} / w_{1} \in \mathbb{Q}$ for all $j=2, \ldots, n$, then $\mathcal{L}$ is defined over $\mathbb{Q}$.

In particular, any connected $\mathrm{GL}_{2}(\mathbb{R})$-invariant suborbifold containing at least one square-tiled surface is defined over $\mathbb{Q}$.

DEFINITION 4.2. By an invariant arithmetic orbifold, we call a closed connected $\mathrm{GL}_{2}(\mathbb{R})$-invariant suborbifold defined over $\mathbb{Q}$ in a stratum of Abelian differentials.

Note that a general invariant arithmetic orbifold might have self-intersections; see [MiWri, Example 2.8] and [LNW, Section 2.1].

Any connected component of any stratum of Abelian differentials is an invariant arithmetic orbifold. The $\mathrm{GL}_{2}(\mathbb{R})$-orbit of any square-tiled surface is also an invariant arithmetic orbifold. Another example, which is of great importance for us, is provided by connected components of strata of meromorphic quadratic differentials with at most simple poles; see Section 4.3.

4.2. Masur-Veech volume element. By definition of the invariant arithmetic orbifold $\mathcal{L}$, the intersection of any linear subspace $L$ representing $\mathcal{L}$ in period coordinates $H^{1}(S, \Sigma ; \mathbb{C})$ with the lattice $H^{1}(S, \Sigma ; \mathbb{Z} \oplus i \mathbb{Z})$ defines a lattice in $L$. Taking the linear volume element in $L$ normalized in such a way that a fundamental domain of the resulting lattice in $L$ is equal to one, we get canonically defined Masur-Veech volume element $d$ Vol on $\mathcal{L}$.

A volume element on a manifold canonically defines a volume element on any level hypersurface of any smooth function. Thus, the Masur-Veech volume element on an invariant arithmetic orbifold $\mathcal{L}$ of a stratum $\mathcal{H}(\kappa)$ of 
Abelian differentials induces the canonical volume element $d \mathrm{Vol}_{1}$ on the "unit hyperboloid' $\mathcal{L}_{1}:=\mathcal{L} \cap \mathcal{H}_{1}(\kappa)$ of Abelian differentials of area one in $\mathcal{L}$.

Alternatively, the volume element $d \mathrm{Vol}_{1}$ (also called the Masur-Veech volume element) on $\mathcal{L}_{1}$ can be defined as follows. Given any subset $U \subset \mathcal{L}$, we define the cones over $U$ as

$$
\begin{aligned}
C_{R} U:=\{(X, r \cdot \omega):(X, \omega) \in U, 0<r \leqslant R\}, \\
C_{\infty} U:=\{(X, r \cdot \omega):(X, \omega) \in U, 0<r\} .
\end{aligned}
$$

Note that $\operatorname{Area}(X, r \cdot \omega)=r^{2} \operatorname{Area}(X, \omega)$ for $r>0$ : the flat area of $(X, r \cdot \omega)$ changes quadratically in $r$. In particular, our definition implies that

$$
C_{R} \mathcal{L}_{1}=\left\{(X, \omega) \in \mathcal{L}: \operatorname{Area}(X, \omega) \leqslant R^{2}\right\} .
$$

By definition, the Masur-Veech volume $\operatorname{Vol}_{1}\left(V_{1}\right)$ of a subset $V_{1} \subset \mathcal{L}_{1}$ of the unit hyperboloid $\mathcal{L}_{1} \subset \mathcal{L}$ is defined as the Masur-Veech volume of the "unit cone' over $V_{1}$ normalized by the dimensional factor $2 d$ :

$$
\operatorname{Vol}_{1} V_{1}=\operatorname{Vol}_{1}\left(V_{1}\right):=2 d \cdot \operatorname{Vol}\left(C_{1} V_{1}\right), \quad \text { where } d=\operatorname{dim}_{\mathbb{C}} \mathcal{L} .
$$

It is immediate to see that the two definitions of the Masur-Veech volume element $d \mathrm{Vol}_{1}$ on an invariant arithmetic suborbifold $\mathcal{L}_{1}$ are equivalent.

By construction, the Masur-Veech volume element thus defined on the unit hyperboloid $\mathcal{L}_{1}$ of any invariant arithmetic suborbifold $\mathcal{L}$ is $\mathrm{SL}_{2}(\mathbb{R})$-invariant. By the fundamental result of Eskin, Mirzakhani, Mohammadi [EMi], [EMiMo1] (see the statement at page 28), for any invariant arithmetic suborbifold $\mathcal{L}$, the measure on $\mathcal{L}_{1}$ represented by Masur-Veech volume element on $\mathcal{L}_{1}$ is a finite ergodic measure. In particular, the total Masur-Veech volume $\mathrm{Vol}_{1} \mathcal{L}_{1}$ of any invariant arithmetic suborbifold $\mathcal{L}$ is finite. (Finiteness of Masur-Veech volumes of the unit hyperboloids of strata of Abelian and quadratic differentials was proved much earlier by Masur [Mas] and Veech [Vee1].)

Given an invariant arithmetic suborbifold $\mathcal{L}$ in the ambient stratum $\mathcal{H}(\kappa)$ of Abelian differentials, denote by $\mathcal{L}_{\mathbb{Z}}$ the set of all square-tiled surfaces in $\mathcal{L}$ tiled with unit squares. Equivalently, $\mathcal{L}_{\mathbb{Z}}$ is the set of points of $\mathcal{L}$ represented in period coordinates of the ambient stratum $\mathcal{H}(\kappa)$ by points of the lattice $H^{1}(S, \Sigma, \mathbb{Z} \oplus$ $i \mathbb{Z})$. It follows from the Definition (4.4) of $\mathrm{Vol}_{1} \mathcal{L}_{1}$ that

$$
\begin{aligned}
\operatorname{Vol}_{1} \mathcal{L}_{1}: & =2 d \cdot \operatorname{Vol}\left(C_{1} \mathcal{L}_{1}\right)=2 d \cdot \lim _{R \rightarrow+\infty} \frac{\operatorname{Card}\left(C_{R} \mathcal{L}_{1} \cap \mathcal{L}_{\mathbb{Z}}\right)}{R^{2 d}} \\
& =2 d \cdot \lim _{N \rightarrow+\infty} \frac{\left(\begin{array}{c}
\text { number of square-tiled surfaces in } \mathcal{L} \\
\text { tiled with at most } N \text { identical squares }
\end{array}\right)}{N^{d}},
\end{aligned}
$$

where $d=\operatorname{dim}_{\mathbb{C}} \mathcal{L}$. 
4.3. Strata of quadratic differentials as invariant arithmetic orbifolds. In this section, we describe strata of meromorphic quadratic differentials and their Masur-Veech volumes. We then explain how this description fits the general setting of invariant arithmetic orbifolds and their Masur-Veech volumes discussed in Sections 4.1-4.2. The details of constructions are deferred to Appendix A.

Similarly to the case of strata $\mathcal{H}(\kappa)$ in the moduli spaces of Abelian differentials introduced in Section 4.1, one can define strata $\mathcal{Q}(\xi)$ in the moduli spaces of meromorphic quadratic differentials with at most simple poles. The strata are parameterized by generalized partitions $\xi=\left[-1^{\xi_{-1}} 0^{\xi_{0}} 1^{\xi_{1}} \ldots\right]$ satisfying the condition $\sum_{i \geqslant-1} i \cdot \xi_{i}=4 g-4$. An element in $\mathcal{Q}(\xi)$ is an equivalence class of tuples $\left(X, q,\left\{P_{i, j}\right\}_{i \geqslant-1,1 \leqslant j \leqslant \xi_{i}}\right)$, where $X$ is a smooth complex curve of genus $g, q$ is a meromorphic quadratic differential on $X$ having simple poles at the points $P_{-1, j}$ of $X$, marked points at the points $P_{0, j}$ of $X$, and zeros of order $i$ at the points $P_{i, j}$ of $X$ for $i \geqslant 1$ and $q$ is not the square of an Abelian differential on $X$. The strata $\mathcal{Q}\left(v,-1^{|v|+4}\right)$ considered in the Section 1 correspond to the case of genus 0 .

By convention, $\left(X, q,\left\{P_{i, j}\right\}\right)$ (which will be sometimes denoted by $(X, q)$ for brevity) defines an integer point in $\mathcal{Q}(\xi)$ if and only if it can be represented by a square-tiled surface tiled by identical squares of size $\frac{1}{2} \times \frac{1}{2}$ such that all points $P_{i, j}$ are located at the corners of the squares. This convention defines a volume form on $\mathcal{Q}(\xi)$ and an induced volume form on $\mathcal{Q}_{1}(\xi)$. The resulting volume $\mathrm{Vol}_{1} \mathcal{Q}_{1}(\xi)$ can be expressed as follows:

$$
\begin{aligned}
& \operatorname{Vol}_{1} \mathcal{Q}_{1}(\xi)=2 d \cdot \lim _{N \rightarrow+\infty} \frac{\left(\begin{array}{c}
\text { number of square-tiled surfaces in } \mathcal{Q}(\xi) \\
\text { tiled with at most } 2 N \text { identical squares }
\end{array}\right)}{N^{d}} \\
& =2 d \cdot 2^{d} \cdot \lim _{N \rightarrow+\infty} \frac{\left(\begin{array}{c}
\text { number of square-tiled surfaces in } \mathcal{Q}(\xi) \\
\text { tiled with at most } N \text { identical squares }
\end{array}\right)}{N^{d}},
\end{aligned}
$$

where $d=\operatorname{dim}_{\mathbb{C}} \mathcal{Q}(\xi)$. This convention for the normalization of the volume form on $\mathcal{Q}(\xi)$ follows the one in [AEZ1, AEZ2] and [G1, G2].

Given a quadratic differential $q$ on $X$ that is not the square of an Abelian differential, there is a unique (possibly ramified) connected double cover $p$ : $\hat{X} \rightarrow X$ such that the pull-back $p^{*} q$ is the square of an Abelian differential $p^{*} q=\omega^{2}$ globally defined on $\hat{X}$. The branch points of $p$ are the poles and the zeros of odd order of $q$. By convention, throughout Section 4, we always mark preimages of simple poles of $q$ on $\hat{X}$, and an alternative convention is considered in Appendix A. 
Note that for any $q$ in the same connected component $\mathcal{Q}(\xi)^{\text {comp }}$ of any stratum of meromorphic quadratic differentials with at most simple poles, the resulting Abelian differential $\omega$ belongs to the same connected component $\mathcal{H}(\kappa)^{\text {comp }}$ of the corresponding stratum of Abelian differentials. However, the correspondence $(X, q) \rightarrow(X, \omega)$ does not define a map from $\mathcal{Q}(\xi)^{\text {comp }}$ to $\mathcal{H}(\kappa)$ for there is an ambiguity in labeling zeros of $\omega$ as soon as $q$ has zeros of even order. Considering all possible ways to label the zeros of $\omega=p^{*} q$ for all $(X, q) \in \mathcal{Q}(\xi)^{\text {comp }}$, we obtain a suborbifold $\widehat{\mathcal{Q}}(\xi)^{\text {comp }} \subset \mathcal{H}(\kappa)^{\text {comp }}$ in appropriate component of appropriate stratum of Abelian differentials together with a covering map $P: \widehat{\mathcal{Q}}(\xi)^{\text {comp }} \rightarrow \mathcal{Q}(\xi)^{\text {comp }}$. By construction, the degree of the cover $P$ is $\operatorname{deg}(P)=2^{\ell\left(\xi_{\text {even }}\right)}$, where $\ell\left(\xi_{\text {even }}\right)=\xi_{0}+\xi_{2}+\xi_{4}+\cdots$ is the total number of marked points and of zeros of even degrees.

When $\widehat{\mathcal{Q}}(\xi)^{\text {comp }}$ contains several connected components, all components are pairwise isomorphic and all components form invariant arithmetic orbifolds. From now on, we choose any of these invariant arithmetic orbifolds $\mathcal{L}$.

The following result relates geometry of a connected component $\mathcal{Q}(\xi)^{\text {comp }}$ of a stratum of meromorphic quadratic differentials with at most simple poles and geometry of the induced invariant arithmetic orbifold $\mathcal{L}$.

Proposition 4.3. Let $\mathcal{Q}(\xi)^{\text {comp }}$ be a connected component of a stratum of meromorphic quadratic differentials with at most simple poles. Let $\mathcal{L}$ be the invariant arithmetic orbifold obtained by the canonical double cover (with marked preimages of simple poles, if any). Let $P: \mathcal{L} \rightarrow \mathcal{Q}(\xi)^{\text {comp }}$ be the natural cover. Then we have the following:

(1) Any square-tiled surface $(\hat{X}, \omega)$ in $\mathcal{L}$ always has an even number of squares.

(2) The natural involution $\hat{X} \rightarrow \hat{X}$ interchanging the sheets of the double cover $p: \hat{X} \rightarrow X$ sends squares of the tiling to squares of the tiling and does not map any square of the tiling to itself. In particular, the image under the map $P$ of any square-tiled surface $(\hat{X}, \omega)$ in $\mathcal{L}$ tiled with $2 N$ identical squares is a square-tiled surface $(X, q)$ in $\mathcal{Q}(\xi)^{\text {comp }}$ tiled with $N$ identical squares of the same size as the initial ones.

(3) The Masur-Veech volume element $d \mathrm{Vol}^{\mathcal{Q}(\xi)}$ on $\mathcal{Q}(\xi)^{\text {comp }}$ corresponding to normalization (4.6) and the Masur-Veech volume element $d \mathrm{Vol}^{\mathcal{L}}$ on $\mathcal{L}$ corresponding to normalization (4.5) are pointwise proportional:

$$
P^{*}\left(d \mathrm{Vol}^{\mathcal{Q}(\xi)}\right)=4^{d} \cdot d \mathrm{Vol}^{\mathcal{L}},
$$

where $d=\operatorname{dim}_{\mathbb{C}} \mathcal{Q}(\xi)$. In particular,

$$
\operatorname{Vol}_{1}^{\mathcal{L}}(\mathcal{L})=\frac{\operatorname{deg}(P)}{4^{d}} \cdot \operatorname{Vol}_{1}^{\mathcal{Q}(\xi)}\left(\mathcal{Q}_{1}(\xi)^{\operatorname{comp}}\right) .
$$


(4) If, furthermore, $\mathcal{Q}(\xi)=\mathcal{Q}\left(v,-1^{|v|+4}\right)$ is a stratum in genus 0 , then the cover $\widehat{\mathcal{Q}}\left(\nu,-1^{|v|+4}\right)=\mathcal{L}$ is connected and $\operatorname{deg}(P)=2^{\ell\left(\xi_{\text {even }}\right)}$, where $\ell\left(\xi_{\text {even }}\right)=$ $\xi_{0}+\xi_{2}+\xi_{4}+\cdots$ is the total number of marked points and of zeros of even degrees.

Proof. We postpone the Proof of Proposition 4.3 to Appendix A with an exception for the computation of proportionality coefficients which is performed below.

The factor $4^{d}$ relating the volume elements $P^{*}\left(d \mathrm{Vol}^{\mathcal{Q}(\xi)}\right)$ and $d \mathrm{Vol}^{\mathcal{L}}$ comes from the fact that, by convention, square-tiled surfaces in $\mathcal{Q}(\xi)$ are tiled with squares of size $\frac{1}{2} \times \frac{1}{2}$, while square-tiled surfaces in $\mathcal{L}$ are tiled with unit squares.

The cover $P: \widehat{\mathcal{Q}}(\xi)^{\text {comp }} \rightarrow \mathcal{Q}(\xi)^{\text {comp }}$ associates $\operatorname{deg}(P)=2^{\ell\left(\xi_{\text {even }}\right)}$ squaretiled surfaces in $\widehat{\mathcal{Q}}(\xi)^{\text {comp }}$ each tiled with $2 N$ identical squares to every squaretiled surface tiled with $N$ identical squares in $\mathcal{Q}(\xi)^{\text {comp }}$, and, reciprocally, any square-tiled surface in $\widehat{\mathcal{Q}}(\xi)^{\text {comp }}$ projects to a square-tiled surface in $\mathcal{Q}(\xi)^{\text {comp }}$. Comparing definitions (4.5) and (4.6), we get (4.7).

The proposition above allows us to treat any connected component of a stratum in the moduli space of meromorphic quadratic differentials with at most simple poles as a particular case of an invariant arithmetic orbifold.

4.4. Densities and uniform densities. Given an invariant arithmetic orbifold $\mathcal{L}$, we denote by $\mathcal{L}_{\mathbb{Z}}$ the set of square-tiled surfaces in $\mathcal{L}$. When the size of the square is not explicitly specified, we always assume that square-tiled surfaces are tiled with unit squares. In particular, $\mathcal{L}_{\mathbb{Z}}$ is the set of 'unit-square-tiled' surfaces, where we impose all standard assumptions on the tiling. Given a subset $\mathcal{D}_{\mathbb{Z}}$ of $\mathcal{L}_{\mathbb{Z}}$ and a subset $V$ of $\mathcal{L}$, define the following counting function:

$$
\mathcal{N}_{\mathcal{D}_{\mathbb{Z}}}(V, N):=\operatorname{Card}\left\{V \cap \mathcal{D}_{\mathbb{Z}}: \operatorname{Area}(S) \leqslant N\right\}=\operatorname{Card}\left\{V \cap \mathcal{D}_{\mathbb{Z}} \cap C_{\sqrt{N}} \mathcal{L}_{1}\right\} .
$$

Recall from Section 4.1 that the Masur-Veech volume $\operatorname{Vol}_{1}\left(\mathcal{L}_{1}\right)$ is finite and is defined as the leading term of the asymptotic of $\mathcal{N}_{\mathcal{L}_{\mathbb{Z}}}(\mathcal{L}, N)$ as $N \rightarrow+\infty$ normalized by a dimensional factor as

$$
\mathcal{N}_{\mathcal{L}_{\mathbb{Z}}}(\mathcal{L}, N)=\frac{1}{2 d} \cdot \operatorname{Vol}_{1}\left(\mathcal{L}_{1}\right) \cdot N^{d}+o\left(N^{d}\right) \quad \text { as } N \rightarrow+\infty,
$$

where $d=\operatorname{dim}_{\mathbb{C}} \mathcal{L}($ see $(4.5))$.

Definition 4.4. Let $\mathcal{L} \subset \mathcal{H}(\kappa)$ be an invariant arithmetic orbifold. We say that a subset $\mathcal{D}_{\mathbb{Z}} \subset \mathcal{L}_{\mathbb{Z}}$ has a density or, equivalently, is a density subset, if the 
following limit exists:

$$
\delta\left(\mathcal{D}_{\mathbb{Z}}\right):=\lim _{N \rightarrow+\infty} \frac{\mathcal{N}_{\mathcal{D}_{\mathbb{Z}}}(\mathcal{L}, N)}{\mathcal{N}_{\mathcal{L}_{\mathbb{Z}}}(\mathcal{L}, N)} .
$$

The value $\delta\left(\mathcal{D}_{\mathbb{Z}}\right)$ of the limit is called the density of the subset $\mathcal{D}_{\mathbb{Z}}$.

As two extreme examples, we have $\delta\left(\mathcal{L}_{\mathbb{Z}}\right)=1$ and $\delta(\varnothing)=0$. As another example, one can consider a sublattice of $\mathcal{L}_{\mathbb{Z}}$ in the period coordinates (see Section 4.1). The density of such sublattice is $\frac{1}{K}$ where $K$ is the index in $\mathcal{L}_{\mathbb{Z}}$ of the sublattice.

Note that a subset $\mathcal{D}_{\mathbb{Z}}$ of $\mathcal{L}_{\mathbb{Z}}$ has a density $\delta\left(\mathcal{D}_{\mathbb{Z}}\right)$ if and only if

$$
\mathcal{N}_{\mathcal{D}_{\mathbb{Z}}}(\mathcal{L}, N) \sim \delta\left(\mathcal{D}_{\mathbb{Z}}\right) \cdot \frac{\operatorname{Vol}_{1}\left(\mathcal{L}_{1}\right)}{2 d} \cdot N^{d},
$$

where equivalence is understood in the sense of equation (4.9). It is convenient to introduce the following quantity:

$$
c\left(\mathcal{D}_{\mathbb{Z}}\right):=\delta\left(\mathcal{D}_{\mathbb{Z}}\right) \cdot \operatorname{Vol}_{1}\left(\mathcal{L}_{1}\right) .
$$

We now introduce finer counting functions related to equidistribution. Recall that a cone in $\mathcal{L}$ is a subset of $\mathcal{L}$ preserved by dilation $S \mapsto \lambda S$; see (4.2).

Definition 4.5. Let $\mathcal{L} \subset \mathcal{H}(\kappa)$ be an invariant arithmetic orbifold. We say that a subset $\mathcal{D}_{\mathbb{Z}} \subset \mathcal{L}_{\mathbb{Z}}$ has a uniform density if for any open cone $C$ such that $\operatorname{Vol}_{1}\left(\partial C \cap \mathcal{L}_{1}\right)=0$, we have

$$
\lim _{N \rightarrow+\infty} \frac{\mathcal{N}_{\mathcal{D}_{\mathbb{Z}}}(C, N)}{\mathcal{N}_{\mathcal{L}_{\mathbb{Z}}}(C, N)}=\delta\left(\mathcal{D}_{\mathbb{Z}}\right) .
$$

Taking $C=\mathcal{L}$, we see that a uniform density is a density. The set $\mathcal{L}_{\mathbb{Z}}$ has uniform density. Indeed, it follows from zero measure boundary condition $\operatorname{Vol}_{1}\left(\partial C \cap \mathcal{L}_{1}\right)=0$ that the measure of $C \cap \mathcal{L}_{1}$ coincides with the leading coefficient in the integer point count (4.13). See also Remark 4.7.

The proposition below suggests several equivalent definitions of uniform density. In particular, it shows that uniformity of density of $\mathcal{D}_{\mathbb{Z}}$ is equivalent to equidistribution of $\mathcal{D}_{\mathbb{Z}}$ in $\mathcal{L}$ (see property (4)) and that uniformity of density of $\mathcal{D}_{\mathbb{Z}}$ in $\mathcal{L}$ is equivalent to uniformity of the projection of $\mathcal{D}_{\mathbb{Z}}$ to $\mathcal{L}_{1}$ (see property (2)).

Proposition 4.6. Let $\mathcal{L} \subset \mathcal{H}(\kappa)$ be an invariant arithmetic orbifold in some stratum of Abelian differentials. Let $\mathcal{D}_{\mathbb{Z}}$ be a subset of the set $\mathcal{L}_{\mathbb{Z}}$ of square-tiled surfaces in $\mathcal{L}$. Then the following assertions are equivalent: 
(1) $\mathcal{D}_{\mathbb{Z}}$ has uniform density.

(2) For any open set $V_{1} \subset \mathcal{L}_{1}$ with $\operatorname{Vol}_{1}\left(\partial V_{1}\right)=0$, one has

$$
\lim _{\varepsilon \rightarrow 0^{+}} \varepsilon^{2 d} \operatorname{Card}\left\{S \in \mathcal{D}_{\mathbb{Z}}: \varepsilon S \in C_{1} V_{1}\right\}=\frac{\delta\left(\mathcal{D}_{\mathbb{Z}}\right)}{2 d} \cdot \operatorname{Vol}_{1}\left(V_{1}\right) .
$$

(3) For any bounded nonnegative continuous function $f: \mathcal{L}_{1} \rightarrow \mathbb{R}_{+}$, one has

$$
\lim _{N \rightarrow+\infty} \frac{1}{N^{d}} \sum_{\substack{S \in \mathcal{D}_{\mathbb{Z}} \\ \operatorname{Area}(S) \leqslant N}} f\left(\frac{S}{\sqrt{\operatorname{Area}(S)}}\right)=\frac{\delta\left(\mathcal{D}_{\mathbb{Z}}\right)}{2 d} \cdot \int_{\mathcal{L}_{1}} f d \mathrm{Vol}_{1} .
$$

(4) For any open relatively compact set $V \subset \mathcal{L}$ with $\operatorname{Vol}(\partial V)=0$, one has

$$
\lim _{\varepsilon \rightarrow 0^{+}} \varepsilon^{2 d} \operatorname{Card}\left\{S \in \mathcal{D}_{\mathbb{Z}}: \varepsilon S \in V\right\}=\delta\left(\mathcal{D}_{\mathbb{Z}}\right) \cdot \operatorname{Vol}(V) .
$$

(5) For any compactly supported nonnegative continuous function $f: \mathcal{L} \rightarrow$ $\mathbb{R}_{+}$one has

$$
\lim _{\varepsilon \rightarrow 0^{+}} \varepsilon^{2 d} \sum_{S \in \mathcal{D}_{\mathbb{Z}}} f(\varepsilon S)=\delta\left(\mathcal{D}_{\mathbb{Z}}\right) \int_{\mathcal{L}} f d \mathrm{Vol}
$$

REMARK 4.7. One of the equivalent ways to define the Riemann integral consists in rescaling the mesh of integral points to approximate measure of the sets or integrals of functions. As a consequence, the above proposition remains true if one replaces a continuous function by a Riemann integrable function in assertions (3) and (5). Similarly, open sets with zero measure boundaries can be replaced by Jordan measurable sets in assertions (2) and (4). Recall that a set $V$ is called Jordan measurable if it is measurable and $\operatorname{Vol}(\stackrel{\circ}{V})=\operatorname{Vol}(\bar{V})$. It is a standard fact in integration that a set is Jordan measurable if and only if the characteristic function of this set is Riemann integrable.

Proof. Any open cone $C \subset \mathcal{L}$ can be realized as $C=C_{\infty} V_{1}$ for $V_{1}=C \cap \mathcal{L}_{1}$, see Definition (4.2), and, conversely, any open set $V_{1} \subset \mathcal{L}_{1}$ is the intersection of the open cone $C_{\infty} V_{1}$ with $\mathcal{L}_{1}$. Thus, similarly to (4.11), a subset $\mathcal{D}_{\mathbb{Z}}$ of $\mathcal{L}_{\mathbb{Z}}$ has a uniform density $\delta\left(\mathcal{D}_{\mathbb{Z}}\right)$ if and only if for every open set $V_{1} \subset \mathcal{L}_{1}$, one has

$$
\mathcal{N}_{\mathcal{D}_{\mathbb{Z}}}\left(C_{\infty} V_{1}, N\right) \sim \delta\left(\mathcal{D}_{\mathbb{Z}}\right) \cdot \frac{\operatorname{Vol}_{1}\left(V_{1}\right)}{2 d} \cdot N^{d} .
$$

Having a subset $V \subset \mathcal{L}$, define the quantity $\tilde{\mathcal{N}}_{\mathcal{D}_{\mathbb{Z}}}(V, \varepsilon):=\operatorname{Card}\left\{S \in \mathcal{D}_{\mathbb{Z}}: \varepsilon S \in\right.$ $V\}$. We can rewrite the left-hand side of the above asymptotic relation as 

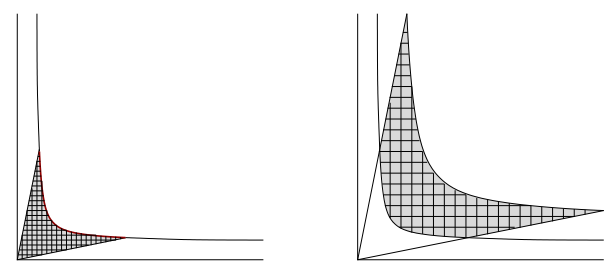

Figure 5. Cone based on the 'unit hyperboloid' on the left and part of the cone confined between two 'hyperboloids' on the right.

$$
\begin{aligned}
\mathcal{N}_{\mathcal{D}_{\mathbb{Z}}}\left(C_{\infty} V_{1}, N\right)= & \operatorname{Card}\left\{S \in \mathcal{D}_{\mathbb{Z}} \cap C_{\sqrt{N}} V_{1}\right\} \\
& =\operatorname{Card}\left\{S \in \frac{1}{\sqrt{N}} \mathcal{D}_{\mathbb{Z}} \cap C_{1} V_{1}\right\}=\tilde{\mathcal{N}}_{\mathcal{D}_{\mathbb{Z}}}\left(C_{1} V_{1}, \frac{1}{\sqrt{N}}\right) .
\end{aligned}
$$

We conclude that assertion (1) is equivalent to the following assertion:

$$
\lim _{N \rightarrow+\infty}\left(\frac{1}{\sqrt{N}}\right)^{2 d} \operatorname{Card}\left\{S \in \mathcal{D}_{\mathbb{Z}}:\left(\frac{1}{\sqrt{N}}\right) \cdot S \in C_{1} V_{1}\right\}=\frac{\delta\left(\mathcal{D}_{\mathbb{Z}}\right)}{2 d} \cdot \operatorname{Vol}_{1}\left(V_{1}\right) .
$$

It is clear that existence of the above limit for the discrete parameter $\varepsilon=\frac{1}{N}$ with $N \in \mathbb{N}$ and for the continuous parameter $\varepsilon \in \mathbb{R}_{+}$are equivalent. This proves that the first two assertions are equivalent, $(1) \Leftrightarrow(2)$.

By Definition (4.4) of the Masur-Veech volume, we have $\frac{1}{2 d} \operatorname{Vol}_{1}\left(V_{1}\right)=$ $\operatorname{Vol}\left(C_{1} V_{1}\right)$. This implies that assertion (2) is a particular case of assertion (4) when $V=C_{1} V_{1}$; so $(2) \Leftarrow(4)$.

We now prove the converse implication (2) $\Rightarrow(4)$. Assertion (2) implies assertion (4) when $V$ has the form of a cone $V=C_{1} V_{1}$ based on an open subset of the unit hyperboloid $\mathcal{L}_{1}$ (see the left picture in Figure 5). By homogeneity, this implies assertion (4) for any cone $C_{r} V_{1}$ with any $r>0$. Hence, it is also valid for any complement $C_{R} V_{1}-C_{r} V_{1}$ for any $R>r>0$ (see the right picture in Figure 5). Now, for any open relatively compact set $V$ in $\mathcal{L}$ and any $v>0$, one can find a finite disjoint collection of trapezoids $V^{(i)}=C_{R_{i}} V_{1}^{(i)}-C_{r} V_{1}^{(i)}$ contained in $V$ and such that the difference $V^{\text {dif }}$ between the union of trapezoids and $V$ has Masur-Veech measure less than $v$. As $v$ can be chosen arbitrarily small and as

$$
\begin{aligned}
0 & \leqslant \limsup _{\varepsilon \rightarrow 0^{+}} \varepsilon^{2 d} \operatorname{Card}\left\{S \in \mathcal{D}_{\mathbb{Z}}: \varepsilon S \in V\right\} \\
& \leqslant \lim _{\varepsilon \rightarrow 0^{+}} \varepsilon^{2 d} \operatorname{Card}\left\{S \in \mathcal{L}_{\mathbb{Z}}: \varepsilon S \in V\right\}=\operatorname{Vol}\left(V^{\mathrm{dif}}\right) \leqslant v,
\end{aligned}
$$

we conclude that assertion (2) implies assertion (4). We have proved equivalence (2) $\Leftrightarrow(4)$. 
Equivalence of assertions (2) and (3) (respectively of assertions (4) and (5)) follows directly from the duality of measures seen as linear forms on continuous functions.

4.5. Horocyclic invariance. Consider the following horocyclic subgroups of the group $\mathrm{SL}_{2}(\mathbb{Z})$ :

$$
\mathrm{U}_{h}(\mathbb{Z})=\left\{\left(\begin{array}{ll}
1 & n \\
0 & 1
\end{array}\right): n \in \mathbb{Z}\right\} \quad \text { and } \quad \mathrm{U}_{v}(\mathbb{Z})=\left\{\left(\begin{array}{ll}
1 & 0 \\
n & 1
\end{array}\right): n \in \mathbb{Z}\right\} .
$$

By definition, the $\mathrm{SL}_{2}(\mathbb{Z})$ action preserves the set of square-tiled surfaces. The aim of this section is to prove that for $\mathrm{U}_{h}(\mathbb{Z})$ or $\mathrm{U}_{v}(\mathbb{Z})$-invariant sets, a density is always uniform (see Definitions 4.4 and 4.5).

THEOREM 4.8. Let $\mathcal{L}$ be an invariant arithmetic orbifold and let $\mathcal{D}_{\mathbb{Z}}$ be a density subset of square-tiled surfaces in $\mathcal{L}$. If $\mathcal{D}_{\mathbb{Z}}$ is invariant under at least one of $\mathrm{U}_{h}(\mathbb{Z})$ or $\mathrm{U}_{v}(\mathbb{Z})$, then $\mathcal{D}_{\mathbb{Z}}$ has uniform density.

We start the Proof of Theorem 4.8 with the following preparatory lemma.

LEMMA 4.9. Any finite $\mathrm{SL}_{2}(\mathbb{R})$-invariant ergodic measure $v_{1}$ on any unit hyperboloid of a stratum of Abelian differentials is ergodic with respect to the actions of the discrete parabolic subgroups $\mathrm{U}_{h}(\mathbb{Z})$ and $\mathrm{U}_{v}(\mathbb{Z})$.

Proof. Let $\mathrm{G}$ be a simple Lie group, $\mathrm{H}$ be a closed noncompact subgroup of $\mathrm{G}$ and G-action be ergodic with respect to a finite invariant measure. By a particular case of Moore's Ergodicity theorem [Zim, Theorem 2.2.15], the H-action is also ergodic.

In our case, the simple Lie group is $\mathrm{SL}_{2}(\mathbb{R})$ and the closed noncompact subgroup $\mathrm{H}$ is $\mathrm{U}_{h}(\mathbb{Z})$.

REMARK 4.10. Note that in the general statement of Moore's Ergodic Theorem, the group $\mathrm{G}$ is a finite product of simple Lie groups with finite center, and the ergodic G-action is supposed to be irreducible (see [Zim, Theorem 2.2.15 and Definition 2.2.11]). However, for a simple Lie group $\mathrm{G}$, the requirement of irreducibility of the action is satisfied automatically; see the remark after [Zim, Definition 2.2.11].

Proof of Theorem 4.8. Let $\mathcal{L}_{1}$ be the intersection of $\mathcal{L}$ with the unit hyperboloid. The measure corresponding to the Masur-Veech volume element $d \mathrm{Vol}_{1}$ on $\mathcal{L}_{1}$ is ergodic with respect to the $\mathrm{SL}_{2}(\mathbb{R})$-action. 
Now, the subset $\mathcal{D}_{\mathbb{Z}}$ of $\mathcal{L}_{\mathbb{Z}}$ defines a sequence of discrete measures $\mu^{\left(N, \mathcal{D}_{\mathbb{Z}}\right)}$ on $\mathcal{L}_{1}$, where $N \in \mathbb{N}$. Namely, we put Dirac masses to all points represented by square-tiled surfaces tiled with at most $N$ unit squares which belong to $\mathcal{D}_{\mathbb{Z}}$. Then we project these points from $\mathcal{L}$ to $\mathcal{L}_{1}$ by the natural projection and normalize the resulting measure by $2 d \cdot N^{-d}$, where $d=\operatorname{dim}_{\mathbb{C}} \mathcal{L}$. We will show that for any bounded nonnegative continuous function $f: \mathcal{L}_{1} \rightarrow \mathbb{R}_{+}$, we have

$$
\lim _{N \rightarrow \infty} \int_{\mathcal{L}_{1}} f d \mu^{\left(N, \mathcal{D}_{\mathbb{Z}}\right)}=\delta\left(\mathcal{D}_{\mathbb{Z}}\right) \cdot \int_{\mathcal{L}_{1}} f d \mathrm{Vol}_{1} .
$$

Taking all square-tiled surfaces in $\mathcal{L}_{\mathbb{Z}}$, and not only those which belong to the subset $\mathcal{D}_{\mathbb{Z}}$, we get a sequence of measures which we denote by $\mu^{\left(N, \mathcal{L}_{\mathbb{Z}}\right)}$ and which weakly converges to our canonical invariant Masur-Veech measure $\mathrm{Vol}_{1}$ on $\mathcal{L}_{1}$; see (4.4). Recall that $\mathcal{L}_{1}$ is not compact; so by 'weak convergence', we mean that for any bounded nonnegative continuous function $f: \mathcal{L}_{1} \rightarrow \mathbb{R}_{+}$, we have

$$
\lim _{N \rightarrow \infty} \int_{\mathcal{L}_{1}} f d \mu^{\left(N, \mathcal{L}_{\mathbb{Z}}\right)}=\int_{\mathcal{L}_{1}} f d \mathrm{Vol}_{1}
$$

By definition, for any subset $\mathcal{D}_{\mathbb{Z}}$, we have

$$
\mu^{\left(N, \mathcal{D}_{\mathbb{Z}}\right)} \leqslant \mu^{\left(N, \mathcal{L}_{\mathbb{Z}}\right)}
$$

for we take only part of square-tiled surfaces of area at most $N$ to define $\mu^{\left(N, \mathcal{D}_{\mathbb{Z}}\right)}$, while we take all square-tiled surface of area at most $N$ to define $\mu^{\left(N, \mathcal{L}_{\mathbb{Z}}\right)}$. Since the normalization factor $2 d \cdot N^{-d}$ is the same in both cases, we get the desired inequality. This implies, in particular, that for any Jordan measurable subset $K \subset$ $\mathcal{L}_{1}$ with compact closure, one has

$$
\limsup _{N \rightarrow+\infty} \mu^{\left(N, \mathcal{D}_{\mathbb{Z}}\right)}(K) \leqslant \lim _{N \rightarrow+\infty} \mu^{\left(N, \mathcal{L}_{\mathbb{Z}}\right)}(K)=\operatorname{Vol}_{1}(K) .
$$

Therefore, any subsequence of $\left\{\mu^{\left(N, \mathcal{D}_{\mathbb{Z}}\right)}\right\}_{N \geqslant 0}$ contains a converging subsequence.

From now on, we fix a measure $\mu_{J}$ obtained as the weak limit of some subsequence $\left\{\mu^{\left(N_{k}, \mathcal{D}_{\mathbb{Z}}\right)}\right\}_{k \geqslant 0}$. Domination (4.15) and the fact that $\mu^{\left(N, \mathcal{L}_{\mathbb{Z}}\right)}$ converges to the Masur-Veech measure $\mathrm{Vol}_{1}$ implies that $\mu_{J}$ is absolutely continuous with respect to $\mathrm{Vol}_{1}$. Moreover, the $\mathrm{U}_{h}(\mathbb{Z})$-invariance of $\mathcal{D}_{\mathbb{Z}}$ implies that all measures $\mu^{\left(N_{k}, \mathcal{D}_{\mathbb{Z}}\right)}$ are $\mathrm{U}_{h}(\mathbb{Z})$-invariant. Hence, the weak limit $\mu_{J}$ is also $\mathrm{U}_{h}(\mathbb{Z})$-invariant.

By Lemma 4.9, the Masur-Veech measure $\mathrm{Vol}_{1}$ is ergodic with respect to the action of $\mathrm{U}_{h}(\mathbb{Z})$. Ergodicity of $\mathrm{Vol}_{1}$ with respect to the action of $\mathrm{U}_{h}(\mathbb{Z})$, invariance of $\mu_{J}$ under the action of $\mathrm{U}_{h}(\mathbb{Z})$ and the fact that $\mu_{J}$ is absolutely continuous with respect to $\mathrm{Vol}_{1}$ all together imply that the two measures are proportional:

$$
\mu_{J}=k_{J} \cdot \mathrm{Vol}_{1} .
$$


The coefficient of proportionality $k_{J}$ can be obtained by integrating the constant function 1. By Definition (4.10) of density $\delta\left(\mathcal{D}_{\mathbb{Z}}\right)$, we have

$$
k_{J}=\lim _{k \rightarrow \infty} \frac{\mu^{\left(N_{k}, \mathcal{D}_{\mathbb{Z}}\right)}\left(\mathcal{L}_{1}\right)}{\mu^{\left(N_{k}, \mathcal{L}_{\mathbb{Z}}\right)}\left(\mathcal{L}_{1}\right)}=\lim _{k \rightarrow \infty} \frac{\mathcal{N}_{\mathcal{D}_{\mathbb{Z}}}\left(\mathcal{L}, N_{k}\right)}{\mathcal{N}_{\mathcal{L}_{\mathbb{Z}}}\left(\mathcal{L}, N_{k}\right)}=\delta\left(\mathcal{D}_{\mathbb{Z}}\right)
$$

for any weak limit $\mu_{J}$. This implies (4.14), which correspond to assertion (3) in the list of equivalent definitions of uniform density of $\mathcal{D}_{\mathbb{Z}}$ given in Proposition 4.6. This concludes the Proof of Theorem 4.8.

REMARK 4.11. Our proof is inspired by the Proof of [Mi, Theorem 6.4] where (in the language of the current paper) Mirzakhani proves that ergodicity of the action of the mapping class group $\operatorname{Mod}_{g, n}$ with respect to the Thurston measure on the space of measured laminations $\mathcal{M L}_{g, n}$ implies uniform density of the $\operatorname{Mod}_{g, n}$-orbit of any integral multicurve with respect to the set of all integral multicurves $\mathcal{M} \mathcal{L}_{g, n}^{\mathbb{Z}}$.

REMARK 4.12. Theorem 4.8 can be easily illustrated in the context of the usual Lebesgue measure on $\mathbb{R}^{2}$. Let us consider the subset $\mathcal{D}_{\mathbb{Z}, 1}$ of those points of $\mathbb{Z}^{2}$ which have coprime coordinates. For any $R>0$, one can place a ball of radius $R$ in $\mathbb{R}^{2}$ in such a way that the ball would not contain a single point of $\mathcal{D}_{\mathbb{Z}, 1}$. In this aspect, $\mathcal{D}_{\mathbb{Z}, 1}$ does not resemble a sublattice of $\mathbb{Z}^{2}$. Nevertheless, $\mathcal{D}_{\mathbb{Z}, 1}$ is $\mathrm{SL}_{2}(\mathbb{Z})$-invariant, which implies that $\mathcal{D}_{\mathbb{Z}, 1}$ has uniform density $\delta\left(\mathcal{D}_{\mathbb{Z}, 1}\right)$. The value of $\delta\left(\mathcal{D}_{\mathbb{Z}, 1}\right)$ can be evaluated explicitly as $\delta\left(\mathcal{D}_{\mathbb{Z}, 1}\right)=\frac{6}{\pi^{2}}$. In particular, one can compute areas of Jordan measurable sets $V$ in $\mathbb{R}^{2}$ by counting the number of points of $\varepsilon \mathcal{D}_{\mathbb{Z}, 1}$ which get to $V$ and letting the mesh $\varepsilon$ of the grid tend to zero:

$$
\operatorname{Area}(V)=\frac{\pi^{2}}{6} \cdot \lim _{\varepsilon \rightarrow+0} \varepsilon^{2} \cdot \operatorname{Card}\left\{V \cap \varepsilon \mathcal{D}_{\mathbb{Z}, 1}\right\}
$$

For each $k \in \mathbb{N}$, consider the analogous subset $\mathcal{D}_{\mathbb{Z}, k} \subset \mathbb{Z}^{2}$ of points with integer coordinates $(x, y)$ such that $\operatorname{gcd}(x, y)=k$. By definition, $\mathcal{D}_{\mathbb{Z}, k}$ is obtained from $\mathcal{D}_{\mathbb{Z}, 1}$ by homothety with dilatation coefficient $k$. Thus, any subset $\mathcal{D}_{\mathbb{Z}, k}$ also has uniform density, and $\delta\left(\mathcal{D}_{\mathbb{Z}, k}\right)=\frac{1}{k^{2}} \cdot \delta\left(\mathcal{D}_{\mathbb{Z}, 1}\right)$. We have

$$
\mathbb{Z}^{2}=\bigsqcup_{k \in \mathbb{N}} \mathcal{D}_{\mathbb{Z}, k}
$$

which leads to

$$
1=\delta\left(\mathbb{Z}^{2}\right)=\sum_{k=1}^{+\infty} \delta\left(\mathcal{D}_{\mathbb{Z}, k}\right)=\sum_{k=1}^{+\infty} \frac{1}{k^{2}} \cdot \delta\left(\mathcal{D}_{\mathbb{Z}, 1}\right)=\zeta(2) \cdot \delta\left(\mathcal{D}_{\mathbb{Z}, 1}\right) .
$$


4.6. Invariance with respect to Re- and Im-foliations. Given two subsets $\mathcal{D}_{\mathbb{Z}}$ and $\mathcal{D}_{\mathbb{Z}}^{\prime}$ of square-tiled surfaces that admit (uniform) densities, it is, in general, false that $\delta\left(\mathcal{D}_{\mathbb{Z}} \cap \mathcal{D}_{\mathbb{Z}}^{\prime}\right)=\delta\left(\mathcal{D}_{\mathbb{Z}}\right) \cdot \delta\left(\mathcal{D}_{\mathbb{Z}}^{\prime}\right)$. We introduce in this section Reand Im-invariance that provides sufficient conditions for this equality to hold.

A stratum of Abelian differentials of complex dimension $d$ is endowed with a pair of transverse foliations of real dimension $d$ induced from the canonical direct sum decomposition in period coordinates

$$
H^{1}(S, \Sigma ; \mathbb{C})=H^{1}(S, \Sigma ; \mathbb{R}) \oplus H^{1}(S, \Sigma ; i \mathbb{R})
$$

In particular, in a neighborhood of any point $(X, \omega)$ of the stratum, one has canonical direct product structure in period coordinates. Locally, leaves of the Im-foliation are preimages of points under projection to the first summand, and leaves of the Re-foliation are projections to the second summand. In other words, pairs $(X, \omega)$ in a leaf of the Im-foliation (respectively of the Re-foliation) share the cohomology class $[\operatorname{Re} \omega] \in H^{1}(S, \Sigma ; \mathbb{R})\left(\right.$ respectively $[\operatorname{Im} \omega] \in H^{1}(S$, $\Sigma ; \mathbb{R})$ ).

By the results of Eskin-Mirzakhani-Mohammadi [EMi], [EMiMo1] (see the precise statement on page 28), the analogous decomposition holds for any $\mathrm{GL}_{2}(\mathbb{R})$-invariant suborbifold $\mathcal{L}$. If $\mathcal{L}$ is locally represented in period coordinates as a finite union of several linear subspaces, every such subspace is foliated by Re and Im-foliations. We formalize this trivial corollary of highly nontrivial results of Eskin-Mirzakhani-Mohammadi as a separate assertion.

Proposition 4.13. Let $\mathcal{L}$ be an invariant arithmetic suborbifold in some stratum $\mathcal{H}(\kappa)$ of Abelian differentials. Then the Re-leaf in $\mathcal{L}$ passing through a point $(X, \omega)$ of $\mathcal{L}$ coincides with the connected component of the intersection of the Re-leaf in the ambient stratum $\mathcal{H}(\kappa)$ passing through $(X, \omega)$ with $\mathcal{L}$.

In period coordinates $H^{1}(S, \Sigma ; \mathbb{C})$ in the neighborhood of $(X, \omega)$, the Releaf in $\mathcal{L}$ passing through a point $(X, \omega)$ is represented by a finite union of linear subspaces of real dimension $d=\operatorname{dim}_{\mathbb{C}} \mathcal{L}$.

DEFINITION 4.14. We say that a subset $\mathcal{D}_{\mathbb{Z}} \subset \mathcal{L}_{\mathbb{Z}}$ of square-tiled surfaces in an invariant arithmetic suborbifold $\mathcal{L}$ is Re-invariant (respectively Im-invariant) if for any point $S$ in $\mathcal{D}_{\mathbb{Z}}$ all points of $\mathcal{L}_{\mathbb{Z}}$ located in the leaf of the Re-foliation (respectively Im-foliation) in $\mathcal{L}$ passing through $S$ also belong to $\mathcal{D}_{\mathbb{Z}}$.

REMARK 4.15. In a very similar context in homogeneous dynamics, the analogous properties are called stable and unstable horocyclic invariance. 
Note that the unipotent subgroups

$$
\mathrm{U}_{h}(\mathbb{R})=\left\{\left(\begin{array}{ll}
1 & t \\
0 & 1
\end{array}\right): t \in \mathbb{R}\right\} \quad \text { and } \quad \mathrm{U}_{v}(\mathbb{R})=\left\{\left(\begin{array}{ll}
1 & 0 \\
t & 1
\end{array}\right): t \in \mathbb{R}\right\}
$$

act along the leaves of the Re-foliation and Im-foliation, respectively. Thus, any Re-invariant (respectively Im-invariant) subset $\mathcal{D}_{\mathbb{Z}}$ of square-tiled surface is automatically $\mathrm{U}_{h}(\mathbb{Z})$-invariant (respectively $\mathrm{U}_{v}(\mathbb{Z})$-invariant). The converse is obviously not true: the $\mathrm{U}_{h}(\mathbb{Z})$-orbit of any square-tiled surface of genus $g \geqslant 2$ is $\mathrm{U}_{h}(\mathbb{Z})$-invariant but usually not Re-invariant.

THEOREM 4.16. Let $\mathcal{D}_{\mathbb{Z}}^{h}, \mathcal{D}_{\mathbb{Z}}^{v}$ be subsets of square-tiled surfaces in an invariant arithmetic orbifold $\mathcal{L}$ that are, respectively, Re-invariant and $\mathrm{Im}$-invariant. Then $\mathcal{D}_{\mathbb{Z}}^{h}, \mathcal{D}_{\mathbb{Z}}^{v}$ and $\mathcal{D}_{\mathbb{Z}}^{h} \cap \mathcal{D}_{\mathbb{Z}}^{v}$ have uniform densities and the following equality holds:

$$
\delta\left(\mathcal{D}_{\mathbb{Z}}^{h} \cap \mathcal{D}_{\mathbb{Z}}^{v}\right)=\delta\left(\mathcal{D}_{\mathbb{Z}}^{h}\right) \cdot \delta\left(\mathcal{D}_{\mathbb{Z}}^{v}\right) .
$$

Proof. The uniformity of the density of $\mathcal{D}_{\mathbb{Z}}^{h}$ and of $\mathcal{D}_{\mathbb{Z}}^{v}$ follows from Theorem 4.8.

Following the Proof of Proposition 4.6, define the following quantity:

$$
\tilde{\mathcal{N}}_{\mathcal{D}_{\mathbb{Z}}}(V, \varepsilon)=\operatorname{Card}\left\{S \in \mathcal{D}_{\mathbb{Z}}: \varepsilon S \in V\right\} .
$$

We prove now assertion (4) from Proposition 4.6 for the set $\mathcal{D}_{\mathbb{Z}}^{h} \cap \mathcal{D}_{\mathbb{Z}}^{v}$. By Proposition 4.6, this assertion is equivalent to uniformity of the density of $\mathcal{D}_{\mathbb{Z}}^{h} \cap$ $\mathcal{D}_{\mathbb{Z}}^{v}$. It is sufficient to prove assertion (4) for sets $V$ of the form $I \times J$ where $I$ and $J$ are, respectively, Jordan measurable in the summands (4.16) in the natural product structure of $\mathcal{L}$ provided by $\mathrm{Re}$ and Im foliations (see Remark 4.7 for the definition of Jordan measurable). Indeed, any Jordan measurable set in $\mathcal{L}$ can be approximated from below and above by a finite union of products of Jordan measurable sets of the form $I \times J$ up to an arbitrary small difference in measures. We also assume that any $V$ is located in a single coordinate chart in period coordinates so that the products $p_{\mathrm{Re}}$ and $p_{\mathrm{Im}}$ to the first and the second terms of (4.16) are well defined, $I=p_{\mathrm{Re}}(V) ; J=p_{\mathrm{Im}}(V)$ and $V=I \times J$.

Re- and Im-invariance of $\mathcal{D}_{\mathbb{Z}}^{h}$ and $\mathcal{D}_{\mathbb{Z}}^{v}$, respectively, implies that the sets $\varepsilon \mathcal{D}_{\mathbb{Z}}^{h}$, $\varepsilon \mathcal{D}_{\mathbb{Z}}^{v}$ and $\varepsilon \cdot\left(\mathcal{D}_{\mathbb{Z}}^{h} \cap \mathcal{D}_{\mathbb{Z}}^{v}\right)$ locally have the following product structures:

$$
\begin{aligned}
\varepsilon \mathcal{D}_{\mathbb{Z}}^{h} \cap V & =\left(I \cap p_{\mathrm{Re}}\left(\varepsilon \mathcal{L}_{\mathbb{Z}}\right)\right) \times\left(J \cap p_{\mathrm{Im}}\left(\varepsilon \mathcal{D}_{\mathbb{Z}}^{h}\right)\right), \\
\varepsilon \mathcal{D}_{\mathbb{Z}}^{v} \cap V & =\left(I \cap p_{\operatorname{Re}}\left(\varepsilon \mathcal{D}_{\mathbb{Z}}^{v}\right)\right) \times\left(J \cap p_{\operatorname{Im}}\left(\varepsilon \mathcal{L}_{\mathbb{Z}}\right)\right), \\
\varepsilon \cdot\left(\mathcal{D}_{\mathbb{Z}}^{h} \cap \mathcal{D}_{\mathbb{Z}}^{v}\right) \cap V & =\left(I \cap p_{\operatorname{Re}}\left(\varepsilon \mathcal{D}_{\mathbb{Z}}^{v}\right)\right) \times\left(J \cap p_{\operatorname{Im}}\left(\varepsilon \mathcal{D}_{\mathbb{Z}}^{h}\right)\right),
\end{aligned}
$$

where

$$
I \cap p_{\operatorname{Re}}\left(\varepsilon \mathcal{L}_{\mathbb{Z}}\right)=I \cap H^{1}(S, \Sigma ; \varepsilon \mathbb{Z}),
$$




$$
J \cap p_{\operatorname{Im}}\left(\varepsilon \mathcal{L}_{\mathbb{Z}}\right)=J \cap H^{1}(S, \Sigma ; i \varepsilon \mathbb{Z}) .
$$

This implies the following relations for the counting functions:

$$
\begin{aligned}
\tilde{\mathcal{N}}_{\mathcal{L}_{\mathbb{Z}}}(V, \varepsilon) & =\operatorname{Card}\left(I \cap p_{\operatorname{Re}}\left(\varepsilon \mathcal{L}_{\mathbb{Z}}\right)\right) \cdot \operatorname{Card}\left(J \cap p_{\operatorname{Im}}\left(\varepsilon \mathcal{L}_{\mathbb{Z}}\right)\right), \\
\tilde{\mathcal{N}}_{\mathcal{D}_{\mathbb{Z}}^{h}}(V, \varepsilon) & =\operatorname{Card}\left(I \cap p_{\operatorname{Re}}\left(\varepsilon \mathcal{L}_{\mathbb{Z}}\right)\right) \cdot \operatorname{Card}\left(J \cap p_{\operatorname{Im}}\left(\varepsilon \mathcal{D}_{\mathbb{Z}}^{h}\right)\right), \\
\tilde{\mathcal{N}}_{\mathcal{D}_{\mathbb{Z}}^{v}}(V, \varepsilon) & =\operatorname{Card}\left(I \cap p_{\operatorname{Re}}\left(\varepsilon \mathcal{D}_{\mathbb{Z}}^{v}\right)\right) \cdot \operatorname{Card}\left(J \cap p_{\operatorname{Im}}\left(\varepsilon \mathcal{L}_{\mathbb{Z}}\right)\right), \\
\tilde{\mathcal{N}}_{\mathcal{D}^{h} \cap \mathcal{D}^{v}}(V, \varepsilon) & =\operatorname{Card}\left(I \cap p_{\operatorname{Re}}\left(\varepsilon \mathcal{D}_{\mathbb{Z}}^{v}\right)\right) \cdot \operatorname{Card}\left(J \cap p_{\operatorname{Im}}\left(\varepsilon \mathcal{D}_{\mathbb{Z}}^{h}\right)\right) .
\end{aligned}
$$

Thus, for any set $V=I \times J$ as above, we have the equality

$$
\frac{\tilde{\mathcal{N}}_{\mathcal{D}_{\mathbb{Z}}^{h} \cap \mathcal{D}_{\mathbb{Z}}^{v}}(V, \varepsilon)}{\tilde{\mathcal{N}}_{\mathcal{L}_{\mathbb{Z}}}(V, \varepsilon)}=\frac{\tilde{\mathcal{N}}_{\mathcal{D}_{\mathbb{Z}}^{h}}(V, \varepsilon)}{\tilde{\mathcal{N}}_{\mathcal{L}_{\mathbb{Z}}}(V, \varepsilon)} \cdot \frac{\tilde{\mathcal{N}}_{\mathcal{D}_{\mathbb{Z}}^{v}}(V, \varepsilon)}{\tilde{\mathcal{N}}_{\mathcal{L}_{\mathbb{Z}}}(V, \varepsilon)} .
$$

The definition of the Masur-Veech volume and the fact that $\mathcal{D}_{\mathbb{Z}}^{h}$ and $\mathcal{D}_{\mathbb{Z}}^{v}$ have uniform densities imply the existence of the limits:

$$
\begin{array}{r}
\lim _{\varepsilon \rightarrow+\infty} \frac{\tilde{\mathcal{N}}_{\mathcal{D}_{\mathbb{Z}}^{h}}(V, \varepsilon)}{\widetilde{\mathcal{N}}_{\mathcal{L}_{\mathbb{Z}}}(V, \varepsilon)}=\delta\left(\mathcal{D}_{\mathbb{Z}}^{h}\right), \\
\lim _{\varepsilon \rightarrow+\infty} \frac{\tilde{\mathcal{N}}_{\mathcal{D}_{\mathbb{Z}}^{v}}(V, \varepsilon)}{\tilde{\mathcal{N}}_{\mathcal{L}_{\mathbb{Z}}}(V, \varepsilon)}=\delta\left(\mathcal{D}_{\mathbb{Z}}^{v}\right) .
\end{array}
$$

Hence,

$$
\lim _{\varepsilon \rightarrow+\infty} \frac{\tilde{\mathcal{N}}_{\mathcal{D}_{\mathbb{Z}}^{h} \cap \mathcal{D}_{\mathbb{Z}}^{v}}(V, \varepsilon)}{\widetilde{\mathcal{N}}_{\mathcal{L}_{\mathbb{Z}}}(V, \varepsilon)}=\delta\left(\mathcal{D}_{\mathbb{Z}}^{h}\right) \cdot \delta\left(\mathcal{D}_{\mathbb{Z}}^{v}\right),
$$

which is equivalent to analogous relation written in the form of the assertion (4) from Proposition 4.6.

4.7. Separatrix diagrams and critical graphs. In this section, we recall some basic facts concerning combinatorial geometry of Jenkins-Strebel differentials. Assume that all leaves of the horizontal foliation of an Abelian or quadratic differential are either closed or connect critical points (a leaf joining two critical points and having no critical points in its interior is called a saddle connection or a separatrix). An Abelian or quadratic differential having this property is called a Jenkins-Strebel differential; see [Str]. For example, square-tiled surfaces provide particular cases of Jenkins-Strebel differentials.

Following [KoZo], we associate to each Jenkins-Strebel differential a combinatorial data called a separatrix diagram (or critical graph in terminology 
of [DdHb]). A separatrix diagram $\Gamma$ encodes the combinatorial geometry of the completely periodic horizontal foliation. It is a ribbon graph composed of a tubular neighborhood of the union of all horizontal saddle connections. This ribbon graph is endowed with the partition of boundary components into pairs, where two components are paired when they are joined by a cylinder filled with regular periodic horizontal leaves.

LEMMA 4.17. A separatrix diagram representing a Jenkins-Strebel Abelian differential in a stratum of complex dimension $d$ has exactly $d-1$ edge (that is, $d-1$ horizontal saddle connections).

A separatrix diagram representing a meromorphic Jenkins-Strebel quadratic differential with at most simple poles in a stratum of complex dimension d has exactly d edges (that is, $d$ horizontal saddle connections).

Proof. A critical point corresponding to a zero of an Abelian differential of degree $k$ has $k+1$ incoming and $k+1$ outgoing separatrix rays, where a marked point is interpreted in this context as a 'zero of degree 0 '. Thus, the total number $n$ of horizontal saddle connections satisfies

$$
n=\text { sum of degrees of zeros }+ \text { (number of zeros and marked points). }
$$

Note that

$$
2 g-2=\text { sum of degrees of zeros }
$$

and that

$$
d=2 g+\text { (number of zeros and marked points) }-1,
$$

which implies the equality $n=d-1$.

Similarly, a critical point corresponding to a zero (or to a simple pole) of degree $k$ of a quadratic differential has $k+2$ horizontal separatrix rays, where a simple pole is interpreted in this context as a 'zero of degree -1 '. Thus, the total number of horizontal saddle connections of a Jenkins-Strebel quadratic differential equals half the total number of separatrix rays. On the other hand, the sum of degrees of zeros and simple poles equals $4 g-4$ and the dimension of the stratum equals

$$
d=2 g+\text { (number of zeros, marked points and simple poles })-2,
$$

which implies the assertion for quadratic differentials.

Removing all horizontal saddle connections of an Abelian Jenkins-Strebel differential from the associated translation surface, we decompose the surface into a disjoint union of maximal cylinders filled with closed regular leaves of 
the horizontal foliation. Denote by $m$ the number of these maximal horizontal cylinders. The corresponding separatrix diagram viewed as a ribbon graph has $2 m$ boundary components.

We will say that a vector $v \in \mathbb{R}^{n}$ is strictly positive if all its components are strictly positive numbers, that is, if $v$ belongs to $\mathbb{R}_{+}^{n}=\mathbb{R}_{>0}^{n}$. Any Jenkins-Strebel differential having a given separatrix diagram $\Gamma$ is completely determined by the following additional length data:

- the length of each horizontal saddle connection $\left(\ell_{i}\right)_{i \in\{1, \ldots, d-1\}} \in \mathbb{R}_{>0}^{d-1}$,

- the height of each maximal horizontal cylinder $\left(h_{j}\right)_{j \in\{1, \ldots, m\}} \in \mathbb{R}_{>0}^{m}$,

- a twist parameter for each cylinder $\left(t_{j}\right)_{j \in\{1, \ldots, m\}} \in \mathbb{R}^{m}$. The twist parameter $t_{j}$ is determined by a choice of a saddle connection $\gamma_{j}$ entirely contained in the corresponding cylinder and joining the two opposite boundary components of this cylinder.

For an Abelian Jenkins-Strebel differential $\omega$, the length $\ell_{i}$ of each horizontal saddle connection $\lambda_{i}$ coincides with the period $\int_{\lambda_{i}} \omega$ under appropriate choice of orientation of $\lambda_{i}$. Under appropriate choice of orientation of $\gamma_{j}$, the period of $\omega$ along the saddle connection $\gamma_{j}$ equals $t_{j}+i \cdot h_{j}$, where $i=\sqrt{-1}$ in the latter expression.

In the example presented in Figure 6, all horizontal saddle connections of the separatrix diagram corresponding to the Abelian differential $\omega$ have lengths $\ell_{i}=$ $\left|\lambda_{i}\right|=1$ for $i=1, \ldots, 8$. The height $h_{1}$ of the single maximal horizontal cylinder is equal to 1 and the twist parameter corresponding to the saddle connection $\gamma_{1}$ (represented by the lateral sides of the parallelogram pattern) is also equal to 1 . The relative period $\int_{\gamma_{1}} \omega$ is equal to $1+i$, where $i=\sqrt{-1}$ in the latter expression.

Similarly, for a Jenkins-Strebel quadratic differential $q$ on a complex curve $X$, the length parameters $(\ell, t, h)$ can be interpreted in terms of periods of the holomorphic form $\omega$ on the canonical double cover $p: \hat{X} \rightarrow X$ where $p^{*} q=\omega^{2}$.

The length data is subject to the constraint that the sum of the lengths of saddle connections on respectively top and bottom boundary components of each maximal horizontal cylinder should be the same. We denote by $w_{\Gamma, j}^{\text {top }}(\ell)$ (respectively by $w_{\Gamma, j}^{\text {bot }}(\ell)$ ) the sum of the lengths $\ell_{i}$ of those horizontal saddle connections that appear on the top (respectively bottom) of the $j$ th cylinder. Note that in the case of quadratic differentials, the same saddle connection might appear at the same boundary component twice. In this case, the corresponding parameter $\ell_{i}$ is counted with weight 2 . The linear conditions are then expressed as

$$
w_{\Gamma, j}^{\text {top }}(\ell)=w_{\Gamma, j}^{\text {bot }}(\ell) \quad \text { for } j=1, \ldots, m
$$




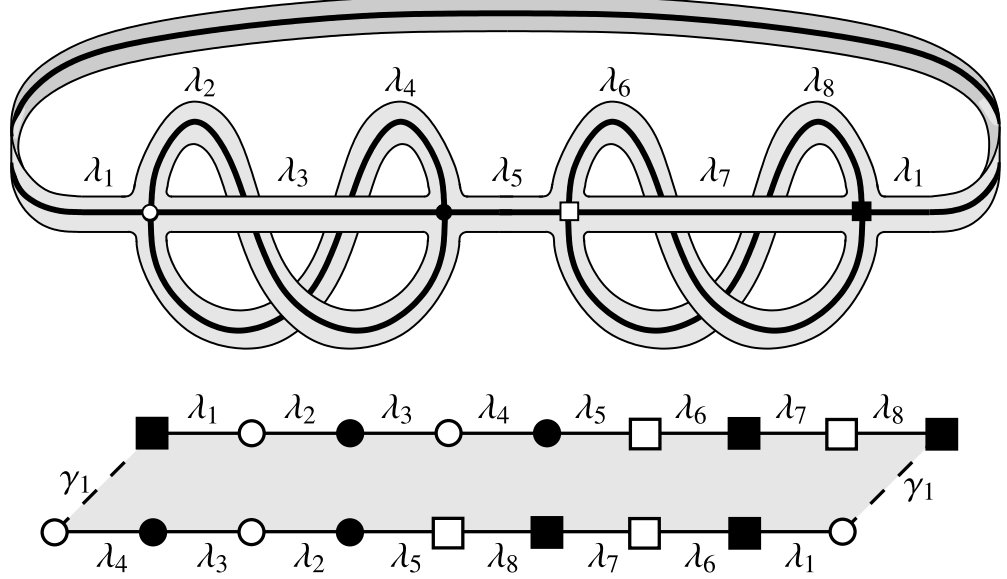

Figure 6. A Jenkins-Strebel Abelian differential with a single cylinder represented as a ribbon graph (on top) and as a parallelogram on the bottom. This Abelian differential belongs to the stratum $\mathcal{H}\left(1^{4}\right)$ of complex dimension $d=9$ and has $9-1=8$ horizontal saddle connections.

For an Abelian Jenkins-Strebel differential, there is an obvious linear relation between these constraints. Namely, since every horizontal saddle connection is present exactly once on top of some cylinder and exactly once on the bottom of some cylinder, taking the sum of equations (4.17), we get a tautological identity $\sum_{j=1}^{m} w_{\Gamma, j}^{\text {top }}=\sum_{j=1}^{m} w_{\Gamma, j}^{\text {bot }}$ having the sum $\ell_{1}+\ell_{2}+\cdots+\ell_{d-1}$ (arranged in a certain order) on each side of the identity. Lemma 4.18 proves that this is the only relation between equations (4.17) for Abelian differentials and that equations (4.17) are independent for quadratic differentials.

LEMMA 4.18. Consider the decomposition of a Jenkins-Strebel Abelian differential or of a Jenkins-Strebel quadratic differential with at most simple poles into maximal horizontal cylinders obtained by removing from the surface all horizontal saddle connections. Let $m$ be the number of such cylinders.

The linear system (4.17) has rank $m-1$ for Abelian Jenkins-Strebel differentials and rank $m$ for quadratic Jenkins-Strebel differentials.

Proof. We start with the case of Abelian Jenkins-Strebel differentials. Let $r$ be the rank of linear system (4.17). We have seen that the length parameters $\ell_{i}, t_{j}$ can be interpreted as cocycles in the relative cohomology group $H^{1}(S, \Sigma, \mathbb{R})$. The cylinder twists $t_{j}$ can be chosen arbitrarily, which gives $m$ free parameters 
independent of parameters $\ell_{i}$. We can complete them with $(d-1)-r$ additional free parameters $\ell_{i}$. Since $\operatorname{dim}_{\mathbb{R}} H^{1}(S, \Sigma, \mathbb{R})=d$, we conclude that $r \geqslant m-1$. On the other hand, we have already seen that $r \leqslant m-1$ since there is at least one linear relation between equations (4.17).

The count for quadratic Jenkins-Strebel differentials is analogous. The $m$ twists $t_{j}$ provide $m$ independent parameters. However, this time by Lemma 4.17, we have $d$ horizontal saddle connections, and the linear span of all independent parameters $\ell_{i}, t_{j}$ can have real dimension at most $d$, which implies that linear system (4.17) has to have the full rank $r=m$.

We will use the following simple lemma in the Proof of Proposition 4.20.

Lemma 4.19. Let $(X, \omega)$ be a Jenkins-Strebel holomorphic 1-form on a complex curve $X$. Consider the decomposition of $X$ into union of maximal cylinders filled with closed horizontal leaves. Consider the collection of all horizontal saddle connections completed for each cylinder by a nonhorizontal segment inside the cylinder joining some pair of singularities on the two boundary components of the cylinder. Viewed as a collection of relative homology cycles, such a collection of saddle connections spans the entire relative homology group $H_{1}(X, \Sigma ; \mathbb{Z})$.

Proof. The complement to the union of our segments is a disjoint union of topological discs. Thus, the collection of segments as above defines a 1-skeleton of a $C W$ decomposition of the topological surface $S$ underlying the complex curve $X$, where all 0 -cells belong to the finite set $\Sigma$ of zeros and marked points of the differential.

The following simple observation is of crucial importance for us.

Proposition 4.20. Let $\Gamma$ be the separatrix diagram corresponding to a Jenkins-Strebel Abelian differential $(X, \omega)$. Denote by $h$ the vector of heights of the associated maximal horizontal cylinders. Let $\mathcal{H}(\kappa)^{\mathrm{comp}}$ be the connected component of the ambient stratum containing $(X, \omega)$. Consider the subset $\mathcal{H}(\kappa)_{\Gamma, h}^{\text {comp }} \subset \mathcal{H}(\kappa)^{\text {comp }}$ of all Jenkins-Strebel differentials in $\mathcal{H}(\kappa)^{\text {comp }}$ sharing with $(X, \omega)$ the separatrix diagram $\Gamma$ and the vector of heights $h$. The subset $\mathcal{H}(\kappa)_{\Gamma, h}^{\mathrm{comp}}$ coincides with the Re-leaf through $(X, \omega)$.

Proof. The collection of parameters $\ell_{i}$, where $i=1, \ldots, d-1$, and $t_{j}+i \cdot h_{j}$, where $j=1, \ldots, m$, associated with the separatrix diagram $\Gamma$ represents relative periods over a generating family of relative cycles; see Lemma 4.19. Thus, keeping the vector of heights $h$ fixed and deforming the parameters $\ell_{i}, t_{j}$, we 
stay inside the Re-leaf through $(X, \omega)$. On the other hand, Lemma 4.18 implies that the space of $\left(\ell_{i}, t_{j}\right)$-parameters has the same dimension $d=\operatorname{dim}_{\mathbb{R}} H^{1}(X$, $\Sigma ; \mathbb{R})$ as the Re-leaf. This implies that the connected component of $\mathcal{H}(\kappa)_{\Gamma, h}^{\text {comp }}$ containing $(X, \omega)$ coincides with the Re-leaf passing through $(X, \omega)$.

It remains to note that the set $\mathcal{H}(\kappa)_{\Gamma, h}^{\text {comp }}$ is connected. Indeed, in $(\ell, t, h)$ coordinates, the coordinate $h$ is fixed; the coordinates $t \in \mathbb{R}^{m}$ can be chosen arbitrarily and the coordinate vector $\ell$ can be chosen as an arbitrary vector in the intersection of $\mathbb{R}_{+}^{d-1}$ with the linear subspace defined by equations (4.17). The latter intersection is, clearly, connected.

Similarly, let $\Gamma$ be the separatrix diagram corresponding to a Jenkins-Strebel quadratic differential $(X, q)$. As before, denote by $h$ the vector of heights of the associated maximal horizontal cylinders. Let $\mathcal{Q}(\xi)^{\text {comp }}$ be the connected component of the ambient stratum containing $(X, q)$. Let $\mathcal{L}$ be the arithmetic invariant suborbifold obtained by the canonical double cover construction from $\mathcal{Q}(\xi)^{\text {comp }}$ (see Section 4.3 ); let $\mathcal{H}(\kappa)$ be the stratum of Abelian differentials ambient for $\mathcal{L}$. Let $(\hat{X}, \omega) \in \mathcal{L}$ be the Abelian differential representing the canonical double cover $p: \hat{X} \rightarrow X$ such that $p^{*} q=\omega^{2}$.

Consider the subset $\mathcal{Q}(\xi)_{\Gamma, h}^{\text {comp }} \subset \mathcal{Q}(\xi)^{\text {comp }}$ of all Jenkins-Strebel differentials in $\mathcal{Q}(\xi)^{\text {comp }}$ sharing with $(X, q)$ the separatrix diagram $\Gamma$ and the vector of heights $h$. Consider the subset obtained by applying the double cover construction to Jenkins-Strebel quadratic differentials in $\mathcal{Q}(\xi)_{\Gamma, h}^{\text {comp }}$. Let $\mathcal{L}_{\Gamma, h} \subset \mathcal{L}$ be the connected component of this set containing $(\hat{X}, \omega)$.

PROPOSITION 4.21. The subset $\mathcal{L}_{\Gamma, h}(\xi)$ coincides with the Re-leaf of $\mathcal{L}$ passing through $(\hat{X}, \omega)$.

Proof. The proof is analogous to the Proof of Proposition 4.20.

As it was already implicitly done above, we always assume that the maximal horizontal cylinders associated with a separatrix diagram are numbered from 1 to $m$ and that the horizontal saddle connections are numbered from 1 to $d-1$. Suppose that a Jenkins-Strebel differential $(X, \omega)$ has separatrix diagram $\Gamma$ and associated parameters $(\ell, h, t)$. A collection of parameters $\left(\ell^{\prime}, h^{\prime}, t^{\prime}\right)$ determine the same translation surface $(X, \omega)$ if and only if $\ell=\ell^{\prime}, h=h^{\prime}$ and

$$
t_{j}-t_{j}^{\prime} \equiv 0\left(\bmod w_{j}\right), \quad \text { for } j=1,2, \ldots, m,
$$

where $w_{j}=w_{\Gamma, j}^{\text {bot }}(\ell)=w_{\Gamma, j}^{\text {bot }}\left(\ell^{\prime}\right)$. For this reason, the parameters $t_{j}$ are only relevant modulo $w_{j}$. 


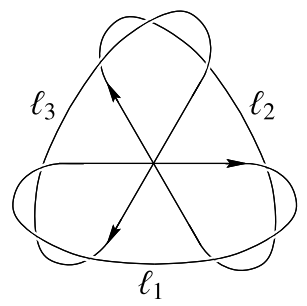

$\Gamma_{1}$

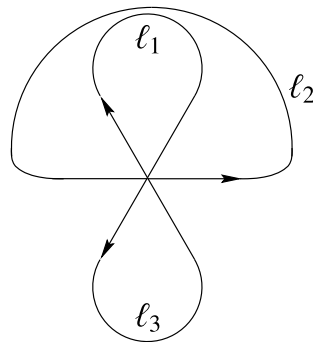

$\Gamma_{2}$

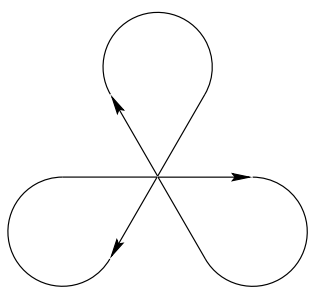

$\Gamma_{3}$

Figure 7. Ribbon graphs $\Gamma_{1}$ and $\Gamma_{2}$ are realizable as separatrix diagrams while $\Gamma_{3}$ is not.

A square-tiled surface always represents a Jenkins-Strebel differential. In the coordinate system as above, a Jenkins-Strebel differential corresponds to a square-tiled surface if and only if the metric data $(\ell, h, t)$ is integer.

Note that a general ribbon graph $\Gamma$ does not necessarily represent a separatrix diagram of some Jenkins-Strebel Abelian differential. The horizontal foliation of a Jenkins-Strebel Abelian differential is oriented, so the ribbon graph $\Gamma$ should be orientable: it should admit orientation of edges in such a way that at every vertex, the incoming edges and outgoing edges alternate with respect to the cyclic order induced by the ribbon structure. The pairing of boundary components should respect orientation of the surface. And finally, the system of equations (4.17) should admit a strictly positive solution $\left(\ell_{1}, \ldots, \ell_{d-1}\right)$.

EXAMPLE 4.22. Figure 7 represents all oriented ribbon graphs having a single vertex of valence 6 . Thus, any separatrix diagram realized by a Jenkins-Strebel differential from the stratum $\mathcal{H}(2)$ is represented by one of these three ribbon graphs.

The graph $\Gamma_{1}$ has two boundary components, so there is a unique way to glue a cylinder to this ribbon graph. We have already seen that in the case of a 1cylinder separatrix diagram, the system of equations (4.17) degenerates to an identity, which in the particular case of $\Gamma_{1}$ has the form

$$
\ell_{1}+\ell_{2}+\ell_{3}=\ell_{1}+\ell_{3}+\ell_{2} \text {. }
$$

Thus, $\Gamma_{1}$ is realizable as a separatrix diagram, which is the unique 1-cylinder separatrix diagram in $\mathcal{H}(2)$. Any collection of parameters $(\ell, t, h)$ with $\ell \in \mathbb{R}_{+}^{3}$, $t \in \mathbb{R}, h \in \mathbb{R}_{+}$defines a legal Jenkins-Strebel differential in $\mathcal{H}(2)$. Restricting the twist parameter to the subdomain $0 \leqslant t<\ell_{1}+\ell_{2}+\ell_{3}=w_{1}$, we get a polyhedral cone representing a single coordinate chart for all Jenkins-Strebel 
differentials in $\mathcal{H}(2)$ corresponding to this separatrix diagram. Any point of the polyhedral cone defines a unique well-defined Jenkins-Strebel differentials in $\mathcal{H}(2)$. Up to the symmetry of order 3 which cyclically changes the coordinates $\left(\ell_{1}, \ell_{2}, \ell_{3}\right)$, every 1 -cylinder Jenkins-Strebel differentials in $\mathcal{H}(2)$ is represented by a unique point $(\ell, t, h)$ of the resulting polyhedral cone.

The graph $\Gamma_{2}$ has four boundary components. However, there is a unique way to glue two cylinders to this ribbon graph in such a way that the resulting closed surface would be orientable and that the system of equations (4.17) admits a strictly positive solution. Namely, we have to glue one of the two cylinders to the inner boundary component of the loop $\ell_{1}$ and to the inner boundary component of the loop $\ell_{3}$, and the other cylinder to the remaining pair of boundary components. This time, the system of equations (4.17) imposes a nontrivial constraint $\ell_{1}=\ell_{3}$. As a coordinate chart in the space of parameters $(\ell, t, h)$, we can choose the following polyhedron. Take the intersection of the strictly positive octant $\mathbb{R}_{+}^{3}$ with the plane $\ell_{1}=\ell_{3}$ for the parameters $\ell$. Let $0 \leqslant t_{1}<\ell_{1}$ and $0 \leqslant t_{2}<\ell_{1}+\ell_{2}$. Let $h \in \mathbb{R}_{+}^{2}$. Any Jenkins-Strebel differential having this separatrix diagram can be represented by appropriate parameters $(\ell$, $t, h)$ in this polyhedral cone, and distinct points of the polyhedral cone represent distinct (and well-defined) Jenkins-Strebel differentials.

It is easy to verify that the ribbon graph $\Gamma_{3}$ is not realizable as a separatrix diagram: no matter how we arrange the boundary components into pairs, system (4.17) does not admit any strictly positive solution.

By Proposition 4.20 , every subset $\mathcal{H}(\kappa)_{\Gamma, h}^{\text {comp }}$ obtained by fixing the collection $h$ of heights of cylinders of a Jenkins-Strebel differential in $\mathcal{H}(\kappa)^{\text {comp }}$ and by varying the length parameters $(\ell, t)$ associated with the corresponding separatrix diagram $\Gamma$ coincides with a Re-leaf in $\mathcal{H}(\kappa)^{\text {comp }}$. Geometrically, each such $\mathcal{H}(\kappa)_{\Gamma, h}^{\text {comp }}$ is a torus bundle. The base of this bundle is the polyhedral cone $C_{+}(\Gamma)$ (or possibly its quotient with respect to a finite symmetry group) obtained as the intersection of $\mathbb{R}_{+}^{d-1}$ with the linear subspace (4.17). The fiber over a point $\ell$ in $C_{+}(\Gamma)$ is the 'twist torus'

$$
\mathbb{T}^{m}=\mathbb{R}^{m} /\left(w_{1} \mathbb{Z} \oplus w_{2} \mathbb{Z} \oplus \cdots \oplus w_{m} \mathbb{Z}\right),
$$

where $w_{1}(\ell), \ldots, w_{m}(\ell)$ are the perimeters of the cylinders.

Some of the separatrix diagrams realizable for the ambient stratum $\mathcal{H}(\kappa)$ might not be necessarily realizable in a given invariant arithmetic suborbifold $\mathcal{L}$. For example, any Jenkins-Strebel differential in the $\mathrm{GL}_{2}(\mathbb{R})$-orbit $\mathcal{L}$ of the Eierlegende Wollmilchsau represented in Figure 8 has the same separatrix diagram as the Eierlegende Wollmilchsau. In particular, $\mathcal{L}$ does not contain a single 1-cylinder square-tiled surface. 


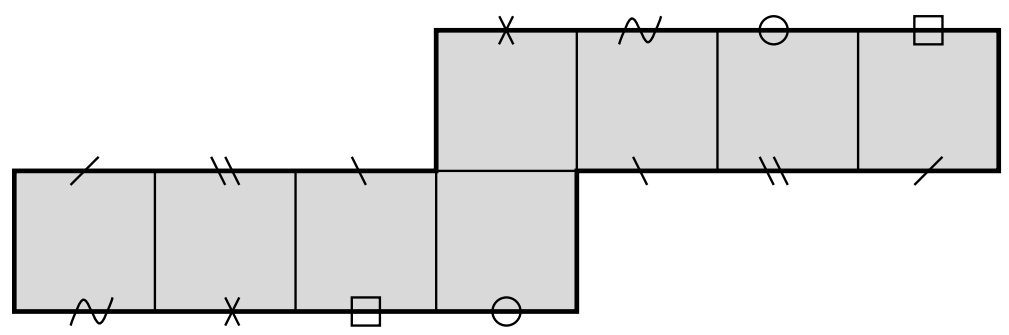

Figure 8. Eierlegende Wollmilchsau. As any square-tiled surface, it has a closed $\mathrm{GL}_{2}(\mathbb{R})$-orbit.

Given an invariant arithmetic suborbifold $\mathcal{L}$, we say that a separatrix diagram $\Gamma$ is realizable in $\mathcal{L}$ if $\mathcal{L}$ contains a Jenkins-Strebel differential having the separatrix diagram $\Gamma$. We call a vector $h=\left(h_{1}, h_{2}, \ldots, h_{m}\right)$ admissible for a separatrix diagram $\Gamma$ in $\mathcal{L}$ if there is a Jenkins-Strebel differential in $\mathcal{L}$ with separatrix diagram $\Gamma$ and with horizontal cylinders of heights $h$.

\subsection{Densities of subsets of square-tiled surfaces with prescribed}

horizontal or vertical decompositions. We now apply the results of Sections 4.5-4.7 to square-tiled surfaces with fixed combinatorics. In particular, we prove Corollary 4.25 that generalizes Theorem 2.6 from the Section 1.

Though we state the main results only for the connected components of the strata of Abelian and quadratic differentials, all the results of this section are valid for any invariant arithmetic suborbifold. However, we postpone these more general proofs to a separate paper [DGZZ2] to avoid overloading the current one.

In Section 4.3, we assigned to every connected component $\mathcal{Q}(\xi)^{\text {comp }}$ of every stratum of meromorphic quadratic differentials with at most simple poles an invariant arithmetic orbifold $\mathcal{L}$ in an appropriate stratum of Abelian differentials. By convention, everywhere in Section 4, we mark the preimages of simple poles on the cover. By Proposition 4.3 , the square-tiled surfaces in $\mathcal{Q}(\xi)^{\text {comp }}$ and in $\mathcal{L}$ are in the natural correspondence. In particular, we have direct relation between densities of the corresponding subsets of square-tiled surfaces measured in $\mathcal{Q}(\xi)^{\text {comp }}$ and in $\mathcal{L}$.

Consider a connected component $\mathcal{L}$ of some stratum of Abelian or quadratic differentials and a separatrix diagram $\Gamma$ realizable in it. Given an integer height vector $h$ admissible for $\Gamma$, we denote by $\mathcal{L}_{\mathbb{Z}, \Gamma, h}^{\text {horiz }}$ the set of square-tiled surfaces in $\mathcal{L}_{\mathbb{Z}}$ having separatrix diagram $\Gamma$ and vector $h$ of cylinder heights. We define $\mathcal{L}_{\mathbb{Z}, \Gamma, h}^{\text {vert }}$ as the set of square-tiled surfaces obtained from square-tiled surfaces in $\mathcal{L}_{\mathbb{Z}, \Gamma, h}^{\text {horiz }}$ by rotation by $\frac{\pi}{2}$ clockwise. 
THEOREM 4.23. Let $\mathcal{L}$ be a connected component of a stratum of Abelian differentials or an invariant arithmetic suborbifold in a stratum of Abelian differentials obtained by the canonical double cover construction from a connected component of a stratum of quadratic differentials. Let $\Gamma$ be a separatrix diagram realizable in $\mathcal{L}$. Then for any integer height vector $h$ admissible for $\Gamma$, the subset $\mathcal{L}_{\mathbb{Z}, \Gamma, h}^{\text {horiz }}$ of $\mathcal{L}_{\mathbb{Z}}$ has a nonzero density. Moreover, $c\left(\mathcal{L}_{\mathbb{Z}, \Gamma, h}^{\text {horiz }}\right)=\delta\left(\mathcal{L}_{\mathbb{Z}, \Gamma, h}^{\text {horiz }}\right) \cdot \operatorname{Vol}_{1}\left(\mathcal{L}_{1}\right)$ is a rational number, and we have the following explicit convergence rate as $N \rightarrow \infty$ :

$$
\mathcal{N}_{\mathcal{L}_{\mathbb{Z}, I, h}^{\text {horiz }}}(\mathcal{L}, N)=c\left(\mathcal{L}_{\mathbb{Z}, \Gamma, h}^{\text {horiz }}\right) \cdot \frac{N^{d}}{2 d}+O\left(N^{d-1}\right),
$$

where $d=\operatorname{dim}_{\mathbb{C}} \mathcal{L}$.

Proof. We start the proof with the case when $\mathcal{L}$ is a connected component of a stratum of Abelian differentials.

Let $(\ell, t, h)$ be parameters of the horizontal cylinder decomposition of a surface from $\mathcal{L}_{\mathbb{Z}, \Gamma, h}^{\text {horiz }}$. Then $\ell$ satisfies (4.17), so the width $w_{\Gamma, j}(\ell)$ of the $j$ th cylinder satisfies $w_{\Gamma, j}(\ell)=w_{\Gamma, j}^{\text {top }}(\ell)=w_{\Gamma, j}^{\text {bot }}(\ell)$. Hence, the flat area of the surface can be expressed in $(\ell, h)$-coordinates as

$$
\operatorname{Area}(\ell, h, t)=\sum_{j=1}^{m} h_{j} \cdot w_{\Gamma, j}(\ell) .
$$

Let $L_{\Gamma}$ be the linear subspace of $\mathbb{R}^{d-1} \times \mathbb{R}^{m}$ consisting of couples $(\ell, t)$ satisfying the system of linear equations (4.17).

Let $P_{\Gamma, h}$ be the polyhedron in $L_{\Gamma}$ defined by the following inequalities:

(1) $\ell$ is strictly positive,

(2) for each $j \in\{1, \ldots, m\}, 0 \leqslant t_{j}<w_{j}(\ell)$,

(3) $\operatorname{Area}(\ell, h, t) \leqslant 1$.

It follows from the definition of a separatrix diagram that $P_{\Gamma, h}$ is a relatively compact rational polyhedron of full dimension in $L_{\Gamma}$. This implies that $\mathrm{Vol} P_{\Gamma, h}$ is a (finite) strictly positive rational number, where $\mathrm{Vol}$ is the Lebesgue measure in the vector space $L_{\Gamma}$ normalized in such a way that the fundamental domain of the lattice $L_{\Gamma} \cap\left(\mathbb{Z}^{d-1} \times \mathbb{Z}^{m}\right)$ has volume 1 .

Up to a possible symmetry of finite order, square-tiled surfaces tiled with at most $N$ unit squares in $\mathcal{L}_{\mathbb{Z}, \Gamma, h}^{\text {horiz }}$ are in the one-to-one correspondence with the integral points in the inflated polyhedron $N \cdot P_{\Gamma, h}$ (where the inflation does not 
affect the parameters $h$ and acts on the space $\mathbb{R}^{d-1} \times \mathbb{R}^{m}$ of $(\ell, t)$-coordinates by homothety with coefficient $N$ ). Hence,

$$
\begin{aligned}
\mathcal{N}\left(\mathcal{L}_{\mathbb{Z}, \Gamma, h}^{\text {horiz }}, N\right) & =\frac{1}{\operatorname{Aut}(\Gamma, h)} \cdot \operatorname{Card}\left(N \cdot P_{\Gamma, h} \cap\left(\mathbb{Z}^{d-1} \times \mathbb{Z}^{m}\right)\right) \\
& =\frac{1}{\operatorname{Aut}(\Gamma, h)} \cdot \operatorname{Vol}\left(P_{\Gamma, h}\right) \cdot N^{\operatorname{dim}\left(P_{\Gamma}\right)}+O\left(N^{\operatorname{dim}\left(P_{\Gamma}\right)-1}\right),
\end{aligned}
$$

where $\operatorname{dim}\left(P_{\Gamma}\right)=\operatorname{dim}\left(L_{\Gamma}\right)=\operatorname{dim}_{\mathbb{C}} \mathcal{L}$ by Proposition 4.20. By equation (4.11), this implies that $\mathcal{L}_{\mathbb{Z}, \Gamma, h}^{\text {horiz }}$ has strictly positive density $\delta\left(\mathcal{L}_{\mathbb{Z}, \Gamma, h}^{\text {horiz }}\right)$ and that the quantity

$$
c\left(\mathcal{L}_{\mathbb{Z}, \Gamma, h}^{\text {horiz }}\right)=\delta\left(\mathcal{L}_{\mathbb{Z}, \Gamma, h}^{\text {horiz }}\right) \cdot \operatorname{Vol}_{1}\left(\mathcal{L}_{1}\right)=\operatorname{dim}_{\mathbb{R}} \mathcal{L} \cdot \frac{\operatorname{Vol}\left(P_{\Gamma, h}\right)}{\operatorname{Aut}(\Gamma, h)}
$$

is a rational number.

Suppose now that $\mathcal{L}$ is an invariant arithmetic suborbifold in an appropriate stratum $\mathcal{H}(\kappa)$ of Abelian differentials obtained by the canonical double cover construction from a connected component $\mathcal{Q}(\xi)^{\text {comp }}$ of a stratum of meromorphic quadratic differentials with at most simple poles. Any realizable separatrix diagram $\hat{\Gamma}$ in $\mathcal{L}$ is induced from a realizable separatrix diagram $\Gamma$ in the original connected component of a stratum of quadratic differentials. By convention, we mark all preimages of simple poles (if any).

It is convenient to denote by $(\ell, t, h)$ the lengths of horizontal saddle connections, the twist parameters and the heights of the cylinders of the original Jenkins-Strebel quadratic differential $(X, q) \in \mathcal{Q}(\xi)^{\text {comp }}$. Suppose that the original separatrix diagram $\Gamma$ contains $m$ cylinders, that $\gamma_{1}, \ldots, \gamma_{m}$ represent the nonhorizontal saddle connections crossing these cylinders and that $\lambda_{1}, \ldots$, $\lambda_{d}$ denote the horizontal saddle connections. By Lemma 4.17, the number $d$ of horizontal saddle connections coincides with the dimension $d=\operatorname{dim}_{\mathbb{C}} \mathcal{Q}(\xi)$ of the ambient stratum of quadratic differentials. By construction, the separatrix diagram $\hat{\Gamma}$ corresponding to the Jenkins-Strebel differential on the double cover has $2 m$ cylinders.

Every horizontal saddle connection $\lambda_{i}$ on $(X, q)$ has two preimages $\lambda_{i}^{\prime}$ and $\lambda_{i}^{\prime \prime}$ on $(\hat{X}, \omega)$. Every nonhorizontal saddle connection $\gamma_{j}$ also has two preimages $\gamma_{j}^{\prime}$ and $\gamma_{j}^{\prime \prime}$ crossing the two maximal horizontal cylinders on the cover corresponding to two copies of the horizontal cylinder number $j$ on the base. The induced collection of parameters $\left(\ell^{\prime}, \ell^{\prime \prime}, t^{\prime}, t^{\prime \prime}, h^{\prime}, h^{\prime \prime}\right)$ serves as the complete collection of length parameters on the covering Jenkins-Strebel Abelian differential with the separatrix diagram $\hat{\Gamma}$. By construction, they satisfy 
the obvious relations

$$
\begin{cases}\ell_{i}^{\prime}=\ell_{i}^{\prime \prime} & \text { for } i=1, \ldots, d, \\ t_{j}^{\prime}=t_{j}^{\prime \prime} & \text { for } j=1, \ldots, m, \\ h_{j}^{\prime}=h_{j}^{\prime \prime} & \text { for } j=1, \ldots, m,\end{cases}
$$

where, by construction, we have $\ell_{j}^{\prime}=\ell_{j}^{\prime \prime}=\ell_{j}$ for $j=1, \ldots, d$ as well as $t_{j}^{\prime}=t_{j}^{\prime \prime}=t_{j}$ for $j=1, \ldots, m$ and $h_{j}^{\prime}=h_{j}^{\prime \prime}=h_{j}$ for $j=1, \ldots, m$.

Consider the linear subspace $L_{\Gamma} \subset \mathbb{R}^{m-1} \times \mathbb{R}^{m}$ of simultaneous solutions of the following two systems of linear equations: system (4.17) associated with the separatrix diagram $\hat{\Gamma}$ and system (4.19). Suppose that some collection $\left(\ell^{\prime}\right.$, $\left.\ell^{\prime \prime}, t^{\prime}, t^{\prime \prime}, h^{\prime}, h^{\prime \prime}\right)$ with strictly positive $\ell^{\prime}, \ell^{\prime \prime}, h^{\prime}, h^{\prime \prime}$ defines a point of $L_{\Gamma}$. Define $\ell_{j}:=\ell_{j}^{\prime}=\ell_{j}^{\prime \prime}$ for $j=1, \ldots, d$; define $t_{j}:=t_{j}^{\prime}=t_{j}^{\prime \prime}=t_{j}$ for $j=1, \ldots, m$ and define $h_{j}:=h_{j}^{\prime}=h_{j}^{\prime \prime}$ for $j=1, \ldots, m$. The collection $(\ell, t, h)$ defines a Jenkins-Strebel differential in $\mathcal{Q}(\xi)^{\text {comp }}$ with separatrix diagram $\Gamma$ and vector of heights $h$. Reciprocally, any Jenkins-Strebel differential in $\mathcal{Q}(\xi)^{\text {comp }}$ with separatrix diagram $\Gamma$ and vector of heights $h$ defines a point in $L_{\Gamma}$.

The rest of the proof is completely analogous to the case of Abelian differentials treated above.

Applying the results from Sections 4.5 and 4.6, we obtain the following corollary.

COROLlaRY 4.24. Let $\mathcal{L}$ be a connected component of a stratum of Abelian differentials or an invariant arithmetic suborbifold in a stratum of Abelian differentials obtained by the canonical double cover construction from a connected component of a stratum of quadratic differentials. Let $\Gamma$ and $\Gamma^{\prime}$ be realizable separatrix diagrams in $\mathcal{L}$ and let $h$ and $h^{\prime}$ be admissible integer vectors of heights of cylinders for $\Gamma$ and $\Gamma^{\prime}$, respectively. Then all of $\mathcal{L}_{\mathbb{Z}, \Gamma, h}^{\text {horiz }}$, $\mathcal{L}_{\mathbb{Z}, \Gamma^{\prime}, h^{\prime}}^{\text {vert }}$ and $\mathcal{L}_{\mathbb{Z}, \Gamma, h}^{\text {horiz }} \cap \mathcal{L}_{\mathbb{Z}, \Gamma^{\prime}, h^{\prime}}^{\text {vert }}$ have strictly positive uniform densities in $\mathcal{L}$ and

$$
\delta\left(\mathcal{L}_{\mathbb{Z}, \Gamma, h}^{\text {horiz }} \cap \mathcal{L}_{\mathbb{Z}, \Gamma^{\prime}, h^{\prime}}^{\text {vert }}\right)=\delta\left(\mathcal{L}_{\mathbb{Z}, \Gamma, h}^{\text {horiz }}\right) \cdot \delta\left(\mathcal{L}_{\mathbb{Z}, \Gamma^{\prime}, h^{\prime}}^{\text {vert }}\right),
$$

where $\delta\left(\mathcal{L}_{\mathbb{Z}, \Gamma^{\prime}, h^{\prime}}^{\text {vert }}\right)=\delta\left(\mathcal{L}_{\mathbb{Z}, \Gamma^{\prime}, h^{\prime}}^{\text {horiz }}\right)$.

Proof. Theorem 4.23 shows that the set $\mathcal{L}_{\mathbb{Z}, \Gamma, h}^{\text {horiz }}$ has a strictly positive density. By Proposition 4.20 (respectively by Proposition 4.21 in the case of quadratic differentials), the set $\mathcal{L}_{\mathbb{Z}, \Gamma, h}^{\text {horiz }}$ is Re-invariant, which allows us to use Theorem 4.16 and conclude that the density is, actually, uniform.

The analogous results hold for the vertically completely periodic surfaces with separatrix diagram $\Gamma^{\prime}$, namely, by the same arguments, the set $\mathcal{L}_{\mathbb{Z}, \Gamma^{\prime}, h^{\prime}}^{\text {vert }}$ has uniform strictly positive density and is Im-invariant. 
Theorem 4.16 implies that the intersection $\mathcal{L}_{\Gamma, h}^{\text {horiz }} \cap \mathcal{L}_{\Gamma^{\prime}, h^{\prime}}^{\text {vert }}$ has uniform strictly positive density equal to the product of the densities of the two sets.

To complete the proof, note that the set $\mathcal{L}_{\mathbb{Z}, \Gamma^{\prime}, h^{\prime}}^{\text {vert }}$ is constructed by applying to each square-tiled surface in the set $\mathcal{L}_{\mathbb{Z}, \Gamma^{\prime}, h^{\prime}}^{\text {hori }}$ clockwise rotation by $\frac{\pi}{2}$. Thus, $\mathcal{N}_{\mathcal{L}_{\mathbb{Z}, \Gamma^{\prime}, h^{\prime}}^{\text {vert }}}(\mathcal{L}, N)=\mathcal{N}_{\mathcal{L}_{\mathbb{Z}, \Gamma^{\prime}, h^{\prime}}^{\text {horiz }}}(\mathcal{L}, N)$. The equality $\delta\left(\mathcal{L}_{\mathbb{Z}, \Gamma^{\prime}, h^{\prime}}^{\text {vert }}\right)=\delta\left(\mathcal{L}_{\mathbb{Z}, \Gamma^{\prime}, h^{\prime}}^{\text {horiz }}\right)$ now follows directly from Definition (4.10) of the density.

In Section 2.1, we assigned to every square-tiled surfaces on the sphere a pair of transverse multicurves. Analogously, one can assign to any square-tiled surface in a stratum of Abelian differential $\mathcal{H}(\kappa)$ a pair of transverse multicurves composed of all closed regular flat geodesics passing through the centers of the squares.

Recall that closed regular horizontal geodesics on a square-tiled surface $(X, \omega$, $\Sigma$ ) are organized into maximal horizontal cylinders having at least one point of the set $\Sigma$ on each of the boundary components and no points of $\Sigma$ in the interior of the cylinder. All regular closed horizontal geodesics inside each maximal horizontal cylinder belong to the same free homotopy class on $X \backslash \Sigma$. A similar property is valid for the vertical cylinder decomposition. This observation allows us to express the horizontal (respectively vertical) multicurve associated with a square-tiled surface in terms of the horizontal (respectively vertical) cylinder decomposition as the weighted sum $h_{1} \gamma_{1}+h_{2} \gamma_{2}+\cdots+h_{m} \gamma_{m}$, where $h_{i}$ and $\gamma_{i}$ are, respectively, the height and the core curve of the $i$ th cylinder in this decomposition. Here by the 'height' of a square-tiled cylinder, we mean the number of circular bands of squares which form the cylinder. In particular, the number of components of the resulting horizontal (respectively vertical) multicurve counted with weights is the total height $h_{1}+h_{2}+\cdots+h_{m}$ of all maximal horizontal (respectively vertical) cylinders.

Let $\mathcal{L}$ be a connected component of a stratum of Abelian differentials or the invariant arithmetic orbifold obtained by the canonical double cover construction from a component of a stratum of quadratic differentials. Denote by $\mathcal{L}_{\mathbb{Z}, k}^{\text {horiz }}$ (respectively by $\mathcal{L}_{\mathbb{Z}, k}^{\text {vert }}$ ) the subset of square-tiled surfaces in $\mathcal{L}$ whose associated horizontal (respectively vertical) multicurve has $k$ components. In other words, $\mathcal{L}_{\mathbb{Z}, k}^{\text {horiz }}$ (respectively $\mathcal{L}_{\mathbb{Z}, k}^{\text {vert }}$ ) is the subset of square-tiled surfaces in $\mathcal{L}$ tiled with unit squares arranged into exactly $k$ horizontal (respectively vertical) bands of squares. In terms of the decomposition into maximal horizontal (respectively vertical cylinders), we have $k=h_{1}+\cdots+h_{m}$, where $h_{1}, \ldots, h_{m}$ are the heights of the cylinders.

COROLlary 4.25. Let $\mathcal{L}$ be a connected component of a stratum of Abelian differentials or the invariant arithmetic orbifold obtained by the canonical 
double cover construction from a component of a stratum of quadratic differentials. Let $k, k_{h}, k_{v} \in \mathbb{N}$ be positive integers in the case of Abelian differentials and even positive integers in the case of meromorphic quadratic differentials with at most simple poles.

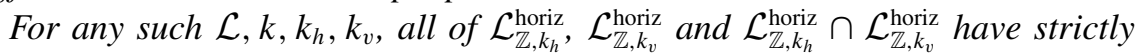
positive uniform densities in $\mathcal{L}$ and

$$
\delta\left(\mathcal{L}_{\mathbb{Z}, k_{h}}^{\text {horiz }} \cap \mathcal{L}_{\mathbb{Z}, k_{v}}^{\text {vert }}\right)=\delta\left(\mathcal{L}_{\mathbb{Z}, k_{h}}^{\text {horiz }}\right) \cdot \delta\left(\mathcal{L}_{\mathbb{Z}, k_{v}}^{\text {vert }}\right),
$$

where $\delta\left(\mathcal{L}_{\mathbb{Z}, k}^{\text {horiz }}\right)=\delta\left(\mathcal{L}_{\mathbb{Z}, k}^{\text {vert }}\right)$.

Moreover, $c\left(\mathcal{L}_{\mathbb{Z}, k}\right)=\delta\left(\mathcal{L}_{\mathbb{Z}, k}^{\text {horiz }}\right) \cdot \operatorname{Vol}_{1}\left(\mathcal{L}_{1}\right)$ is a rational number and we have the explicit convergence rate

$$
\mathcal{N}_{\mathcal{L}_{\mathbb{Z}, k}^{\text {horiz }}}(\mathcal{L}, N)=\mathcal{N}_{\mathcal{L}_{\mathbb{Z}, k}^{\text {vert }}}(\mathcal{L}, N)=c\left(\mathcal{L}_{\mathbb{Z}, k}\right) \cdot \frac{N^{d}}{2 d}+O\left(N^{d-1}\right),
$$

where $d=\operatorname{dim}_{\mathbb{C}} \mathcal{L}$.

Proof. The proof follows from Corollary 4.24. Note that each stratum has only a finite number of separatrix diagrams and hence

$$
\mathcal{L}_{\mathbb{Z}, k}^{\text {horiz }}=\bigsqcup_{\Gamma} \bigsqcup_{h=\left(h_{1}, \ldots, h_{m}\right)} \mathcal{L}_{\mathbb{Z}, \Gamma, h}^{\text {horiz }}
$$

Here the first union is taken with respect to the finite set of separatrix diagrams realizable in $\mathcal{L}$. For each such separatrix diagram, we denote by $m=m(\Gamma)$ the number of maximal horizontal cylinders associated with $\Gamma$. The second (finite) union is taken with respect to all positive integer heights $h_{1}, \ldots, h_{m}$ admissible for $\Gamma$ in $\mathcal{L}$ and satisfying the relation $h_{1}+\cdots+h_{m}=k$. This proves that for any integer $k$, the set $\mathcal{L}_{\mathbb{Z}, k}^{\text {horiz }}$ has uniform density satisfying the relation

$$
\delta\left(\mathcal{L}_{\mathbb{Z}, k}^{\text {horiz }}\right)=\sum_{\Gamma} \sum_{h} \delta\left(\mathcal{L}_{\mathbb{Z}, \Gamma, h}^{\text {horiz }}\right)
$$

where the $\delta\left(\mathcal{L}_{\mathbb{Z}, \Gamma, h}^{\text {horiz }}\right)$ are the densities appearing in Corollary 4.24.

To justify that for any $k \in \mathbb{N}$, in the case when $\mathcal{L}$ is a component of a stratum of Abelian differentials (respectively, for any $k \in 2 \mathbb{N}$ in the case when $\mathcal{L}$ is an invariant arithmetic orbifold associated with a component of a stratum of quadratic differentials) $\delta\left(\mathcal{L}_{\mathbb{Z}, k}^{\text {horiz }}\right)$ is a strictly positive number, it is sufficient to use the fact that each component of any stratum of Abelian or quadratic differentials admits a 1-cylinder square-tiled surface; see [KoZo] and [Zor3]. Considering 
the associated separatrix diagram $\Gamma$, we conclude that the term $\delta_{\mathbb{Z}, \Gamma, k}$ is already strictly positive.

The proof that $\mathcal{L}_{\mathbb{Z}, k}^{\text {vert }}$ has strictly positive uniform density is completely analogous. The proof that $\mathcal{L}_{\mathbb{Z}, k_{h}}^{\text {horiz }} \cap \mathcal{L}_{\mathbb{Z}, k_{v}}^{\text {horiz }}$ has uniform density equal to product of densities now follows from Theorem 4.16 in the same way as was done in Corollary 4.24.

Finally, the rationality of $c\left(\mathcal{L}_{\mathbb{Z}, k}\right)$ and the explicit convergence rate in the remaining assertion follow now from Theorem 4.23 applied to each of the terms in (4.21) and (4.22), respectively.

REMARK 4.26. We have seen that each of the sets $\mathcal{L}_{\mathbb{Z}, \Gamma, h}^{\text {horiz }}$ and $\mathcal{L}_{\mathbb{Z}, k_{h}}^{\text {horiz constructed }}$ above is Re-invariant. Similarly, the sets $\mathcal{L}_{\mathbb{Z}, \Gamma, h}^{\text {vert }}, \mathcal{L}_{\mathbb{Z}, k_{v}}^{\text {vert }}$ are Im-invariant. Thus, by Theorem 4.16, all these sets as well as any intersection of a set from the first group and a set from the second group has uniform density, and the density of the intersection is the product of densities of the two corresponding sets. We do not need to fix combinatorics of the horizontal and vertical cylinder decomposition in the same way: the statement is valid for any combination like $\mathcal{L}_{\mathbb{Z}, \Gamma, h}^{\text {horiz }} \cap \mathcal{L}_{\mathbb{Z}, k}^{\text {vert }}$.

We are ready to prove Theorems 2.5 and 2.6 with exception for the explicit value (2.7) for $\operatorname{cyl}_{1}\left(\mathcal{Q}\left(v,-1^{|v|+4}\right)\right)$ which will be proved in Section 4.10.

Proof of Theorems 2.5 and 2.6. By assertions (1) and (2) of Proposition 4.3, the number $\mathcal{S}_{k, v}^{\text {labeled }}(N)$ of square-tiled surfaces in the stratum $\mathcal{Q}\left(v,-1^{|v|+4}\right)$ with labeled zeros and poles tiled with at most $2 N$ squares organized into $k$ horizontal bands and the number $\mathcal{S}_{k_{h}, k_{v}, v}^{\text {labele }}(N)$ of square-tiled surfaces in the same stratum with labeled zeros and poles tiled with at most $2 N$ squares composed of $k_{h}$ horizontal and $k_{v}$ vertical bands of squares satisfies the following relations:

$$
\begin{aligned}
& \mathcal{S}_{k, v}^{\text {labeled }}(N)=\frac{1}{\operatorname{deg}(P)} \cdot \mathcal{N}_{\mathcal{L}_{\mathbb{Z}, k}^{\text {horiz }}}(\mathcal{L}, 4 N), \\
& \mathcal{S}_{k_{h}, k_{v}, v}^{\text {labeled }}(N)=\frac{1}{\operatorname{deg}(P)} \cdot \mathcal{N}_{\mathcal{L}_{\mathbb{Z}, k_{h}}^{\text {horiz }} \cap \mathcal{L}_{\mathbb{Z}, k_{v}}^{\text {vert }}}(\mathcal{L}, 4 N) .
\end{aligned}
$$

By Corollary 4.25, we have as $N \rightarrow+\infty$

$$
\begin{aligned}
\mathcal{N}_{\mathcal{L}_{\mathbb{Z}, k}^{\text {horiz }}}(\mathcal{L}, 4 N) & =\delta\left(\mathcal{L}_{\mathbb{Z}, k}^{\text {horiz }}\right) \cdot \operatorname{Vol}_{1}\left(\mathcal{L}_{1}\right) \cdot \frac{(4 N)^{d}}{2 d}+O\left(N^{d-1}\right) \\
& =\delta\left(\mathcal{L}_{\mathbb{Z}, k}^{\text {horiz }}\right) \cdot \operatorname{Vol}_{1}\left(\mathcal{L}_{1}\right) \cdot \frac{(4 N)^{d}}{2 d}+o\left(N^{d}\right) .
\end{aligned}
$$


Combining (4.23) with the latter expression and applying (4.7), we obtain

$$
\begin{aligned}
\mathcal{S}_{k, v}^{\text {labeled }}(N) & =\frac{1}{\operatorname{deg}(P)} \cdot \mathcal{N}_{\mathcal{L}_{\mathbb{Z}, k}^{\text {horiz }}}(\mathcal{L}, 4 N) \\
& =\delta\left(\mathcal{L}_{\mathbb{Z}, k}^{\text {horiz }}\right) \cdot \frac{4^{d}}{\operatorname{deg}(P)} \cdot \operatorname{Vol}_{1}\left(\mathcal{L}_{1}\right) \cdot \frac{N^{d}}{2 d}+O\left(N^{d-1}\right) \\
& =\delta\left(\mathcal{L}_{\mathbb{Z}, k}^{\text {horiz }}\right) \cdot \operatorname{Vol}_{1}\left(\mathcal{Q}_{1}\left(\nu,-1^{|\nu|+4}\right)\right) \cdot \frac{N^{d}}{2 d}+O\left(N^{d-1}\right) \quad \text { as } N \rightarrow+\infty .
\end{aligned}
$$

Letting

$$
\operatorname{cyl}_{k}\left(\mathcal{Q}\left(v,-1^{|v|+4}\right)\right):=\delta\left(\mathcal{L}_{\mathbb{Z}, k}^{\text {horiz }}\right) \cdot \operatorname{Vol}_{1}\left(\mathcal{Q}\left(v,-1^{|v|+4}\right)\right),
$$

we obtain the desired formula (2.6).

The rationality of $\operatorname{cyl}_{k}\left(\mathcal{Q}\left(v,-1^{|v|+4}\right)\right)$ follows from the rationality of $c\left(\mathcal{L}_{\mathbb{Z}, k}\right)$ proved in Corollary 4.25 combined with the relation

$$
\operatorname{cyl}_{k}\left(\mathcal{Q}\left(v,-1^{|v|+4}\right)\right)=\frac{\operatorname{deg}(P)}{4^{d}} \cdot c\left(\mathcal{L}_{\mathbb{Z}, k}\right) .
$$

This completes the Proof of Theorem 2.5 with exception for the explicit value of $\operatorname{cyl}_{1}\left(\mathcal{Q}\left(v,-1^{|v|+4}\right)\right)$ given by (2.7) which we compute in Section 4.10.

The Proof of Theorem 2.6 is analogous. Namely, by property (4.11) of a density of a subset $\mathcal{L}_{\mathbb{Z}, k_{h}}^{\text {horiz }} \cap \mathcal{L}_{\mathbb{Z}, k_{v}}^{\text {vert }}$ of $\mathcal{L}_{\mathbb{Z}}$, we have

$\mathcal{N}_{\mathcal{L}_{\mathbb{Z}, k_{h}}^{\text {horiz }} \cap \mathcal{L}_{\mathbb{Z}, k_{v}}^{\text {vert }}}(\mathcal{L}, 4 N)=\delta\left(\mathcal{L}_{\mathbb{Z}, k_{h}}^{\text {horiz }} \cap \mathcal{L}_{\mathbb{Z}, k_{v}}^{\text {vert }}\right) \cdot \operatorname{Vol}_{1}\left(\mathcal{L}_{1}\right) \cdot \frac{(4 N)^{d}}{2 d}+o\left(N^{d}\right)$ as $N \rightarrow+\infty$.

Combining (4.24) with the latter expression and applying (4.7) as above, we obtain

$$
\begin{aligned}
\mathcal{S}_{k_{h}, k_{v}, v}^{\text {labeled }}(N)= & \frac{1}{\operatorname{deg}(P)} \cdot \mathcal{N}_{\mathcal{L}_{\mathbb{Z}, k_{h}}^{\text {horiz }} \cap \mathcal{L}_{\mathbb{Z}, k_{v}}^{\text {vert }}}(\mathcal{L}, 4 N), \\
= & \delta\left(\mathcal{L}_{\mathbb{Z}, k_{h}}^{\text {horiz }} \cap \mathcal{L}_{\mathbb{Z}, k_{v}}^{\text {vert }}\right) \cdot \frac{4^{d}}{\operatorname{deg}(P)} \cdot \operatorname{Vol}_{1}\left(\mathcal{L}_{1}\right) \cdot \frac{N^{d}}{2 d}+o\left(N^{d}\right) \\
= & \delta\left(\mathcal{L}_{\mathbb{Z}, k_{h}}^{\text {horiz }} \cap \mathcal{L}_{\mathbb{Z}, k_{v}}^{\text {vert }}\right) \cdot \operatorname{Vol}_{1}\left(\mathcal{Q}_{1}\left(\nu,-1^{|v|+4}\right)\right) \cdot \frac{N^{d}}{2 d}+o\left(N^{d}\right) \\
& \quad \text { as } N \rightarrow+\infty .
\end{aligned}
$$

Letting

$$
\operatorname{cyl}_{k_{h}, k_{v}}\left(\mathcal{Q}\left(v,-1^{|v|+4}\right)\right):=\delta\left(\mathcal{L}_{\mathbb{Z}, k_{h}}^{\text {horiz }} \cap \mathcal{L}_{\mathbb{Z}, k_{v}}^{\text {vert }}\right) \cdot \operatorname{Vol}_{1}\left(\mathcal{Q}\left(v,-1^{|v|+4}\right)\right),
$$

we obtain formula (2.8). The remaining equation (2.9) is obtained by rewriting (4.20) in terms of the quantities $\operatorname{cyl}_{k}\left(\mathcal{Q}\left(v,-1^{|v|+4}\right)\right)$ and $\operatorname{cyl}_{k_{h}, k_{v}}(\mathcal{Q}(v$, $\left.-1^{|v|+4}\right)$ ) defined by expressions (4.25) and (4.26), respectively. Theorem 2.6 is proved. 
We complete this section with the following lemma used in the Proof of Theorem 3.7. We state it for strata of meromorphic quadratic differentials in genus zero, which allows us to use notations introduced in Section 3. However, both the statement and the proof of the lemma are applicable to any connected component of any stratum of Abelian or quadratic differentials up to adjustment of notations specific strata in genus zero.

Let $\Gamma$ be a separatrix diagram realizable in a stratum $\mathcal{Q}\left(v,-1^{|v|+4}\right)$, let $h$ be an admissible integer vector of heights and let $k$ be a positive integer. We denote by $\mathcal{S}_{v}^{\text {labeled }}(N)$ the number of all square-tiled surfaces in $\mathcal{Q}\left(v,-1^{|v|+4}\right)$ tiled with at most $2 N$ identical squares. We denote by $\mathcal{S}_{\Gamma, h, v}^{\text {labeled }}(N)$ the number of squaretiled surfaces as above with additional restriction that their horizontal cylinder decomposition is represented by $(\Gamma, h)$. We denote by $\mathcal{S}_{k, v}^{\text {labeled }}(N)$ the number of square-tiled surfaces in $\mathcal{Q}\left(v,-1^{|v|+4}\right)$ tiled with at most $2 N$ identical squares and having $k$ vertical bands of squares. Finally, we denote by $\mathcal{S}_{\Gamma, h, k, v}(N)$ the number of square-tiled surfaces as above with horizontal cylinder decomposition represented by $(\Gamma, h)$ and having $k$ vertical bands of squares.

LEMMA 4.27. For any stratum $\mathcal{Q}\left(v,-1^{|v|+4}\right)$, any separatrix diagram $\Gamma$ realizable in this stratum, any admissible integer vector $h$ of heights and any positive integer $k$, we have

$$
\lim _{N \rightarrow+\infty} \frac{\mathcal{S}_{\Gamma, h, k, v}^{\text {labeled }}(N)}{\mathcal{S}_{\Gamma, h, v}^{\text {labeled }}(N)}=\lim _{N \rightarrow+\infty} \frac{\mathcal{S}_{k, v}^{\text {labeled }}(N)}{\mathcal{S}_{v}^{\text {labeled }}(N)} .
$$

Proof. We follow the Proof of Theorems 2.5 and 2.6. Let $\mathcal{L}$ be the affine arithmetic orbifold associated with the stratum $\mathcal{Q}\left(v,-1^{|v|+4}\right)$. We have

$$
\begin{aligned}
& \mathcal{S}_{\Gamma, h, v}^{\text {labeled }}(N)=\frac{1}{\operatorname{deg}(P)} \cdot \mathcal{N}_{\mathcal{L}_{\mathbb{Z}, \Gamma, h}^{\text {horiz }}}(\mathcal{L}, 4 N), \\
& \mathcal{S}_{\Gamma, h, k, v}^{\text {labeled }}(N)=\frac{1}{\operatorname{deg}(P)} \cdot \mathcal{N}_{\mathcal{L}_{\mathbb{Z}, I, h}^{\text {horiz }} \cap \mathcal{L}_{\mathbb{Z}, k}^{\text {vert }}(\mathcal{L}, 4 N),}
\end{aligned}
$$

where the sets $\mathcal{L}_{\mathbb{Z}, \Gamma, h}^{\text {horiz }}$ and $\mathcal{L}_{\mathbb{Z}, k}^{\text {vert }}$ are as in Corollaries 4.24 and 4.25, respectively. Thus,

$$
\frac{\mathcal{S}_{\Gamma, h, k, v}^{\text {labeled }}(N)}{\mathcal{S}_{\Gamma, h, v}^{\text {labeled }}(N)}=\frac{\mathcal{N}_{\mathcal{L}_{\mathbb{Z}, \Gamma, h}^{\text {horiz }} \cap \mathcal{L}_{\mathbb{Z}, k}^{\text {vert }}}(\mathcal{L}, 4 N)}{\mathcal{N}_{\mathcal{L}_{\mathbb{Z}, \Gamma, h}^{\text {horiz }}}(\mathcal{L}, 4 N)} .
$$

By Remark 4.26, we have

$$
\delta\left(\mathcal{L}_{\mathbb{Z}, \Gamma, h}^{\text {horiz }} \cap \mathcal{L}_{\mathbb{Z}, k}^{\text {vert }}\right)=\delta\left(\mathcal{L}_{\mathbb{Z}, \Gamma, h}^{\text {horiz }}\right) \cdot \delta\left(\mathcal{L}_{\mathbb{Z}, k}^{\text {vert }}\right),
$$


which implies that

$$
\lim _{N \rightarrow+\infty} \frac{\mathcal{N}_{\mathcal{L}_{\mathbb{Z}, T, h}^{\text {horiz }} \cap \mathcal{L}_{\mathbb{Z}, k}^{\text {vert }}}(\mathcal{L}, 4 N)}{\mathcal{N}_{\mathcal{L}_{\mathbb{Z}, \Gamma, h}^{\text {horiz }}}(\mathcal{L}, 4 N)}=\lim _{N \rightarrow+\infty} \frac{\mathcal{N}_{\mathcal{L}_{\mathbb{Z}, k}^{\text {vert }}}(\mathcal{L}, 4 N)}{\mathcal{N}_{\mathcal{L}_{\mathbb{Z}}}(\mathcal{L}, 4 N)}
$$

Applying (4.23) and the analogous relation $\mathcal{S}_{v}^{\text {labeled }}(N)=\frac{1}{\operatorname{deg}(P)} \cdot \mathcal{N}_{\mathcal{L}_{\mathbb{Z}}^{\text {horiz }}}(\mathcal{L}, 4 N)$ to the expressions in the right-hand side of the latter equality, we complete the proof of the lemma.

4.9. Explicit densities for 1-cylinder diagrams on the sphere. In this section, we consider separatrix diagrams $\Gamma$ with a single cylinder in a stratum of quadratic differentials $\mathcal{Q}\left(v,-1^{|v|+4}\right)$ on the sphere. In the lemma below, we reproduce formula (2.2) from [DGZZ, Proposition 2.3] adapted to the language of the current paper.

Consider a Jenkins-Strebel meromorphic quadratic differential with simple poles on $\mathbb{C P}^{1}$. Suppose that it has a single maximal horizontal cylinder. The union of all horizontal saddle connections of such a Jenkins-Strebel differential forms two connected components and each of the two components is a tree. In other words, a 1-cylinder separatrix diagram $\Gamma$ on the sphere can be encoded by a pair of plane trees (one for each boundary component of the maximal horizontal cylinder). There is no ambiguity in such a definition since the corresponding ribbon graph has only two boundary components, so there is a single way to join the boundary components by the cylinder.

Let $\mathcal{Q}\left(v,-1^{|v|+4}\right)$ be a stratum of meromorphic quadratic differentials with at most simple poles on $\mathbb{C P}^{1}$ and let $\Gamma$ be a 1-cylinder separatrix diagram given by a pair of plane trees $\left(\mathcal{T}_{\text {bottom }}(\iota), \mathcal{T}_{\text {top }}(v-\iota)\right)$ with profiles $\iota$ and $v-\iota$, respectively; see Section 3.3. We have seen in Section 1.3 that any such separatrix diagram $\Gamma$ is realizable in $\mathcal{Q}\left(v,-1^{|v|+4}\right)$ and that any 1-cylinder separatrix diagram has this form.

Let $\mathcal{D}_{\mathbb{Z}, \Gamma, 1}^{\text {horiz }}$ be the set of square-tiled surfaces in $\mathcal{Q}\left(v,-1^{|v|+4}\right)$ having $\Gamma$ as the separatrix diagram and having a single horizontal band of squares. Recall that by convention, all zeros and poles of such square-tiled surfaces are labeled.

LEMMA 4.28. The number $\mathcal{N}_{\mathcal{D}_{\mathbb{Z}, \Gamma, 1}^{\text {horiz }}}\left(\mathcal{Q}\left(v,-1^{|v|+4}\right), 2 N\right)$ of all square-tiled surfaces sharing the fixed realizable 1-cylinder separatrix diagram $\Gamma$ and tiled with a single band of at most $2 N$ identical squares has the following asymptotics when $N \rightarrow+\infty$ :

$$
\mathcal{N}_{\mathcal{D}_{\mathbb{Z}, \Gamma, 1}^{\text {horiz }}}\left(\mathcal{Q}\left(v,-1^{|v|+4}\right), 2 N\right)=\operatorname{cyl}_{1}(\Gamma) \cdot \frac{N^{d}}{2 d}+o\left(N^{d}\right),
$$


where $d=\operatorname{dim}_{\mathbb{C}}\left(\mathcal{Q}\left(v,-1^{|v|+4}\right)\right)$ is given by equation (2.4) and

$$
\operatorname{cyl}_{1}(\Gamma)=\frac{4}{|\operatorname{Aut}(\Gamma)|} \cdot \frac{(|v|+4) ! \cdot v_{0} ! \cdot v_{1} ! \cdot v_{2} ! \cdots}{(|\iota|+\ell(\iota)) ! \cdot(|v-\iota|+\ell(v-\iota)) !} .
$$

Proof. In this section, we denote by $m$ the number of edges of $\mathcal{T}_{\text {bottom }}$ and we denote by $n$ the number of edges of $\mathcal{T}_{\text {top. }}$. The numbers $m$ and $n$ can be expressed as

$$
\begin{aligned}
m & =|\iota|+\ell(\iota)+1 \\
n & =|v-\iota|+\ell(v-\iota)+1
\end{aligned}
$$

and the dimension $d$ of the stratum satisfies relation $d=m+n$.

Consider any square-tiled surface having the diagram $\Gamma$ as the diagram of horizontal saddle connections. Cut it open along all horizontal saddle connections. By definition of $\Gamma$, it has $m$ pairs of saddle connections on one boundary component of the cylinder, $n$ pairs of saddle connections on the other boundary component of the cylinder and each saddle connection has its twin on the same boundary component.

The proof now follows line by line the second part of the proof of the more general [DGZZ, Proposition 2.2]. Note that the parameter $l$ used in Proposition 2.2 to denote the number of saddle connections which after the surgery as above appear on both sides of the cylinder is equal to zero in genus zero. One extra simplification comes from the fact that in the proof of [DGZZ, Proposition 2.2], we sum over various possible heights of the horizontal cylinder, while in our context, the height of the cylinder equals to the height of the square: the single horizontal cylinder of square-tiled surfaces in $\mathcal{D}_{\mathbb{Z}, \Gamma, 1}^{\text {horiz }}$ is tiled with a single band of squares. As a result, we do not get the extra factor $\zeta(d)$ present in the original expression (2.2) in [DGZZ, Proposition 2.2]; see equation (4.30) and the following remark.

REMARK 4.29. In this paper, we denote by $\operatorname{cyl}_{1}(\Gamma)$ the coefficient of the leading term in the asymptotics of the number of square-tiled surfaces sharing the fixed realizable 1-cylinder separatrix diagram $\Gamma$ and tiled with a single band of at most $2 N$ squares. Clearly, this number does not depend on the size of the identical squares. We tacitly assumed above that the squares are unit squares, but we could equally assume that our identical squares have size $\frac{1}{2} \times \frac{1}{2}$. The latter choice corresponds to our normalization for the Masur-Veech volume in Section 4.3.

Thus, $\operatorname{cyl}_{1}(\Gamma)=c\left(\mathcal{L}_{\mathbb{Z}, \Gamma, 1}^{\text {horiz }}\right)$ can be seen as the coefficient of the leading term in the asymptotics of the number of square-tiled surfaces tiled with at most $2 \mathrm{~N}$ squares of size $\frac{1}{2} \times \frac{1}{2}$, having a single horizontal cylinder of the minimal possible 
height $\frac{1}{2}$ and the separatrix diagram $\Gamma$. In the companion paper [DGZZ], we used a similar notation $c_{1}(\Gamma)$ for the coefficient in asymptotics where we made no restriction on the height of the cylinder. Choosing a different fixed height $h / 2$ of the cylinder, where $h \in \mathbb{N}$, one decreases the asymptotic number of square-tiled surfaces as above by the factor $h^{-d}$ (see the proof of [DGZZ, Proposition 2.2] for details). Here $d=\operatorname{dim}_{\mathbb{C}} \mathcal{Q}\left(v,-1^{|v|+4}\right)$ is given by formula (2.4). Moreover, the following summation formula holds:

$$
c_{1}(\Gamma)=c\left(\bigsqcup_{h \in \mathbb{N}} \mathcal{L}_{\mathbb{Z}, \Gamma, h}^{\text {horiz }}\right)=\sum_{h=1}^{+\infty} c\left(\mathcal{L}_{\mathbb{Z}, \Gamma, h}^{\text {horiz }}\right)=\zeta(d) \cdot \operatorname{cyl}_{1}(\Gamma) .
$$

This is a particular case of a general formula that holds for any number of cylinders in any arithmetic invariant orbifold. Compare also with the Remark 4.12.

4.10. Explicit count of square-tiled surfaces in genus 0 . In this section, we complete the Proof of Theorem 2.5 proving the remaining relation (2.7). We also prove Corollaries 4.30 and 4.31 used in the proofs of Theorems 1.1 and 1.2, respectively.

Consider a (generalized) partition $v=\left[0^{v_{0}} 1^{v_{1}} 2^{v_{2}} \ldots\right]$ of a natural number $|v|$ into the sum of nonnegative integer numbers (in this section, we allow entries 0 ):

$$
|v|:=\underbrace{0+\cdots+0}_{v_{0}}+\underbrace{1+\cdots+1}_{v_{1}}+\underbrace{2+\cdots+2}_{v_{2}}+\cdots .
$$

The common convention on Masur-Veech volumes of the strata of meromorphic quadratic differentials with at most simple poles suggests to label all zeros and poles. Following notations of Section 3.3, denote by $\mathcal{P}_{v}^{\text {labeled }}(N)$ the number of square-tiled surfaces with labeled zeros and poles in the stratum $\mathcal{Q}\left(v,-1^{|v|+4}\right)$ in genus zero tiled with at most $2 N$ identical squares and having a single horizontal and a single vertical band of squares. It is easy to see that a square-tiled surface as above cannot have any symmetries. Convention 3.3 on weights with which we count square-tiled surfaces with nonlabeled zeros and poles is designed to assure the following relation between the two counts valid for any $N \in \mathbb{N}$ :

$$
\mathcal{P}_{\nu}^{\text {labeled }}(N)=\left(\prod_{j=0}^{\infty} v_{j} !\right) \cdot(|v|+4) ! \cdot \mathcal{P}_{\nu}(N),
$$

where the product above contains, actually, only a finite number of factors. 
Recall that a type $\iota=\left[0^{\iota_{0}} 1^{\iota_{1}} 2^{\iota_{2}} \ldots\right]$ of a plane tree $\mathcal{T}$ records the number $\iota_{j}$ of vertices of valence $j+2$ for $j=0,1,2, \ldots$ Note that in Section 4.10, we allow the tree to have several vertices of valence 2 . Recall also that $|\nu|$ denotes the sum of the entries of the partition $v=\left[0^{\nu_{0}} 1^{v_{1}} 2^{\nu_{2}} \ldots\right]$; by $\ell(v)$, we denote the length of $v$, where, this time, we count the entries 0 if any

$$
\begin{aligned}
|v| & :=1 \cdot v_{1}+2 \cdot v_{2}+3 \cdot v_{3}+\cdots \\
\ell(v) & :=v_{0}+v_{1}+v_{2}+v_{3}+\cdots .
\end{aligned}
$$

Consider a separatrix diagram $\Gamma$ given by a pair of trees $\mathcal{T}_{\text {bottom }} \mathcal{T}_{\text {top }}$ as in Section 4.9. Defining the automorphism group $\operatorname{Aut}(\Gamma)$, we assume that none of the vertices, edges or boundary components of the ribbon graph $\Gamma$ is labeled; however, we assume that the orientation of the ribbons is fixed. Thus,

$$
|\operatorname{Aut}(\Gamma)|=\left|\operatorname{Aut}\left(\mathcal{T}_{\text {bottom }}\right)\right| \cdot\left|\operatorname{Aut}\left(\mathcal{T}_{\text {top }}\right)\right| \cdot \begin{cases}2 & \text { if } \mathcal{T}_{\text {bottom }} \simeq \mathcal{T}_{\text {top }} \\ 1 & \text { otherwise. }\end{cases}
$$

Here $\simeq$ stands for an isomorphism of plane ('ribbon') trees.

The following counting theorem for plane trees is well known; see, for example, [Mo, 2, p. 6]. It is the last element needed for the Proof of Theorem 2.5.

THEOREM. For any partition $\iota=\left[0^{\iota_{0}} 1^{\iota_{1}} 2^{\iota_{2}} \ldots\right]$, the following expression holds:

$$
\sum_{\mathcal{T} \text { with profile } \iota} \frac{1}{|\operatorname{Aut}(\mathcal{T})|}=\frac{(|\iota|+\ell(\iota)) !}{(|\iota|+2) ! \cdot \iota_{0} ! \cdot \iota_{1} ! \cdot \iota_{2} ! \cdots},
$$

where we sum over all plane trees corresponding to a partition ı and $|\operatorname{Aut}(\mathcal{T})|$ is the order of the automorphism group of the tree $\mathcal{T}$.

Now everything is ready to complete the Proof of Theorem 2.5.

Completion of the Proof of Theorem 2.5. It remains to prove expression (2.7).

Combining equation (4.29) with the above theorem, we conclude that the sum of $\operatorname{cyl}_{1}(\Gamma)$ over all realizable 1-cylinder separatrix diagrams $\Gamma$ in any given stratum $\mathcal{Q}\left(v,-1^{|v|+4}\right)$ in genus zero can be expressed as follows:

$$
\begin{aligned}
\operatorname{cyl}_{1}\left(\mathcal{Q}\left(v,-1^{|v|+4}\right)\right)=\sum_{\Gamma} \operatorname{cyl}_{1}(\Gamma) \\
=\frac{1}{2} \sum_{\iota \subset v}\left(\frac{4 \cdot(|v|+4) ! \cdot v_{0} ! \cdot v_{1} ! \cdot v_{2} ! \cdots}{(|\iota|+\ell(\iota)) ! \cdot(|v-\iota|+\ell(v-\iota)) !}\right)
\end{aligned}
$$




$$
\begin{aligned}
& \cdot\left(\frac{(|\iota|+\ell(\iota)) !}{(|\iota|+2) ! \cdot \iota_{0} ! \cdot \iota_{1} ! \cdots}\right) \cdot\left(\frac{(|v-\iota|+\ell(v-\iota)) !}{(|v-\iota|+2) ! \cdot\left(v_{0}-\iota_{0}\right) ! \cdot\left(v_{1}-\iota_{1}\right) ! \cdots}\right) \\
& =2 \sum_{\iota \subset v}\left(\begin{array}{l}
|v|+4 \\
|\iota|+2
\end{array}\right)\left(\begin{array}{l}
v_{0} \\
\iota_{0}
\end{array}\right)\left(\begin{array}{l}
v_{1} \\
\iota_{1}
\end{array}\right)\left(\begin{array}{l}
v_{2} \\
\iota_{2}
\end{array}\right) \cdots .
\end{aligned}
$$

We complete this section with two corollaries from Theorem 2.5.

COROLlaRY 4.30. For the partition $v=\left[1^{k}\right]$, the number $\mathcal{P}_{\left[1^{k}\right]}^{\text {labeled }}(N)$ of squaretiled surfaces with labeled zeros and poles in the stratum $\mathcal{Q}\left(1^{k},-1^{k+4}\right)$ tiled with at most $2 N$ identical squares and having a single horizontal and a single vertical band of squares, has the following asymptotics as $N \rightarrow+\infty$ :

$$
\mathcal{P}_{\left[1^{k}\right]}^{\text {labeled }}(N)=\operatorname{cyl}_{1,1}\left(\mathcal{Q}\left(1^{k},-1^{k+4}\right)\right) \cdot \frac{N^{2 k+2}}{4 k+4}+o\left(N^{2 k+2}\right) \text { as } N \rightarrow+\infty,
$$

where

$$
\operatorname{cyl}_{1,1}\left(\mathcal{Q}\left(1^{k},-1^{k+4}\right)\right)=\frac{\left(\operatorname{cyl}_{1}\left(\mathcal{Q}\left(1^{k},-1^{k+4}\right)\right)\right)^{2}}{4\left(\frac{\pi^{2}}{2}\right)^{k+1}}
$$

and

$$
\operatorname{cyl}_{1}\left(\mathcal{Q}\left(1^{k},-1^{k+4}\right)\right)=2 \cdot\left(\begin{array}{c}
2 k+4 \\
k+2
\end{array}\right) .
$$

The number $\mathcal{P}_{\left[0,1^{k}\right]}^{\text {labeled }}(N)$ of square-tiled surfaces as above with a single marked regular vertex of the tiling has the following asymptotics as $N \rightarrow+\infty$ :

$$
\mathcal{P}_{\left[0,1^{k}\right]}^{\text {labeled }}(N)=2 \cdot \operatorname{cyl}_{1,1}\left(\mathcal{Q}\left(1^{k},-1^{k+4}\right)\right) \cdot \frac{N^{2 k+3}}{4 k+6}+o\left(N^{2 k+3}\right) \text { as } N \rightarrow+\infty \text {. }
$$

Proof. By (2.2), we have

$$
\operatorname{Vol}_{1} \mathcal{Q}_{1}\left(1^{k},-1^{k+4}\right)=2 \pi^{2} \cdot\left(\frac{\pi^{2}}{2}\right)^{k}=4 \cdot\left(\frac{\pi^{2}}{2}\right)^{k+1} .
$$

To prove (4.33), we apply the following combinatorial identity to simplify formula (2.7) in the particular case when $v=\left[1^{k}\right]$ :

$$
\sum_{\iota_{1}=0}^{v_{1}}\left(\begin{array}{l}
v_{1} \\
\iota_{1}
\end{array}\right)\left(\begin{array}{c}
|v|+4 \\
|\iota|+2
\end{array}\right)=\sum_{\iota_{1}=0}^{k}\left(\begin{array}{l}
k \\
\iota_{1}
\end{array}\right)\left(\begin{array}{c}
k+4 \\
\iota_{1}+2
\end{array}\right)=\left(\begin{array}{c}
2 k+4 \\
k+2
\end{array}\right)
$$

see $[\mathbf{G d},(3.20)]$. 
It remains to prove that

$$
\operatorname{cyl}_{1,1}\left(\mathcal{Q}\left(1^{k}, 0,-1^{k+4}\right)\right)=2 \cdot \operatorname{cyl}_{1,1}\left(\mathcal{Q}\left(1^{k},-1^{k+4}\right)\right) .
$$

By (2.9), we have

$$
\operatorname{cyl}_{1,1}\left(\mathcal{Q}\left(1^{k}, 0,-1^{k+4}\right)\right)=\frac{\left(\operatorname{cyl}_{1}\left(\mathcal{Q}\left(1^{k}, 0,-1^{k+4}\right)\right)\right)^{2}}{\operatorname{Vol}_{1} \mathcal{Q}_{1}\left(1^{k}, 0,-1^{k+4}\right)} .
$$

Equation (2.7) implies that

$$
\operatorname{cyl}_{1}\left(\mathcal{Q}\left(1^{k}, 0,-1^{k+4}\right)\right)=2 \cdot \operatorname{cyl}_{1}\left(\mathcal{Q}\left(1^{k},-1^{k+4}\right)\right) .
$$

Finally, by (2.2), we have

$$
\operatorname{Vol}_{1} \mathcal{Q}_{1}\left(1^{k}, 0,-1^{k+4}\right)=2 \operatorname{Vol}_{1} \mathcal{Q}_{1}\left(1^{k},-1^{k+4}\right)
$$

and (4.34) follows.

We also prove the following elementary technical corollary of Theorem 2.5.

COROLlary 4.31. Consider a (generalized) partition $v=\left[0^{v_{0}} 1^{v_{1}} 2^{v_{2}} \ldots\right]$ and its subpartition $v^{\prime}=\left[1^{v_{1}} 2^{v_{2}} \ldots\right]$ obtained by suppressing all zero entries. The following formulas are valid:

$$
\begin{aligned}
\operatorname{cyl}_{1}\left(v,-1^{|v|+4}\right) & =2^{v_{0}} \cdot \operatorname{cyl}_{1}\left(v^{\prime},-1^{\left|v^{\prime}\right|+4}\right) \\
\mathrm{P}_{1}\left(v,-1^{|v|+4}\right) & =\mathrm{P}_{1}\left(v^{\prime},-1^{\left|\nu^{\prime}\right|+4}\right) .
\end{aligned}
$$

Proof. Note that $\left|v^{\prime}\right|=|v|$. Similarly, having any subpartition $\iota^{\prime} \subset \iota$ obtained from a partition $\iota$ by suppressing all zero entries, we have $\left|\iota^{\prime}\right|=|\iota|$. Thus, we can rewrite formula (2.7) as

$$
\begin{aligned}
& \operatorname{cyl}_{1}\left(\mathcal{Q}\left(\nu,-1^{|v|+4}\right)\right)=2 \cdot \sum_{\iota_{0}=0}^{\nu_{0}} \sum_{\iota_{1}=0}^{\nu_{1}} \sum_{\iota_{2}=0}^{\nu_{2}} \sum_{\ldots}^{\ldots}\left(\begin{array}{l}
\nu_{0} \\
\iota_{0}
\end{array}\right)\left(\begin{array}{l}
\nu_{1} \\
\iota_{1}
\end{array}\right)\left(\begin{array}{c}
\nu_{2} \\
\iota_{2}
\end{array}\right) \ldots\left(\begin{array}{l}
|v|+4 \\
|\iota|+2
\end{array}\right) \\
& =\left(\sum_{\iota_{0}=0}^{v_{0}}\left(\begin{array}{l}
v_{0} \\
\iota_{0}
\end{array}\right)\right) \cdot\left(2 \sum_{\iota_{1}=0}^{v_{1}} \sum_{\iota_{2}=0}^{v_{2}} \ldots\left(\begin{array}{l}
v_{1} \\
\iota_{1}
\end{array}\right) \cdots\left(\begin{array}{c}
\left|v^{\prime}\right|+4 \\
\left|\iota^{\prime}\right|+2
\end{array}\right)\right) \\
& =2^{\nu_{0}} \operatorname{cyl}_{1}\left(\mathcal{Q}\left(v^{\prime},-1^{\left|v^{\prime}\right|+4}\right)\right) \text {, }
\end{aligned}
$$

which proves (4.35). To prove (4.36), it suffices to note that by formula (2.2), we have

$\operatorname{Vol}_{1} \mathcal{Q}_{1}\left(v,-1^{|v|+4}\right)=(f(0))^{\nu_{0}} \operatorname{Vol}_{1} \mathcal{Q}_{1}\left(v^{\prime},-1^{\left|v^{\prime}\right|+4}\right)=2^{v_{0}} \operatorname{Vol}_{1} \mathcal{Q}_{1}\left(v^{\prime},-1^{\left|v^{\prime}\right|+4}\right)$. 
Passing to the ratios

$$
\begin{aligned}
\mathrm{P}_{1}\left(\mathcal{Q}\left(v,-1^{|v|+4}\right)\right): & =\frac{\operatorname{cyl}_{1}\left(\mathcal{Q}\left(v,-1^{|v|+4}\right)\right)}{\operatorname{Vol}_{1}\left(\mathcal{Q}_{1}\left(v,-1^{|v|+4}\right)\right)} \\
& =\frac{\operatorname{cyl}_{1}\left(\mathcal{Q}\left(v^{\prime},-1^{\left|v^{\prime}\right|+4}\right)\right)}{\operatorname{Vol}_{1}\left(\mathcal{Q}_{1}\left(v^{\prime},-1^{\left|v^{\prime}\right|+4}\right)\right)}=: \mathrm{P}_{1}\left(\mathcal{Q}\left(v^{\prime},-1^{\left|v^{\prime}\right|+4}\right)\right),
\end{aligned}
$$

we get the desired equation (4.35).

\section{Acknowledgements}

The vague idea that counting results concerning linear involutions might have applications to meanders was discussed by the authors in independent conversations with M. Kontsevich and with Mirzakhani. We are grateful to them for these discussions and for their insights in enumerative geometry that were very inspiring for us.

We thank MPIM in Bonn, where part of this work was performed, for providing us with friendly and stimulating environment. We thank J. Athreya and the anonymous referee for helpful suggestions that allowed to improve the presentation. We are grateful to A. Eskin for the important advice on Moore's ergodicity theorem. We thank E. Duriev for reading carefully the manuscript and for useful comments.

Research of Section 3 was conducted at Saint Petersburg State University under support of the RSF grant 19-71-30002.

\section{Conflict of Interest: None.}

\section{Appendix A. Lattices in strata of meromorphic quadratic differentials and associated square-tiled surfaces}

The definition of the Masur-Veech volume of strata of meromorphic quadratic differentials with at most simple poles involves several normalization conventions. The mismatch in the choice of one of the conventions is a constant source of confusion. We describe in Section A.1 various conventions and specify the one used in the current paper. We discuss the topological origin of the lattices giving rise to different normalizations of the volume element and describe the square-tiled surfaces associated with these lattices.

Throughout this Appendix, by a stratum $\mathcal{Q}\left(v,-1^{|v|+4}\right)$ of meromorphic quadratic differentials in genus zero, we always mean the stratum in the moduli 
space of meromorphic quadratic differentials $\left(\mathbb{C P}^{1}, q\right)$ such that $q$ has $v_{0}$ marked points, $v_{i}$ zeros of order $i$ for $i=1,2, \ldots$ and $|\nu|+4$ simple poles and no other poles, where $|v|=v_{1}+2 v_{2}+\cdots$. Since we use only strata in genus zero in the study of meanders, we limit our considerations in this Appendix to genus zero where certain normalization issues do not manifest. Note that all strata in genus zero are nonempty and connected.

A.1. Lattices in strata of meromorphic quadratic differentials. By convention, all zeros and simple poles of Abelian or quadratic differentials are always numbered (labeled). Thus, expressing Masur-Veech volumes through the count of square-tiled surfaces, one has to either label conical singularities on square-tiled surfaces (which is uncommon) or apply necessary normalization by the product of factorials as in (4.31).

Next, there are two particularly natural ways to define the integer lattice in period coordinates $H_{-}^{1}(\hat{X}, \hat{\Sigma} ; \mathbb{C})$ (the period coordinates for the strata of quadratic differentials were defined at the end of Section 4.1).

One can either choose as the 'integer lattice' the following set:

$$
\text { \{elements of } \left.H_{-}^{1}(\hat{X}, \hat{\Sigma} ; \mathbb{C}) \text { taking values in } \mathbb{Z} \oplus i \mathbb{Z} \text { on } H_{1}^{-}(\hat{X}, \hat{\Sigma} ; \mathbb{Z})\right\}
$$

or, alternatively, one can choose as the 'integer lattice' the set defined as follows:

$$
H_{-}^{1}(\hat{X}, \hat{\Sigma} ; \mathbb{C}) \cap H^{1}(\hat{X}, \hat{\Sigma} ; \mathbb{Z} \oplus i \mathbb{Z}) .
$$

The difference between the two choices reveals itself in the linear holonomy along saddle connections joining two distinct zeros. Under the first convention, the linear holonomy along such saddle connections belongs to the half-integer lattice $\frac{1}{2} \mathbb{Z} \oplus \frac{i}{2} \mathbb{Z}$, while under the second convention, it belongs to the integer lattice $\mathbb{Z} \oplus i \mathbb{Z}$.

Actually, the set $\hat{\Sigma}$ admits alternative natural definitions; the choice of one of them is a matter of another convention. In the construction of the canonical double cover, the preimages of the simple poles of $q$ on $X$ become regular points of $\omega$ on the canonical double cover $\hat{X}$. One can either choose to mark the resulting regular points and consider them as part of the data of the cover or not. In other words, one has to make a choice whether to include these points in $\hat{\Sigma}$ or not. We reserve notation $\hat{\Sigma}$ for the set where all these marked points are included, and we use notation $\hat{\Sigma}^{\prime}$ for the set which does not contain preimages of simple poles. In genus zero, when simple poles are always present, $\hat{\Sigma}^{\prime}$ is always a proper subset of $\hat{\Sigma}$. For example, depending on this choice, the stratum $\mathcal{Q}\left(1^{2},-1^{6}\right)$ is realized as an invariant arithmetic suborbifold either in $\mathcal{H}\left(0^{6}, 2^{2}\right)$ or in $\mathcal{H}\left(2^{2}\right)$. 
Consider now the following lattices:

$$
\begin{aligned}
\mathbb{L} & :=H_{-}^{1}(\hat{X}, \hat{\Sigma} ; \mathbb{C}) \cap H^{1}(\hat{X}, \hat{\Sigma} ; \mathbb{Z} \oplus i \mathbb{Z}), \\
\mathbb{L}^{\prime} & :=H_{-}^{1}\left(\hat{X}, \hat{\Sigma}^{\prime} ; \mathbb{C}\right) \cap H^{1}\left(\hat{X}, \hat{\Sigma}^{\prime} ; \mathbb{Z} \oplus i \mathbb{Z}\right), \\
\frac{1}{2} \mathbb{L} & :=H_{-}^{1}(\hat{X}, \hat{\Sigma} ; \mathbb{C}) \cap H^{1}\left(\hat{X}, \hat{\Sigma} ; \frac{1}{2} \mathbb{Z} \oplus \frac{i}{2} \mathbb{Z}\right)
\end{aligned}
$$

LEMMA A.1. Consider a stratum $\mathcal{Q}\left(v,-1^{|v|+4}\right)$ of meromorphic quadratic differentials in genus zero. The natural map

$$
H_{-}^{1}(\hat{X}, \hat{\Sigma} ; \mathbb{C}) \rightarrow H_{-}^{1}\left(\hat{X}, \hat{\Sigma}^{\prime} ; \mathbb{C}\right)
$$

induced by the inclusion $\hat{\Sigma}^{\prime} \subset \hat{\Sigma}$ is an isomorphism of vector spaces.

Proof. We start by constructing a convenient set of cycles, which, depending on interpretation, provides a basis in both $H_{1}^{-}(\hat{X}, \hat{\Sigma} ; \mathbb{Z})$ and $H_{1}^{-}\left(\hat{X}, \hat{\Sigma}^{\prime} ; \mathbb{Z}\right)$. This will prove, in particular, that (A.2) is an isomorphism.

Consider the following oriented non-self-intersecting path $\rho$ on the sphere $X=\mathbb{C P}^{1}$ at the base of the cover $\hat{X} \rightarrow X$. The path $\rho$ starts at a zero or at a marked point of $\Sigma$, then it passes through all other zeros and marked points of $\Sigma$, and only then $\rho$ passes through all simple poles but one. We consider $\rho$ as a curvilinear broken line with vertices in $\Sigma$. For every oriented segment $\gamma_{j}=[P, Q]$ of this broken line (where $P, Q$ are two points of $\Sigma$ passed by $\rho$ consecutively), consider the two preimages $\gamma_{j}^{\prime}, \gamma_{j}^{\prime \prime}$ of $\gamma$ on $\hat{X}$ endowed with the orientation induced from $\gamma_{j}$ and consider the cycle $\left[\hat{\gamma}_{j}\right]:=\left[\gamma_{j}^{\prime}\right]-\left[\gamma_{j}^{\prime \prime}\right] \in H_{1}^{-}(\hat{X}$, $\hat{\Sigma} ; \mathbb{Z})$. It is easy to see that the resulting collections of cycles forms a basis in $H_{1}^{-}(\hat{X}, \hat{\Sigma} ; \mathbb{Z})$.

By assumption, $X$ has genus zero, and quadratic differential $q$ has only simple poles, so $\Sigma$ always contains at least four poles of $q$. Note that we intentionally omitted one pole in our construction. Extending the broken line to the remaining pole and completing our collection of cycles with the resulting extra cycle $\hat{\gamma}_{0}=\left[\gamma_{0}^{\prime}\right]-\left[\gamma_{0}^{\prime \prime}\right]$ in $H_{1}^{-}(\hat{X}, \hat{\Sigma} ; \mathbb{Z})$, we would get a collection of cycles satisfying a linear relation: the sum of the cycles (taken with appropriate signs) corresponding to all segments of the extended broken line is zero in $H_{1}^{-}(\hat{X}$, $\hat{\Sigma} ; \mathbb{Z})$.

We explain now why the constructed collection of paths provides a basis in $H_{1}^{-}\left(\hat{X}, \hat{\Sigma}^{\prime} ; \mathbb{C}\right)$ as well. Note that all simple poles are branch points of the cover. Recall that $\rho$ visits first all zeros and marked points of $\Sigma$ and only then passes through simple poles. Thus, all cycles $\left[\hat{\gamma}_{j}\right]:=\left[\gamma_{j}^{\prime}\right]-\left[\gamma_{j}^{\prime \prime}\right]$, for $j=1, \ldots, \ell(\nu)-1$, are well-defined cycles in $H_{1}^{-}\left(\hat{X}, \hat{\Sigma}^{\prime} ; \mathbb{Z}\right)$. 
Let $\gamma_{\ell(v)}=[P, Q]$ be the segment joining the last zero (or a marked point) $P \in \Sigma$ to the first simple pole $Q \in \Sigma$. Consider that path $\hat{\gamma}_{\ell(v)}:=\gamma_{\ell(v)}^{\prime}-\gamma_{\ell(v)}^{\prime \prime}$ on $\hat{X}$ which first follows $\gamma_{\ell(v)}^{\prime}$ and then, when it arrives at the preimage of the simple pole $Q$, it follows $\gamma_{\ell(v)}^{\prime \prime}$ in the opposite direction. We get a connected path $\hat{\gamma}_{\ell(v)}$ which is closed if $P$ is a zero of odd degree. The path $\hat{\gamma}_{\ell(v)}$ is a segment with endpoints at two preimages $P^{\prime}, P^{\prime \prime}$ of $P$ when $P$ is a regular point of the cover $p: \hat{X} \rightarrow X$ (zero of even degree of $q$ or a marked point). Note that in the latter case, both $P^{\prime}$ and $P^{\prime \prime}$ belong to $\hat{\Sigma}^{\prime}$, so in both cases, $\hat{\gamma}_{\ell(v)}$ is a well-defined cycle in $H_{1}^{-}\left(\hat{X}, \hat{\Sigma}^{\prime} ; \mathbb{Z}\right)$. Similarly, any segment $\gamma=\left[Q_{j}, Q_{j+1}\right]$ of $\rho$ joining two simple poles defines a closed cycle $[\hat{\gamma}]=\left[\gamma^{\prime}-\gamma^{\prime \prime}\right]$ in $H_{1}^{-}\left(\hat{X}, \hat{\Sigma}^{\prime} ; \mathbb{Z}\right)$. It is immediate to see that the resulting cycles also form a basis but, this time, already in $H_{1}^{-}\left(\hat{X}, \hat{\Sigma}^{\prime} ; \mathbb{Z}\right)$. This proves, in particular, that the natural map (A.2) induced by the inclusion $\hat{\Sigma}^{\prime} \subset \hat{\Sigma}$ is an isomorphism of vector spaces.

LEMMA A.2. Consider a stratum $\mathcal{Q}\left(v,-1^{|v|+4}\right)$ of meromorphic quadratic differentials in genus zero. The lattice $\frac{1}{2} \mathbb{L}$ coincides with the lattice defined by (A.1)

$\frac{1}{2} \mathbb{L}=\left\{\right.$ elements of $H_{-}^{1}(\hat{X}, \hat{\Sigma} ; \mathbb{C})$ taking values in $\mathbb{Z} \oplus i \mathbb{Z}$ on $\left.H_{1}^{-}(\hat{X}, \hat{\Sigma} ; \mathbb{Z})\right\}$.

Proof. Consider the basis of cocycles $\alpha_{1}, \ldots, \alpha_{d}$ in $H_{-}^{1}(\hat{X}, \hat{\Sigma} ; \mathbb{C})$ dual to the basis of cycles $\hat{\gamma}_{i}$ in $H_{1}^{-}(\hat{X}, \hat{\Sigma} ; \mathbb{Z})$ constructed in the Proof of Lemma A.1. By definition of a dual basis, we have $\alpha_{i}\left(\hat{\gamma}_{j}\right)=\delta_{i, j}$. This implies that the collection $\alpha_{1}, \ldots, \alpha_{d}, i \alpha_{1}, \ldots, i \alpha_{d}$ serves as a basis of the lattice (A.1). Let us show now that $\alpha_{1}, \ldots, \alpha_{d}, i \alpha_{1}, \ldots, i \alpha_{d}$ is also a basis of the lattice $\frac{1}{2} \mathbb{L}$.

Denote by $\tau$ the canonical involution of $\hat{X}$ associated with the ramified double cover $p: \hat{X} \rightarrow X$. For any $c \in H_{1}(\hat{X}, \hat{\Sigma} ; \mathbb{C})$ and for any $\theta \in H_{-}^{1}(\hat{X}, \hat{\Sigma} ; \mathbb{C})$, we have

$$
\theta\left(\tau_{*}(c)\right)=\left(\tau^{*} \theta\right)(c)=-\theta(c)
$$

since, by definition, the subspace $H_{-}^{1}(\hat{X}, \hat{\Sigma} ; \mathbb{C})$ is anti-invariant with respect to the involution $\tau^{*}$. By construction, $\hat{\gamma}_{j}=\left[\gamma_{j}^{\prime}\right]-\left[\gamma_{j}^{\prime \prime}\right]=\left[\gamma_{j}^{\prime}\right]-\tau_{*}\left[\gamma_{j}^{\prime}\right]$. Thus, by (A.3),

$$
\alpha_{i}\left(\left[\hat{\gamma}_{j}^{\prime}\right]\right)=-\alpha_{i}\left(\left[\hat{\gamma}_{j}^{\prime \prime}\right]\right)=\frac{1}{2} \delta_{i, j}
$$

for any $i, j \in\{1, \ldots, d\}$. Since $\sum_{j=0}^{d}\left[\hat{\gamma}_{j}\right]=0$, we conclude that

$$
\alpha_{i}\left(\left[\hat{\gamma}_{0}^{\prime}\right]\right)=-\alpha_{i}\left(\left[\hat{\gamma}_{0}^{\prime \prime}\right]\right) \in \frac{1}{2} \mathbb{Z} \quad \text { for } i=1, \ldots, d .
$$

The relative cycles $\left[\hat{\gamma}_{0}^{\prime}\right],\left[\hat{\gamma}_{0}^{\prime \prime}\right],\left[\hat{\gamma}_{1}^{\prime}\right],\left[\hat{\gamma}_{1}^{\prime \prime}\right], \ldots,\left[\hat{\gamma}_{d}^{\prime}\right],\left[\hat{\gamma}_{d}^{\prime \prime}\right]$ generate $H_{1}(\hat{X}, \hat{\Sigma} ; \mathbb{Z})$. We conclude that all basic cocycles $\alpha_{j}$ and $i \alpha_{j}$, where $j=1, \ldots, d$, of the 
lattice (A.1) in $H_{-}^{1}(\hat{X}, \hat{\Sigma} ; \mathbb{C})$ take values in $\frac{1}{2} \mathbb{Z} \oplus \frac{i}{2} \mathbb{Z}$ on $H_{1}(\hat{X}, \hat{\Sigma} ; \mathbb{Z})$, which proves the inclusion

$$
\frac{1}{2} \mathbb{L} \supseteq\left\{\text { elements of } H_{-}^{1}(\hat{X}, \hat{\Sigma} ; \mathbb{C}) \text { taking values in } \mathbb{Z} \oplus i \mathbb{Z} \text { on } H_{1}^{-}(\hat{X}, \hat{\Sigma} ; \mathbb{Z})\right\} \text {. }
$$

A similar consideration proves the inclusion in the other direction.

The following lemma shows that the lattice (A.1) is independent of the choice of $\hat{\Sigma}$ or $\hat{\Sigma}^{\prime}$.

LEMMA A.3. Consider a stratum $\mathcal{Q}\left(v,-1^{|v|+4}\right)$ of meromorphic quadratic differentials in genus zero. The convention on the choice of $\hat{\Sigma}$ or $\hat{\Sigma}^{\prime}$ does not affect the lattice (A.1): the discrete subsets of $H_{-}^{1}(\hat{X}, \hat{\Sigma} ; \mathbb{C})$ and of $H_{-}^{1}(\hat{X}$, $\left.\hat{\Sigma}^{\prime} ; \mathbb{C}\right)$ defined by $(A .1)$ are in bijective correspondence under the natural map $H_{-}^{1}(\hat{X}, \hat{\Sigma} ; \mathbb{C}) \rightarrow H_{-}^{1}\left(\hat{X}, \hat{\Sigma}^{\prime} ; \mathbb{C}\right)$.

Proof. By Lemma A.1, the natural linear map (A.2) induced by the inclusion $\hat{\Sigma}^{\prime} \subset \hat{\Sigma}$ is an isomorphism of vector spaces. Consider a basis of cocycles in each of these spaces dual to the basis of integer cycles $\hat{\gamma}_{j}, j=1, \ldots, d$, in $H_{1}^{-}(\hat{X}$, $\hat{\Sigma} ; \mathbb{Z})$ and in $H_{1}^{-}\left(\hat{X}, \hat{\Sigma}^{\prime} ; \mathbb{Z}\right)$ respectively, constructed in the Proof of Lemma A.1. The lattices induced by these bases are exactly the discrete subsets of $H_{-}^{1}(\hat{X}$, $\hat{\Sigma} ; \mathbb{C})$ and of $H_{-}^{1}\left(\hat{X}, \hat{\Sigma}^{\prime} ; \mathbb{C}\right)$ defined by (A.1). Moreover, it follows from the construction of the two bases that the isomorphism (A.2) sends one to the other.

Now we are ready to prove inclusions (A.4) of lattices defined above and compute the resulting indices of sublattices.

LEMMA A.4. Consider a stratum $\mathcal{Q}\left(v,-1^{|v|+4}\right)$ of meromorphic quadratic differentials in genus zero. The isomorphism (A.2) induces the following chain of inclusions of lattices:

$$
\mathbb{L} \subset \mathbb{L}^{\prime} \subset \frac{1}{2} \mathbb{L}
$$

The indices of these sublattices satisfy the following relations:

$$
\begin{aligned}
\left|\frac{1}{2} \mathbb{L}: \mathbb{L}^{\prime}\right| & =4^{\ell(v)-1} \\
\left|\mathbb{L}^{\prime}: \mathbb{L}\right| & =4^{|v|+3} .
\end{aligned}
$$

REMARK A.5. Note that the lattice $\frac{1}{2} \mathbb{L}$ can be obtained from the lattice $\mathbb{L}$ by subdividing the mesh by the factor of 2 or, equivalently, by applying homothety 
with coefficient $\frac{1}{2}$ to the lattice $\mathbb{L}$. Thus, the index $\left|\frac{1}{2} \mathbb{L}: \mathbb{L}\right|$ equals $2^{2 d}=4^{d}$, where $d=\operatorname{dim}_{\mathbb{C}} \mathcal{Q}\left(v,-1^{|v|+4}\right)=\ell(v)+|v|+2$ so that

$$
\left|\frac{1}{2} \mathbb{L}: \mathbb{L}\right|=4^{\ell(v)+|v|+2} .
$$

The lemma above computes the two remaining indices.

Proof of Lemma A.4. The fact that the map (A.2) is a linear isomorphism of vector spaces allows us to consider the lattices $\mathbb{L}$ and $\frac{1}{2} \mathbb{L}$ as sublattices of $H_{-}^{1}(\hat{X}$, $\left.\hat{\Sigma}^{\prime} ; \mathbb{C}\right)$ and allows to consider the lattice $\mathbb{L}^{\prime}$ as a sublattice of $H_{-}^{1}(\hat{X}, \hat{\Sigma} ; \mathbb{C})$.

Passing from the bases of $H_{1}^{-}(\hat{X}, \hat{\Sigma} ; \mathbb{Z})$ and $H_{1}^{-}\left(\hat{X}, \hat{\Sigma}^{\prime} ; \mathbb{Z}\right)$ to the dual bases in $H_{-}^{1}(\hat{X}, \hat{\Sigma} ; \mathbb{Z})$ and in $H_{-}^{1}\left(\hat{X}, \hat{\Sigma}^{\prime} ; \mathbb{Z}\right)$, respectively, we use the resulting cohomology classes to construct bases of the corresponding lattices. In this way, we prove the inclusions (A.4). It remains to compute the indices of these sublattices in the ambient lattices.

Let $\gamma_{1}, \ldots, \gamma_{d}$ be the consecutive segments of the path $\rho$ constructed in the Proof of Lemma A.1. Here

$$
d=\operatorname{dim}_{\mathbb{C}} H_{-}^{1}(\hat{X}, \hat{\Sigma} ; \mathbb{C})=\operatorname{dim}_{\mathbb{C}} H_{-}^{1}\left(\hat{X}, \hat{\Sigma}^{\prime} ; \mathbb{C}\right)=\ell(v)+|v|+2 .
$$

By construction, the first $\ell(\nu)-1$ segments of $\rho$ have the endpoints at zeros and at marked points of $\Sigma$, while the remaining $|v|+3$ segments have the endpoints at $|v|+3$ (that is, all but one) simple poles.

Passing from the lattice $\frac{1}{2} \mathbb{L}$ to its sublattice $\mathbb{L}^{\prime}$, we impose the extra condition that the value of the cocycles in the sublattice on each individual relative cycle $\left[\gamma_{j}^{\prime}\right],\left[\gamma_{j}^{\prime \prime}\right]$ belongs to $\mathbb{Z} \oplus i \mathbb{Z}$ for $j=1, \ldots, \ell(\nu)-1$ (that is, for those cycles which involve points from $\hat{\Sigma}^{\prime}$ ). The cocycles in the sublattice $\frac{1}{2} \mathbb{L}$ take values in $\frac{1}{2} \mathbb{Z} \oplus \frac{i}{2} \mathbb{Z}$ on these cocycles. Note that since these cocycles belong to the subspace $H_{-}^{1}(\hat{X}, \hat{\Sigma} ; \mathbb{C})$ anti-invariant with respect to the hyperelliptic involution, their values on all pairs of symmetric cycles $\left[\gamma_{j}^{\prime}\right]$ and $\left[\gamma_{j}^{\prime \prime}\right]$ are coherent: both real and imaginary parts are simultaneously integer or half-integer. This gives the index $\left|\frac{1}{2} \mathbb{L}: \mathbb{L}^{\prime}\right|=2^{2(\ell(v)-1)}$ as claimed in (A.5). Extra factor 2 in the exponent of 2 comes from the fact that we have to take into account both real and imaginary parts.

To prove (A.6), one can either combine (A.7) and (A.5) or notice directly that passing from the lattice $\mathbb{L}^{\prime}$ to its sublattice $\mathbb{L}$, we impose the extra condition that the value of the cocycles in the sublattice $\mathbb{L}$ on each individual relative cycle $\left[\gamma_{j}^{\prime}\right],\left[\gamma_{j}^{\prime \prime}\right]$ belongs to $\mathbb{Z} \oplus i \mathbb{Z}$ for $j=\ell(\nu), \ldots, \ell(v)+|v|+2$, while the cocycles in the ambient lattice $\mathbb{L}^{\prime}$ evaluated on these cycles take values in $\frac{1}{2} \mathbb{Z} \oplus \frac{i}{2} \mathbb{Z}$.

Consider the Masur-Veech volume elements on a stratum $\mathcal{Q}\left(v,-1^{|v|+4}\right)$ of meromorphic quadratic differentials in genus zero corresponding to the above 
lattices. We have proved in Lemma A.1 that we can use any of the two isomorphic vector spaces

$$
H_{-}^{1}(\hat{X}, \hat{\Sigma} ; \mathbb{C}) \simeq H_{-}^{1}\left(\hat{X}, \hat{\Sigma}^{\prime} ; \mathbb{C}\right)
$$

as period coordinates in $\mathcal{Q}\left(v,-1^{|v|+4}\right)$. Recall that there is a natural oneparameter family of volume elements in any finite-dimensional vector space $V$; any two volume elements in this family differ by a constant factor. Any lattice of maximal rank in $V$ determines the distinguished normalization of the volume element by the condition that the volume of the fundamental domain of the lattice is equal to 1. Considering one of the vector spaces in (A.8) and one of the three lattices (A.4), we get three different normalizations of the Masur-Veech volume element on $\mathcal{Q}\left(v,-1^{|v|+4}\right)$. Denote by $\operatorname{Vol}_{1}^{\frac{1}{2} \mathbb{L}} \mathcal{Q}_{1}\left(v,-1^{|v|+4}\right), \operatorname{Vol}_{1}^{\mathbb{L}^{\prime}} \mathcal{Q}_{1}\left(v,-1^{|v|+4}\right)$ and $\operatorname{Vol}_{1}^{\mathbb{L}} \mathcal{Q}_{1}\left(v,-1^{|v|+4}\right)$ the volumes of the stratum $\mathcal{Q}\left(v,-1^{|v|+4}\right)$ with respect to these volume elements.

COROLlary A.6. The Masur-Veech volumes of a stratum $\mathcal{Q}\left(v,-1^{|v|+4}\right)$ of meromorphic differentials in genus zero induced by the lattices (A.4) satisfy the following relations:

$$
\begin{aligned}
\operatorname{Vol}_{1}^{\frac{1}{2} \mathbb{L}} \mathcal{Q}_{1}\left(v,-1^{|v|+4}\right): \operatorname{Vol}_{1}^{\mathbb{L}^{\prime}} \mathcal{Q}_{1}\left(v,-1^{|v|+4}\right) & =4^{\ell(v)-1}, \\
\operatorname{Vol}_{1}^{\mathbb{L}^{\prime}} \mathcal{Q}_{1}\left(\nu,-1^{|v|+4}\right): \operatorname{Vol}_{1}^{\mathbb{L}} \mathcal{Q}_{1}\left(v,-1^{|v|+4}\right) & =4^{|v|+3} .
\end{aligned}
$$

Proof. The Masur-Veech volume element is defined as linear volume element in period coordinates normalized by an appropriate lattice. Hence, passing from a lattice to a sublattice, we change the normalization of the Masur-Veech measure by the constant factor equal to the index of the sublattice.

\section{A.2. Degrees of correspondences induced by canonical double covers.} The comparison of lattices in the previous section was performed in period coordinates, that is, locally. This local computation allowed us to keep track of the change of the Masur-Veech volume $\operatorname{Vol}_{1} \mathcal{Q}_{1}\left(v,-1^{|v|+4}\right)$ when passing from one lattice normalization to the other. Up to now, we always stayed on $\mathcal{Q}(v$, $\left.-1^{|v|+4}\right)$.

Recall that the canonical ramified double cover construction associates to every stratum of meromorphic quadratic differentials with at most simple poles an invariant arithmetic suborbifold $\mathcal{L}$ in the corresponding stratum of Abelian differentials, where the preimages of all simple poles are marked; see Section 4.3. The invariant arithmetic suborbifold $\mathcal{L}$ is endowed with the natural cover $P$ : $\mathcal{L} \rightarrow \mathcal{Q}\left(v,-1^{|v|+4}\right)$. 
Alternatively, we can apply the double cover construction without marking the preimages of simple poles. In this way, we obtain an alternative invariant arithmetic orbifold $\mathcal{L}^{\prime}$ in a different stratum of Abelian differentials. For example, under the first construction, we associate to the stratum $\mathcal{Q}\left(1^{2},-1^{6}\right)$ an invariant arithmetic suborbifold $\mathcal{L}$ in $\mathcal{H}\left(2^{2}, 0^{6}\right)$, while under the second construction, we associate to the same stratum $\mathcal{Q}\left(1^{2},-1^{6}\right)$ an invariant arithmetic suborbifold $\mathcal{L}^{\prime}$ in $\mathcal{H}\left(2^{2}\right)$. The resulting invariant arithmetic orbifolds $\mathcal{L}$ and $\mathcal{L}^{\prime}$ are related by the natural forgetful map $F: \mathcal{L} \rightarrow \mathcal{L}^{\prime}$ forgetting the preimages of the simple poles. The choice between $\mathcal{L}$ and $\mathcal{L}^{\prime}$ corresponds to the choice between the subsets $\hat{\Sigma}$ or $\hat{\Sigma}^{\prime}$ in the local computations of Section A.1.

Recall that we have a canonical Definition (4.5) of the Masur-Veech volume of any arithmetic invariant orbifold. In this section, we compare the Masur-Veech volumes $\operatorname{Vol}_{1} \mathcal{Q}_{1}\left(v,-1^{|v|+4}\right), \operatorname{Vol}_{1} \mathcal{L}_{1}$ and $\operatorname{Vol}_{1} \mathcal{L}_{1}^{\prime}$.

There is a natural correspondence between $\mathcal{Q}\left(v,-1^{|v|+4}\right)$ and $\mathcal{L}$. Each zero of odd order of $q$ and each simple pole of $q$ has a single preimage on $\hat{X}$. However, each zero of even order of $q$ and each marked point has two distinct preimages and both preimages are zeros (respectively marked points) of $\omega$. We denote by

$$
\ell\left(v_{\text {even }}\right):=v_{0}+v_{2}+v_{4}+\cdots
$$

the total number of marked points and of zeros of even orders. Note also that the triples $(\hat{X}, \omega, \hat{\Sigma})$ and $(\hat{X},-\omega, \hat{\Sigma})$ represent the same point of the stratum of Abelian differentials with unlabeled zeros. These considerations show that we have $2^{\ell\left(v_{\text {even }}\right)}$ ways of labeling the preimages of zeros of even degrees. A priori, the resulting set $\widehat{\mathcal{Q}}\left(v,-1^{|v|+4}\right)$ of labeled Abelian differentials may be not connected. By definition, $\mathcal{L}$ denotes any of the isomorphic connected components of $\widehat{\mathcal{Q}}\left(\nu,-1^{|v|+4}\right)$.

LEMMA A.7. Consider a stratum $\mathcal{Q}\left(v,-1^{|v|+4}\right)$ of meromorphic quadratic differentials in genus zero. The subset $\widehat{\mathcal{Q}}\left(v,-1^{|v|+4}\right)$ of Abelian differentials obtained by applying the canonical double cover construction to all $(X, q)$ in $\mathcal{Q}\left(\nu,-1^{|v|+4}\right)$ with marked preimages of simple poles is connected.

Proof. The projectivization $\mathrm{P} \mathcal{Q}\left(v,-1^{|v|+4}\right)=\mathcal{Q}\left(v,-1^{|v|+4}\right) / \mathbb{C}^{*}$ of any stratum $\mathcal{Q}\left(v,-1^{|v|+4}\right)$ in genus zero is isomorphic to the space of configurations of $\ell(v)+|v|+4$ distinct labeled points on $\mathbb{C P}^{1}$ (the locations of the zeros, of the marked points and of the simple poles of the quadratic differential $q$ ). In particular, given any zero of $q$ of even order (where we consider the marked points as zeros of $q$ of order zero), one can always construct a closed trajectory in $\mathcal{Q}\left(v,-1^{|v|+4}\right)$ by moving this zero close to a simple pole, then around this pole and then backtracking the zero to the initial position following backwards the 
path which we used to move it close to the simple pole. Lifting the resulting loop in $\mathcal{Q}\left(v,-1^{|v|+4}\right)$ to a path in $\widehat{\mathcal{Q}}\left(v,-1^{|v|+4}\right)$, we exchange the labels of the two preimages of this zero of even order. This construction implies connectedness of $\widehat{\mathcal{Q}}\left(\nu,-1^{|v|+4}\right)$.

Considering the correspondence between $\mathcal{Q}\left(v,-1^{|v|+4}\right)$ and $\mathcal{L}^{\prime}$, we observe the following additional phenomenon. The set $\hat{\Sigma}$ can be reconstructed from $\hat{\Sigma}^{\prime}$ as an unlabeled set by adding all ramification points of $\hat{X}$ which are not yet in $\hat{\Sigma}^{\prime}$. However, since the preimages of the simple poles are not marked in $\hat{\Sigma}^{\prime}$ and thus not labeled, the information on the labels of simple poles in $(X, q, \Sigma)$ is lost when passing to the canonical double cover $\left(\hat{X}, \omega, \hat{\Sigma}^{\prime}\right)$. Thus, the points of $\mathcal{Q}(v$, $\left.-1^{|v|+4}\right)$ and the points of $\mathcal{L}^{\prime}$ are in the natural $(|\nu|+4) !: 2^{\ell\left(v_{\text {even }}\right)}$ correspondence, where $|v|+4$ is the number of simple poles.

Let $p: \hat{X} \rightarrow X$ be the canonical ramified double cover such that $p^{*} q=\omega^{2}$. Note that the flat area defined by the Abelian differential $\omega$ on $\hat{X}$ is twice the area defined by the initial quadratic differential $q$ on $X$. Thus, under the correspondence of the stratum $\mathcal{Q}\left(v,-1^{|v|+4}\right)$ of quadratic differentials and the suborbifold in the associated stratum of Abelian differentials, the hypersurface $\mathcal{L}_{1}$ corresponds to the subset of quadratic differentials $(X, q)$ of flat area $\frac{1}{2}$. Following the tradition, we denote this locus by $\mathcal{Q}_{1}\left(\nu,-1^{|v|+4}\right)$, that is,

$$
\mathcal{Q}_{1}\left(v,-1^{|v|+4}\right):=\left\{(X, q) \in \mathcal{Q}\left(v,-1^{|v|+4}\right) \mid \operatorname{Area}(X, q)=\frac{1}{2}\right\} .
$$

Given a Masur-Veech volume element $d \operatorname{Vol}$ on $\mathcal{Q}\left(\nu,-1^{|v|+4}\right)$, we define

$$
\operatorname{Vol}_{1} \mathcal{Q}_{1}\left(v,-1^{|v|+4}\right):=2 d \cdot \operatorname{Vol} C_{1} \mathcal{Q}_{1}\left(\nu,-1^{|v|+4}\right),
$$

where the cone $C_{1} \mathcal{Q}_{1}\left(v,-1^{|v|+4}\right)$ is defined as

$$
C_{1} \mathcal{Q}_{1}\left(v,-1^{|v|+4}\right):=\left\{(X, q) \in \mathcal{Q}\left(v,-1^{|v|+4}\right) \mid \operatorname{Area}(X, q) \leqslant \frac{1}{2}\right\} .
$$

The numerical values in (2.2) were obtained using this definition and the MasurVeech volume element on $\mathcal{Q}\left(v,-1^{|v|+4}\right)$ normalized by the lattice (A.1); by Lemma A.2, this lattice coincides with the lattice $\frac{1}{2} \mathbb{L}$.

Consider the locus $\mathcal{L}^{\prime}$ of Abelian differentials consisting of all canonical double covers of all $(X, q)$ in $\mathcal{Q}\left(v,-1^{|v|+4}\right)$, where the preimages of simple poles are not marked. Let $P: \mathcal{L} \rightarrow \mathcal{Q}\left(v,-1^{|v|+4}\right)$ be the natural cover and let $F: \mathcal{L} \rightarrow \mathcal{L}^{\prime}$ be the forgetful map (forgetting the marked points that are preimages of poles). We complete this section with the following lemma summarizing the above considerations.

Lemma A.8. Consider any normalization $d \mathrm{Vol}^{*}$ of the Masur-Veech volume element on a stratum $\mathcal{Q}\left(v,-1^{|v|+4}\right)$ of meromorphic quadratic differentials in 
genus zero and consider the volume element on $\mathcal{L}$ induced by $d \mathrm{Vol}^{*}$ via the cover $P: \mathcal{L} \rightarrow \mathcal{Q}\left(v,-1^{|v|+4}\right)$. The resulting volume element on $\mathcal{L}$ can be induced from a volume element $d \mathrm{Vol}^{*}$ on $\mathcal{L}^{\prime}$ by means of the forgetful map $F: \mathcal{L} \rightarrow \mathcal{L}^{\prime}$. The volumes $\operatorname{Vol}_{1}^{*} \mathcal{L}_{1}, \operatorname{Vol}_{1}^{*} \mathcal{L}_{1}^{\prime}$ and $\operatorname{Vol}_{1}^{*} \mathcal{Q}_{1}\left(v,-1^{|v|+4}\right)$ satisfy the following relations:

$$
\begin{aligned}
& \operatorname{Vol}_{1}^{*} \mathcal{L}_{1}:=2^{\ell\left(v_{\text {even }}\right)} \cdot \operatorname{Vol}_{1}^{*} \mathcal{Q}_{1}\left(v,-1^{|v|+4}\right) \\
& \operatorname{Vol}_{1}^{*} \mathcal{L}_{1}^{\prime}:=\frac{2^{\ell\left(v_{\text {even }}\right)}}{(|v|+4) !} \cdot \operatorname{Vol}_{1}^{*} \mathcal{Q}_{1}\left(\nu,-1^{|v|+4}\right) .
\end{aligned}
$$

Proof. The proof is essentially reduced to the observation that $\operatorname{deg}(P)=2^{\ell\left(v_{\text {even }}\right)}$ and that $\operatorname{deg}(F)=(|v|+4)$ !.

A.3. Lattice points as square-tiled surfaces. In this section, we discuss the square tiling in the flat metric associated with lattice points $(X, q, \Sigma),(\hat{X}, \omega, \hat{\Sigma})$, $\left(\hat{X}, \omega, \hat{\Sigma}^{\prime}\right)$ for the lattices $\frac{1}{2} \mathbb{L}, \mathbb{L}$ and $\mathbb{L}^{\prime}$, respectively.

We have seen that lattice points in period coordinates of an invariant arithmetic suborbifold represent square-tiled surfaces. Speaking of a square tiling, we always assume that all the squares of the tiling are identical and polarized, that is, we know which pair of opposite sides of each square is horizontal; the remaining pair of sides is vertical. Gluing the squares, we identify sides to sides respecting the polarization. Constructing a square-tiled surface representing an Abelian differential, we impose additional translation structure.

We always consider only those square tilings of $(X, q, \Sigma)$ (respectively of $(\hat{X}$, $\left.q, \hat{\Sigma}),\left(\hat{X}, q, \hat{\Sigma}^{\prime}\right)\right)$ for which all the points of the discrete subset $\Sigma$ (respectively of $\left.\hat{\Sigma}, \hat{\Sigma}^{\prime}\right)$ are located at vertices of the squares.

LEMMA A.9. Consider a stratum $\mathcal{Q}\left(v,-1^{|v|+4}\right)$ of meromorphic quadratic differentials in genus zero.

(1) $\frac{1}{2} \mathbb{L}$-lattice points in $\mathcal{Q}\left(v,-1^{|v|+4}\right)$ are those triples $(X, q, \Sigma)$ for which the induced (polarized) flat metric admits square tiling by $\frac{1}{2} \times \frac{1}{2}$ squares. The induced normalization $d \mathrm{Vol}^{\frac{1}{2} \mathbb{L}}$ of the Masur-Veech volume element on $\mathcal{Q}(v$, $\left.-1^{|v|+4}\right)$ combined with definitions (A.9) and (A.10) provides the same value of the Masur-Veech volume $\operatorname{Vol}_{1} \mathcal{Q}_{1}\left(v,-1^{|v|+4}\right)$ as (4.6).

(2) $\mathbb{L}$-lattice points in $\mathcal{Q}\left(v,-1^{|v|+4}\right)$ are those triples $(X, q, \Sigma)$ for which the induced (polarized) flat metric admits square tiling by unit squares. The induced Masur-Veech volume element $d \mathrm{Vol}^{\mathbb{L}}$ on $\mathcal{Q}\left(v,-1^{|v|+4}\right)$ satisfies

$$
d \mathrm{Vol}^{\mathbb{L}}=4^{d} \cdot d \mathrm{Vol}^{\frac{1}{2} \mathbb{L}}
$$


(3) $\mathbb{L}$-lattice points in the arithmetic invariant suborbifold $\mathcal{L}$ are those triples $(\hat{X}, \omega, \hat{\Sigma})$ for which the induced (polarized) flat metric admits square tiling by unit squares. The associated Masur-Veech volume element $d \mathrm{Vol}^{\mathbb{L}}$ on $\mathcal{L}$ coincides with the volume element induced from the volume element $d \mathrm{Vol}^{\mathbb{L}}$ on $\mathcal{Q}\left(v,-1^{|v|+4}\right)$ by means of the cover $P: \mathcal{L} \rightarrow \mathcal{Q}\left(v,-1^{|v|+4}\right)$. It provides the same value of the Masur-Veech volume $\mathrm{Vol}_{1} \mathcal{L}_{1}$ as (4.5).

(4) $\mathbb{L}$ '-lattice points in the arithmetic invariant suborbifold $\mathcal{L}^{\prime}$ are those triples $\left(\hat{X}, \omega, \hat{\Sigma}^{\prime}\right)$ for which the induced (polarized) flat metric admits square tiling by unit squares. The induced normalization $d \mathrm{Vol}^{\mathbb{L}^{\prime}}$ of the Masur-Veech volume element on $\mathcal{L}^{\prime}$ provides the same value of the Masur-Veech volume $\mathrm{Vol}_{1} \mathcal{L}_{1}^{\prime}$ as (4.5).

Proof. By Lemma A.2, the lattices $\frac{1}{2} \mathbb{L}$ and (A.1) coincide. Upon this observation, the assertion (1) becomes a direct corollary of Lemma B.1 from [AEZ2] (reproduced as Lemma A.11).

Statement (2) is proved in Remark A.5.

The assertions of the remaining statements (3) and (4) concerning the square tilings are proved by the following standard argument; see, for example, [Zor2]. We recall the proof for the lattice $\mathbb{L}$; the proof for $\mathbb{L}^{\prime}$ is completely analogous.

By definition of the lattice $\mathbb{L}$, the relative cohomology class $[\omega]$ of any $\mathbb{L}$ lattice point $(\hat{X}, \omega, \hat{\Sigma})$ in $\mathcal{L}$ belongs to $H^{1}(\hat{X}, \hat{\Sigma} ; \mathbb{Z} \oplus i \mathbb{Z})$. Fix a point $P \in \hat{\Sigma}$. Consider the map $p_{\omega}: X \rightarrow \mathbb{C} /(\mathbb{Z} \oplus i \mathbb{Z})$ defined as

$$
p_{\omega}: Q \mapsto\left(\int_{P}^{Q} \omega\right) \bmod \mathbb{Z} \oplus i \mathbb{Z}
$$

The condition $[\omega] \in H^{1}(\hat{X}, \hat{\Sigma} ; \mathbb{Z} \oplus i \mathbb{Z})$ implies that the map does not depend on the path joining the distinguished point $P$ to a point $Q$ of $X$, so $p_{\omega}$ is well defined. It is easy to see that $p_{\omega}$ is a ramified cover and all ramification points of $p_{\omega}$ belong to $\hat{\Sigma}$. Consider the flat torus $\mathbb{C} /(\mathbb{Z} \oplus i \mathbb{Z})$ as a unit square with the identified opposite sides. The cover $p_{\omega}$ endows $X$ with a tiling by unit squares. By construction, all points from $\hat{\Sigma}$ are located in the corners of the resulting square tiling and, thus, the assertions of statements (3) and (4) concerning the square tilings are proved.

The coincidence of the volume element $d \mathrm{Vol}^{\mathbb{L}}$ on $\mathcal{L}$ with the volume element obtained by pulling back the volume element $d \mathrm{Vol}^{\mathbb{L}}$ on $\mathcal{Q}\left(v,-1^{|v|+4}\right)$ by means of the cover $P: \mathcal{L} \rightarrow \mathcal{Q}\left(v,-1^{|v|+4}\right)$ follows from the fact that both $\mathcal{L}$ and $\mathcal{Q}(v$, $\left.-1^{|v|+4}\right)$ share the same local period coordinates $H_{-}^{1}(\hat{X}, \hat{\Sigma}, \mathbb{C})$ in which the two volume elements are determined by the same lattice $\mathbb{L}$. The cover $P$ acts in these coordinates as the identity map. 
Finally, the assertions about the values of the resulting Masur-Veech volumes $\operatorname{Vol}_{1} \mathcal{L}_{1}$ and $\operatorname{Vol}_{1} \mathcal{L}_{1}^{\prime}$ in (3) and (4), respectively, now follow from equivalence of Definition (4.4) (where we, respectively, take $\mathcal{L}_{1}$ or $\mathcal{L}_{1}^{\prime}$ as $V_{1}$ ) and Definition (4.5); this equivalence was proved in Section 4.2.

We are now ready to prove Proposition 4.3. Though, formally speaking, we provide a Proof of Proposition 4.3 only for strata of meromorphic quadratic differentials in genus zero, the general case is completely analogous with the exception for connectedness of $\widehat{\mathcal{Q}}(\xi)^{\text {comp }}$ which is claimed in Proposition 4.3 only for strata in genus zero.

Proof of Proposition 4.3. Let $(\hat{X}, \omega, \hat{\Sigma})$ be a square-tiled surface in $\mathcal{L}$. By construction, the cover $p_{\omega}: \hat{X} \rightarrow \mathbb{T}$ constructed in the Proof of Lemma A.9 intertwines the involutions $\tau: \hat{X} \rightarrow \hat{X}$ and $\iota: \mathbb{T} \rightarrow \mathbb{T}$, where $\iota$ is the elliptic involution on $\mathbb{T}$ :

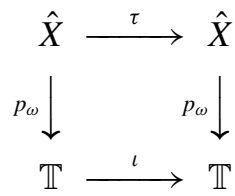

This implies that the involution $\tau$ maps the squares of the tiling onto squares of the tiling.

The involution $\tau$ maps $\omega$ to $-\omega$, that is, $\tau^{*} \omega=-\omega$. Thus, if one of the squares maps to itself, this is done by the central symmetry, which fixes the center of the square. Hence, the center of this square would be the preimage of a simple pole of $q$ on $X$ which contradicts the assumption that the points of $\hat{\Sigma}$ are located only at the corners of the squares. This argument proves assertions (1) and (2) of Proposition 4.3.

Assertion (3) of Proposition 4.3 follows directly from assertions (2) and (3) of Lemma A.9.

The remaining assertion (4) of Proposition 4.3 is specific for genus zero. It was already proved in Lemma A.7.

Throughout Section 4, we have predominantly considered the invariant arithmetic orbifold $\mathcal{L}$. Corollary A.10 describes the important particular case when it is natural to consider the invariant arithmetic orbifold $\mathcal{L}^{\prime}$.

COROllary A.10. Consider a stratum $\mathcal{Q}\left(k,-1^{k+4}\right)$ of those meromorphic quadratic differentials in genus zero, which have a single zero of order $k$, where $k \in \mathbb{N}$, and $k+4$ simple poles. The associated invariant arithmetic orbifold $\mathcal{L}^{\prime}$ in the corresponding stratum of Abelian differentials coincides with the 
hyperelliptic connected component $\mathcal{H}^{\text {hyp }}(k+1)$ when $k$ is odd and with the hyperelliptic connected component $\mathcal{H}^{\text {hyp }}\left(\left(\frac{k}{2}\right)^{2}\right)$ when $k$ is even.

In this particular case, the lattices $\frac{1}{2} \mathbb{L}$ and $\mathbb{L}^{\prime}$ coincide and thus the MasurVeech volumes $\operatorname{Vol}_{1} \mathcal{L}_{1}^{\prime}$ and $\operatorname{Vol}_{1} \mathcal{Q}_{1}\left(k,-1^{k+4}\right)$ defined by (4.5) and (4.6), respectively, are related by (A.12).

Proof. The first assertion is the definition of a hyperelliptic connected component; see [KoZo].

The coincidence of lattices $\frac{1}{2} \mathbb{L}$ and $\mathbb{L}^{\prime}$ follows from (A.5). Namely, in our case, the partition $v$ contains a single entry, and, thus, $4^{\ell(v)-1}=1$. Hence, choosing in Lemma A.8 the Masur-Veech volume element $d \operatorname{Vol}^{\frac{1}{2} \mathbb{L}}$ on $\mathcal{Q}\left(k,-1^{k+4}\right)$, we get as the induced volume element $d \mathrm{Vol}^{\frac{1}{2} \mathbb{L}}$ on $\mathcal{L}^{\prime}$ the Masur-Veech volume element $d \mathrm{Vol}^{\mathbb{L}^{\prime}}$. Thus, the corresponding volumes are related by formula (A.12).

It remains to note that by Lemma A.9, the Masur-Veech volume $\operatorname{Vol}_{1} \mathcal{L}_{1}^{\prime}$ defined by (4.5) corresponds to the normalization of the Masur-Veech volume element on $\mathcal{L}^{\prime}$ by the lattice $\mathbb{L}^{\prime}$ and that the Masur-Veech volume $\operatorname{Vol}_{1} \mathcal{Q}_{1}(k$, $-1^{k+4}$ ) defined by (4.6) corresponds to the normalization of the Masur-Veech volume element on $\mathcal{Q}\left(k,-1^{k+4}\right)$ by the lattice $\frac{1}{2} \mathbb{L}$ or, equivalently, by the lattice (A.1).

Remark 1.2 at the end of [AEZ2, Section 1.1] presents the following two illustrations of Corollary A.10 (see (2.2) for the definition of $f$ ):

$$
\begin{aligned}
\operatorname{Vol}_{1} \mathcal{Q}_{1}\left(1,-1^{5}\right) & =2 \pi^{2} f(1)(f(-1))^{5} \\
& =2 \pi^{2} \cdot \frac{\pi^{2}}{2} \cdot 1^{5}=5 ! \cdot \frac{\pi^{4}}{120}=5 ! \cdot \operatorname{Vol}_{1} \mathcal{H}_{1}(2), \\
\operatorname{Vol}_{1} \mathcal{Q}_{1}\left(2,-1^{6}\right) & =2 \pi^{2} f(2)(f(-1))^{6}=2 \pi^{2} \cdot \frac{4 \pi^{2}}{3} \cdot 1^{6} \\
& =\frac{6 !}{2} \cdot \frac{\pi^{4}}{135}=\frac{6 !}{2} \cdot \operatorname{Vol}_{1} \mathcal{H}_{1}\left(1^{2}\right) .
\end{aligned}
$$

We complete this Appendix reproducing the statement of the result from [AEZ2, Appendix B], proving that in genus zero, the $\frac{1}{2}$-square-tiled surfaces $(X, q, \Sigma)$ (that is, the points of the stratum corresponding to the lattice $\frac{1}{2} \mathbb{L}$ or, equivalently, to the same lattice defined as (A.1)) are represented by pillowcase covers specified below. We warn the reader that the statement of Lemma A.11 is not valid for genera higher than zero.

Let $\Lambda \subset \mathbb{C}$ be a lattice, and let $\mathbb{T}^{2}=\mathbb{C} / \Lambda$ be the associated torus. The quotient

$$
\mathcal{P}:=\mathbb{T}^{2} / \pm
$$


by the map $z \rightarrow-z$ is known as the pillowcase orbifold. It is a sphere with four $(\mathbb{Z} / 2)$-orbifold points (the corners of the pillowcase). The quadratic differential $(d z)^{2}$ on $\mathbb{T}^{2}$ descends to a quadratic differential on $\mathcal{P}$. Viewed as a quadratic differential on the Riemann sphere, $(d z)^{2}$ has simple poles at corner points. When the lattice $\Lambda$ is the standard integer lattice $\mathbb{Z} \oplus i \mathbb{Z}$, the flat torus $\mathbb{T}^{2}$ is obtained by isometrically identifying the opposite sides of a unit square, and the pillowcase $\mathcal{P}$ is obtained by isometrically identifying two $\frac{1}{2} \times \frac{1}{2}$ squares along the perimeter; see the right picture in Figure 4 in Section 2.1.

Consider a connected ramified cover $\hat{\mathcal{P}}$ over $\mathcal{P}$ of degree $d$ having ramification points only over the corners of the pillowcase. Clearly, $\hat{\mathcal{P}}$ is tiled by $2 d$ squares of the size $\frac{1}{2} \times \frac{1}{2}$. Coloring the two squares of the pillowcase $\mathcal{P}$ one in black and the other in white, we get a chessboard coloring of the square tiling of the cover $\hat{\mathcal{P}}$ : the white squares are always glued to the black ones and vice versa.

LEMmA A.11 [AEZ2]. Let $S=(X, q, \Sigma)$ be a point in the stratum $\mathcal{Q}(v$, $\left.-1^{|v|+4}\right)$ of meromorphic quadratic differentials in genus zero. The following properties are equivalent:

(1) $S$ represents a point of the lattice $(A .1)$ in $\mathcal{Q}\left(v,-1^{|v|+4}\right)$;

(2) $S$ is a cover over $\mathcal{P}$ ramified only over the corners of the pillowcase;

(3) $S$ is tiled by black and white $\frac{1}{2} \times \frac{1}{2}$ squares in the chessboard order.

Lemma A.11 implies that any square-tiled surface in any stratum of meromorphic quadratic differentials in genus zero is always tiled with even number of squares and that such tiling always admits chessboard coloring.

Note that in genera one and higher, one finds square-tiled surfaces tiled with odd number of squares. In genera one and higher, one also finds square-tiled surfaces tiled with an even number of squares which, nevertheless, do not admit chessboard coloring. Note also that 'pillowcase covers' are defined differently by different authors; see, for example, [EOk].

\section{References}

[ACPRS] J. E. Andersen, L. O. Chekhov, R. C. Penner, C. M. Reidys and P. Sułkowski, 'Topological recursion for chord diagrams, RNA complexes, and cells in moduli spaces', Nuclear Phys. B 866(3) (2013), 414-443.

[Arn] V. I. Arnold, 'Ramified covering $\mathbb{C P}^{2} \rightarrow S^{4}$, hyperbolicity and projective topology', Sib. Math. J. 29(5) (1988), 36-47.

[AEZ1] J. Athreya, A. Eskin and A. Zorich, 'Counting generalized Jenkins-Strebel differentials', Geom. Dedicata 170(1) (2014), 195-217. 
[AEZ2] J. Athreya, A. Eskin and A. Zorich, 'Right-angled billiards and volumes of moduli spaces of quadratic differentials on $\mathbb{C P}^{1}$, Ann. Sci. Éc. Norm. Supér. (4) 49 (2016), 1307-1381.

[DGZZ] V. Delecroix, E. Goujard, P. Zograf and A. Zorich, 'Contribution of one-cylinder square-tiled surfaces to Masur-Veech volumes', Astérisque 415(1) (2020), with an Appendix by P. Engel, to appear.

[DGZZ2] V. Delecroix, E. Goujard, P. Zograf and A. Zorich, 'Square-tiled surfaces with fixed combinatorics in invariant arithmetic suborbifolds: density, equidistribution, noncorrelation', in preparation.

[DGZZ3] V. Delecroix, E. Goujard, P. Zograf and A. Zorich, 'Masur-Veech volumes, frequencies of simple closed geodesics and intersection numbers of moduli spaces of curves', Preprint, 2019, arXiv:1908.08611.

[DiFGG1] P. Di Francesco, O. Golinelli and E. Guitter, 'Meander, folding, and arch statistics', Math. Comput. Model. 26(8-10) (1997), 97-147.

[DiFGG2] P. Di Francesco, O. Golinelli and E. Guitter, 'Meanders: exact asymptotics', Nuclear Phys. B 570(3) (2000), 699-712.

[DdHb] A. Douady and J. Hubbard, 'On the density of Strebel differentials', Invent. Math. 30(2) (1975), 175-179.

[EHL] D. El-Baz, B. Huang and M. Lee, 'Effective equidistribution of primitive rational points on expanding horospheres', Preprint, 2018, arXiv:1811.04019.

[EMi] A. Eskin and M. Mirzakhani, 'Invariant and stationary measures for the $\mathrm{SL}_{2}(\mathbb{R})$ action on moduli space', Publ. Inst. Hautes Etudes Sci. 127 (2018), 95-324.

[EMiMo1] A. Eskin, M. Mirzakhani and A. Mohammadi, 'Isolation, equidistribution, and orbit closures for the $\mathrm{SL}_{2}(\mathbb{R})$-action on moduli space', Ann. of Math. (2) 182(2) (2015), 673-721.

[EMiMo2] A. Eskin, M. Mirzakhani and A. Mohammadi, 'Effective counting of simple closed geodesics on hyperbolic surfaces', Preprint 2019, arXiv:1905.04435.

[EOk] A. Eskin and A. Okounkov, 'Pillowcases and quasimodular forms', in Algebraic Geometry and Number Theory, Honor of Vladimir Drinfeld's 50th Birthday, (ed.

G. Victor) Progress in Mathematics, 253 (Birkhäuser, Basel, 2006), 1-25.

[G1] E. Goujard, 'Siegel-Veech constants for strata of moduli spaces of quadratic differentials', GAFA 25(5) (2015), 1440-1492.

[G2] E. Goujard, 'Volumes of strata of moduli spaces of quadratic differentials: getting explicit values', Ann. Inst. Fourier (Grenoble) 66(6) (2016), 2203-2251.

[Gd] H. W. Gould, 'Combinatorial identities. A standardized set of tables listing 500 binomial coefficient summations' Rev. ed. (English) Morgantown (1972).

[Jen] I. Jensen, 'A transfer matrix approach to the enumeration of plane meanders', J. Phys. A 33(34) (2000), 5953-5963.

[KoZo] M. Kontsevich and A. Zorich, 'Connected components of the moduli spaces of Abelian differentials with prescribed singularities', Invent. Math. 153(3) (2003), 631-678.

[LdZv] S. K. Lando and A. K. Zvonkin, 'Plane and projective meanders', Theoret. Comput. Sci. 117 (1993), 227-241.

[LNW] E. Lanneau, D.-M. Nguyen and A. Wright, 'Finiteness of Teichmüller curves in nonarithmetic rank 1 orbit closures', Amer. J. Math. 139(6) (2017), 1449-1463.

[Mas] H. Masur, 'Interval exchange transformations and measured foliations', Ann. of Math. (2) 115 (1982), 169-200. 
[Mi] M. Mirzakhani, 'Growth of the number of simple closed geodesics on hyperbolic surfaces', Ann. of Math. (2) 168(1) (2008), 97-125.

[MiWri] M. Mirzakhani and A. Wright, 'The boundary of an affine invariant submanifold', Invent. Math. 209(3) (2017), 927-984.

[Mo] J. W. Moon, Counting Labelled Trees, Canadian Mathematical Monographs, 1 (Canadian Mathematical Congress, Montreal, 1970), 113.

[NRW] A. Nevo, R. Rühr and B. Weiss, 'Effective counting on translation surfaces', $A d v$. Math. 360 (2020), 106890.

[Po] H. Poincaré, 'Sur un téorème de géométrie', Rend. Circ. Mat. Palermo (2) 33 (1912), 375-407. (Oeuvres, T.VI, 499-538).

[Str] K. Strebel, Quadratic Differentials, Ergebnisse der Mathematik und ihrer Grenzgebiete (Springer, Berlin, 1984), 184.

[Vee1] W. Veech, 'Gauss measures for transformations on the space of interval exchange maps', Ann. of Math. (2) 115 (1982), 201-242.

[Wri1] A. Wright, 'The field of definition of affine invariant submanifolds of the moduli space of Abelian differentials', Geom. Topol. 18(3) (2014), 1323-1341.

[Wri2] A. Wright, 'Cylinder deformations in orbit closures of translation surfaces', Geom. Topol. 19(1) (2015), 413-438.

[Zim] R. J. Zimmer, Ergodic Theory and Semisimple Groups, Monographs in Mathematics, 81 (Birkhäuser, Boston-Basel-Stuttgart, 1984), 209.

[Zor1] A. Zorich, 'Flat surfaces', in Frontiers in Number Theory, Physics, and Geometry. I (Springer, Berlin, 2006), 437-583.

[Zor2] A. Zorich, 'Square-tiled surfaces and Teichmüller volumes of the moduli spaces of Abelian differentials', in Rigidity in Dynamics and Geometry (Springer, Berlin, 2002), 459-471, (Cambridge, 2000).

[Zor3] A. Zorich, 'Explicit Jenkins-Strebel representatives of all strata of Abelian and quadratic differentials', J. Mod. Dyn. 2(1) (2008), 139-185. 